\title{
Redox two-component initiated free radical and cationic polymerizations: Concepts, reactions and applications
}

\author{
Patxi Garra ${ }^{\mathrm{a}, \mathrm{b}}{ }_{*}$, Céline Dietlin ${ }^{\mathrm{a}, \mathrm{b}}$, Fabrice Morlet-Savary ${ }^{\mathrm{a}, \mathrm{b}}$, Frédéric Dumur ${ }^{\mathrm{c}}$, Didier Gigmes ${ }^{\mathrm{c}}$, \\ Jean-Pierre Fouassier ${ }^{\text {a, }}$, Jacques Lalevée ${ }^{\mathrm{a}, \mathrm{b}, *}$ \\ a Université de Haute-Alsace, CNRS, IS2M UMR 7361, F-68100, Mulhouse, France \\ ${ }^{\mathrm{b}}$ Université de Strasbourg, France \\ c Aix-Marseille Univ, CNRS, ICR, F-13397, Marseille, France
}

\section{A R T I C L E I N F O}

Article history:

Received 27 September 2018

Received in revised form 24 April 2019

Accepted 30 April 2019

Available online xxx

Keywords:

Redox polymerization

Reducing agents

Oxidizing agents

Redox initiating systems RIS

Controlled polymerization

Composites

\begin{abstract}
A B S T R A C T
Redox polymerizations are of huge importance throughout academic and industrial polymer science. Many authors propose the reaction of reducing (Red) and oxidizing (Ox) agents to accelerate/initiate radical or cationic polymerizations. As a result of activation energies typically below $80 \mathrm{~kJ} / \mathrm{mol}$, such reactions can occur under mild conditions, e.g., at room temperature, with reduced energy consumptions and robust in applications, such as the fabrication of composites. However, a clear definition of redox polymerization can only be found in reviews dealing with redox free radical polymerizations (FRP) publis ren wenty years ago (or more). Therefore, a fresh and broader update is provided here for more recent wor when the "Red" and "Ox" agents constituting the redox initiating system are mixed under mild conditions are presented, followed by a discussion of the redox FRP initiating systems in bulk. Initiating systems dealing with the redox cationic polymerization (CP) are reviewed, and parc lels between conventional FRP/CP and controlled polymerizations, in which redox systems are used, $\mathrm{i}$ vided. Many red $\mathrm{X}$ a rents are useful in both modes. Finally, dual-cure (redox/photochemical; redox dual FRP/CP) systems is, ented and selected applications are reviewed. Altogether, the state of the art for redox two-component polymerizations is provided, along with some perspectives.
\end{abstract}

\section{Introduction: Redox polymerization concepts}

Chain-growth free radical polymerizations (FRP) and cationic polymerizations (CP) are of major importance in the polymer industry. For example, more than $45 \%$ of polymer production is through FRP processes [1]. Polymer synthetic's conditions lead to outstand-

Abbreviations: ARGET, activators regenerated by electron transfer; ATRP, atom transfer radical polymerization; BDE, bond dissociation energy; BPO, dibenzoyl peroxide; CP, cationic polymerization; CRP, controlled/living radical polymerization; EPOX, (3,4-epoxycyclohexane)methyl 3,4-epoxycyclohexylcarboxylate; FRP, free radical polymerization; FRPCP, free radical promoted cationic photopolymerization; FTIR, Fourier transform infrared spectroscopy; Iod, diphenyliodoniumhexafluorophosphate; IPN, interpenetrated polymer network; LED, light-emitting diode; NIR, near infrared; NVK, N-vinylcarbazole; PIS, photoinitiating systems; RAFT, reversible addition-fragmentation chain transfer; RCP, redox cationic polymerization; RFRP, redox free radical photopolymerization; RIS, redox initiating systems; RT, room temperature; SCE, Saturated calomel electrode; TMPTA, trimethylolpropanetriacrylate; UV, ultra-violet

* Corresponding authors at: Université de Haute-Alsace, CNRS, IS2M UMR 7361, F-68100, Mulhouse, France.

Email addresses: patxi.garra@gmail.com (P. Garra); jacques.lalevee@uha.fr (J. Lalevée)

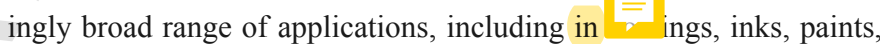
adhesives, composites, latex production, packing industry, biomaterials, etc. The current trend and one of the challenges is to produce polymers under limited energy consumption. Here, we focus on polymerization initiation through a two components mixing approach where a redox reaction enables the formation of active species such as radicals or cations. Redox initiating strategies are of outmost interest as they facilitate fast production of functional polymers at room temperature (RT), generally und and without any additional energetic consumption [2]. In add al, no further purification of the concerned monomers is necessary when efficient redox initiating systems (RIS) are used. Compared to photopolymerization, mostly applied to coatings and/or thin layer polymerization [3], redox initiated polymerizations show extremely high robustness in reaction media where light penetration is limited and/or absent (composites/thick samples/dispersions/...) [4-6].

Redox polymerization occurs when a reducing agent (or system) is mixed with an oxidizing agent (or system) generating reactive species that allow the polymerization of the surrounding monomers (or oligomers, Scheme 1) [7,8]. Examples in the literature are mainly (>95\%) appliad to FRP. Redox initiating systems were fortuitously discovered in 19,7 by German researchers who found an accelerating effect of reducing agents in thermal radical initiating systems [7,9-13]. The search for efficient redox initiating systems was a ma- 


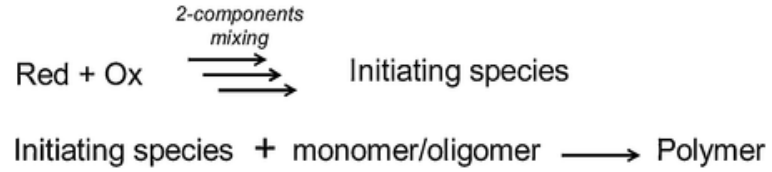

Scheme 1. Redox initiated polymerization from the two components mixing of reducing (Red) and oxidizing (Ox) systems.

jor component among the strategies used to initiate polymerization from 1945 to the end of the 90's [7,8,14,15]. Typically, oxidizing agents show low bond dissociation energies (BDE), e.g. peroxides or other compounds bearing weak $\mathrm{O}-\mathrm{O}$ or $\mathrm{S}-\mathrm{S}$ bonds) resulting in low activation energies when used in combination with reducing agents (typically $40-80 \mathrm{~kJ} / \mathrm{mol}$ ), which allows reactions to occur under mild conditions (room or even lower temperature) compared to thermal polymerization. The latter concerns initiating strategies for which heating of the reaction media leads to the thermolysis of compounds bearing low BDE chemical bonds (120-170 kJ/mol).

\subsection{Two-component mixing: solids, liquids, emulsions, and surfaces}

Many processes may be developed for redox initiation (Scheme 2); the essence being that a reducing system (Red) is mixed with an oxidizing system $(\mathrm{Ox})$ ding to the production of initiating species. A few possibilities of $\ldots$ Red and Ox speciation phases are summarized in Scheme 2. The reducing and oxidizing agents can be solubilized in the monomers (sometimes in the presence of solvent) in the plest approach; if polymerizations proceed in a dispersed media $\overline{ }$ edox species may also be preferentially encountered in the micelle (or the solvent) phase. Solubilization of a solid oxidizing agent (e.g. ammonium persulfate) in a monomer (such as acrylic acid) may be used in the

starting for redox initiation. In that case, solubilization kinetics must produce radicals fast enough to counter the dynamic polymerization termination processes (e.g. the oxygen inhibition in RFRP). Redox polymer grafting to (or from) surfaces containing Red or Ox may be applied under mild conditions [16-19]. Popular eynmyles of $\mathrm{Ox}$ agents in the gaseous phase include atmosph anin $\mathrm{O}_{2}^{\prime} \overline{\mathrm{m}}$ xed with reducing alkyl boranes $[20,21]$, or less used, $\mathrm{H} 2$ (2) aturated ni-

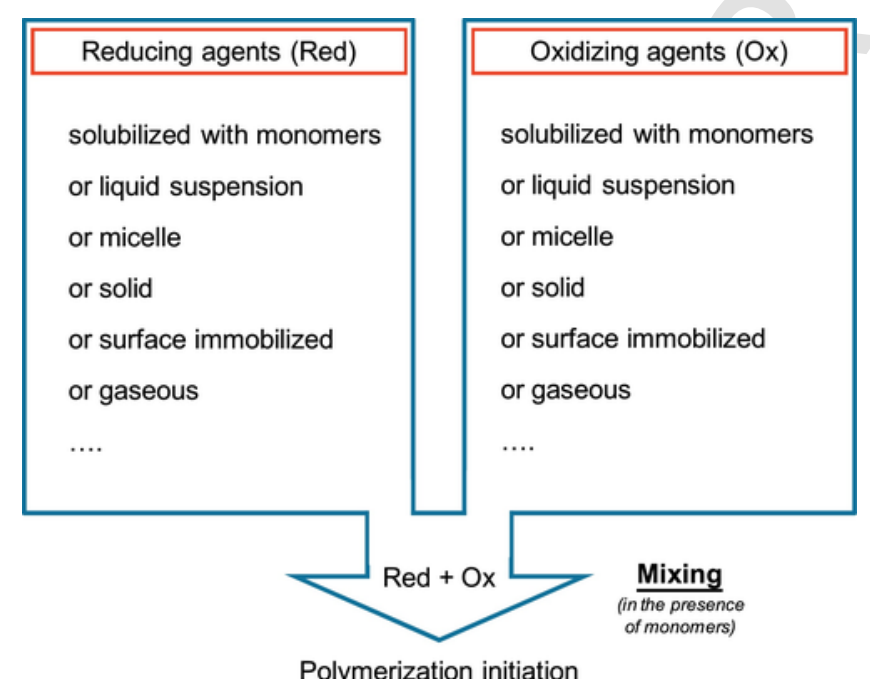

Scheme 2. Examples of phase speciation possible for oxidizing and reducing agents which will lead - through mixing - to the redox generation of polymerization initiating species (radicals or cations). trogen flux mixed in a solution containing monomers and oxalate esters [22]; it is also possible to find examples where Red is in the gas phase, tor example vapor state silanes in Crivello's redox CP (RCP)work $[$.

These varieties of combinations represent a formidable energetically efficient opportunity compared to other polymerization initiation; res esses. For example, if issues of stability are encountered for Ox wnen solubilized in a monomeric resin (second component), one can propose other configurations such as $\mathrm{Ox}$ in a liquid suspension (e.g., a few peroxides such as dibenzoylperoxide are stable at RT in water suspensions) or Ox directly stored in the solid form. From an engineering point of view, mastering the Red + Ox mixing conditions implies taking control of the polymerization initiation features, which can lead to widespread applications, such as polymer particles produced in suspension, surface grafting, composites production in the deepest part of a tooth in a dental cement, chemical anchoring systems, etc.

The mixing zone is important: providing good mixing of Red + Ox will lead to better redox polymerization efficiencies. For example, in Fig. 1, examples of a redox polymerization were monitored through optical pyrometry. In these examples, polymerization is carried out at room temperature and exothermicity is roughly proportional to the monomer conversion (here methacrylate $\mathrm{C}=\mathrm{C}$ conversion). A simple Pasteur pipette mixing of the methacrylate blenc lec to quite poor RFRP performances thanks to a benzoylperoxide( $\left.\mathrm{b}_{\mathbf{1}} \mathrm{v}\right) / \mathrm{Fe}(\mathrm{II}) / \mathrm{phos}-$ phine RIS with $50^{\circ} \mathrm{C}$ exothermicity reached (Fig. 1A, curve 1). Also, the produced polymer in bulk retained inhomogeneous features from the Pasteur pipette manual mixing. On the cont in Fig. 1A curve 2 , the use of a Sulzer Mixpac $($ mixing systems produced a homogeneous polymer with a very high polymerization efficiency $\left(100^{\circ} \mathrm{C}\right.$ exothermicity attributed to high $\mathrm{C}=\mathrm{C}$ conversion $)$.

The height of the sample for RFRP initiation also matters, as shown in Fig. 1B. Using a thin (4 mm) sample (curve 1), the BPO/ ferrocene initiating system is not efficient and no conversion was recorded (flat exothermicity). A polymer with a thin 1 'yus 1 surface was encountered after a $2 \mathrm{~h}$ reaction. On the contrary, for $\mathrm{y}$ thick sample (curve 2, same resin and concentrations, but $15 \mathrm{~mm}$ thickness) leads to slow version at the beginning $\left(23^{\circ} \mathrm{C}-40^{\circ} \mathrm{C}\right.$ in $\left.600 \mathrm{~s}\right)$, that is then spectacularly auto-accelerated $\left(100^{\circ} \mathrm{C}\right.$ in a few seconds). Accelerating effects in that case can be attributed to the reduced oxygen inhibition in a $15 \mathrm{~mm}$ thick setup [24,25]. Exothermicity is also significantly increased when higher masses are involved that will lead to thermal autoaccelerating effects [26]. Concepts involved in autoaccelerating effects are well known in FRP and will not be exhaustively treated here [27].

\subsection{Mild conditions for the initiation of a redox polymerization}

The main focus of this work is related to the redox initiating strategies carried out under mild conditions and without or with a low energy consumption (reaction at RT). Generally speaking, the initiation step of a polymerization reaction i.e. the first production of reactive species can be triggered by several strategies that are more or less energy efficient [29]: redox reactions, photopolymerization, Electron Beam (EB), ionizing radiations (e.g. gamma or X-rays), electrochemical reactions, thermal initiators, sonication [30,31]. Qualitatively, the energy consumption (ratio between energy provided against energy useful for the polymerization initiation) required for some FRP initiating strategies is presented in Scheme 3. Interestingly, redox initiation requires a very low energy consumption as it is a straightforward mixing of two components. Photopolymerization is 

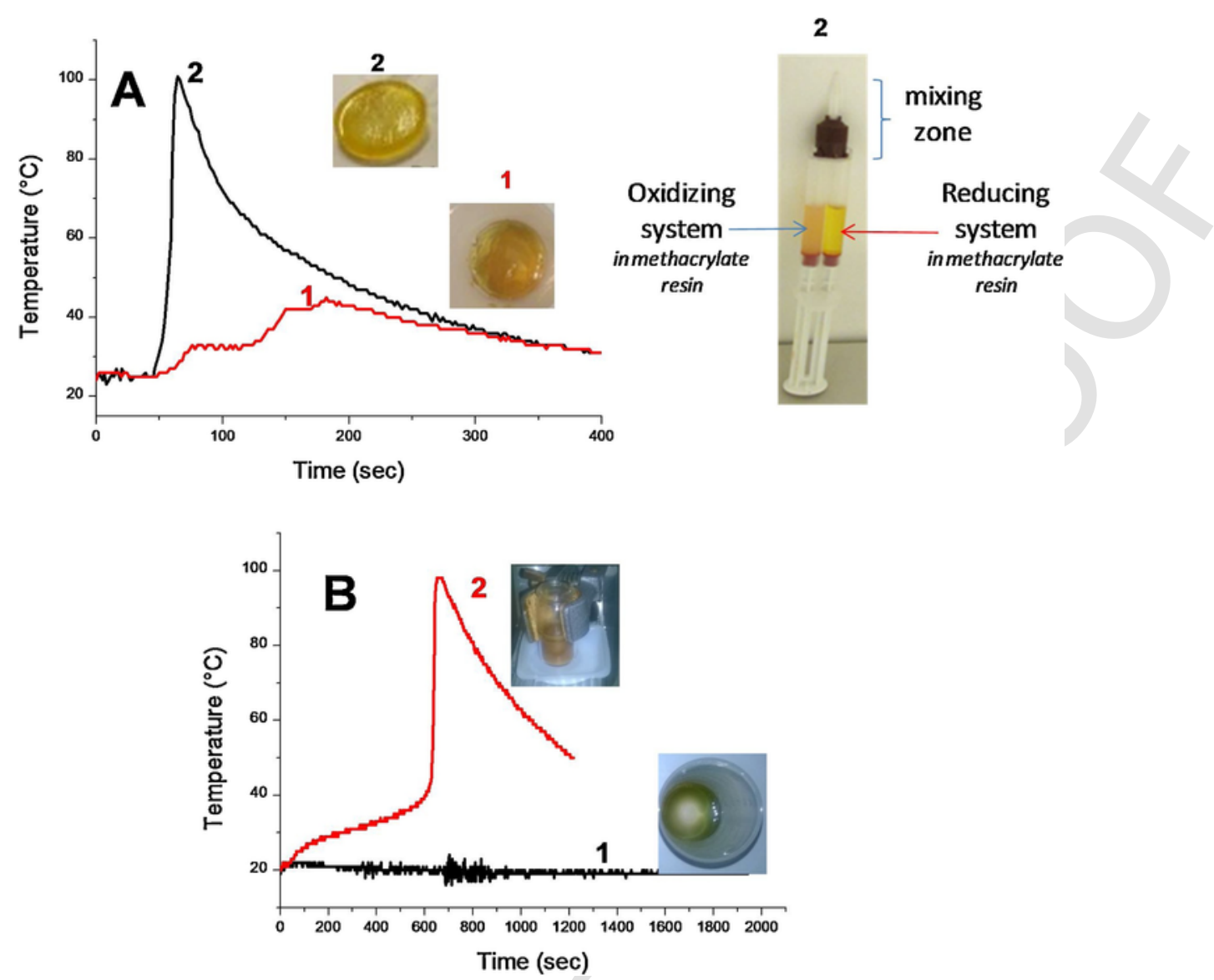

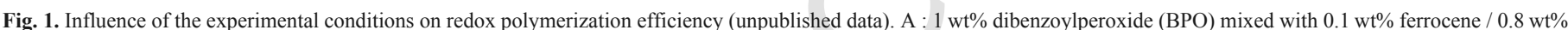

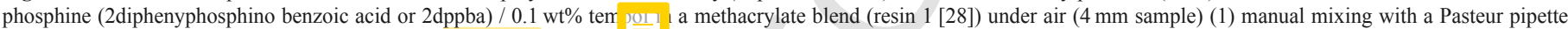

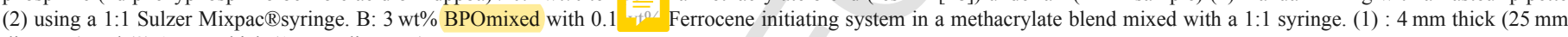
diameter) and (2) $15 \mathrm{~mm}$ thick (15 mm diameter).

\begin{tabular}{|l|l|l|}
\multicolumn{1}{|l|}{ photopolymerization } & & Thermal initiation \\
\hline redoxinitiation & EB curing & autopolymerization \\
\hline Energy consumption
\end{tabular}

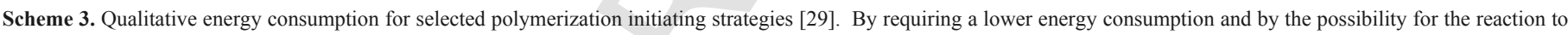

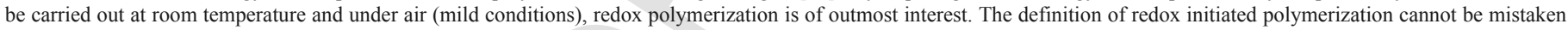
with polymer showing redox properties (useful in batteries, redox flow batteries...) [37,38].

also most of the time described as a low energy consumption strategy but this latter will obviously depend on the irradiation device (wavelengths and irradiances) but also of the involved irradiation times needed to achieve proper polymerization [32]. This might be an issue, especially for photocontrolled radical polymerization where polymer's synthesis times can exceed days [33]. Next, EB curing [34] - which is many times associated with UV-curing - requires average levels of energy inputs. Thermal initiation needs a complete (and energy inefficient) heating of the reaction medium in order to activate the thermal initiators. Finally, autopolymerization can occur for many monomers but, most of the time, this process starts at elevated temperatures. Some exceptions exist as exemplified with the pseudo Diels-Alder radical initiation that can occur when the reaction temperature reaches $90-120^{\circ} \mathrm{C}$ in the case of styrene [35,36]. Curing is sometimes used in applied polymer chemistry to describe hardening (toughening) of a monomer resin.
As in photopolymerization (e.g. laminated/air atmospheres present in the literature), there is no standard conditions defined as "mild conditions" in redox polymerization literature. Indeed, some initiating systems in the literature referred as redox initiating systems (see below) work in mildly heated medium $\left(25-45^{\circ} \mathrm{C}\right)$, with distilled monomers or under inert atmosphere. A strict and highly selective definition of mild conditions for redox polymerization is possible: room temperature, under air, no purification of the monomers, no additional energetic stimulus. This definition implies facing important challenges for the redox FRP initiation: i) the oxygen inhibition (under air) that consists in trapping carbon centered radicals formed at a very high quenching rate (diffusion-controlled reactions; see other reviews defining oxygen inhibition) $[24,39,40]$, ii) the room temperature $\left(20-25^{\circ} \mathrm{C}\right)$ conditions lowers significantly the dissociation constants of many reactants [8], iii) the propagation rate constants of a polymerization process is also lowered, iv) no energetic stimulus (such as electron beam or light $[32,34]$ ) are used to activate the reac- 
tivity and overcome the energetic barriers v) The phenols stabilizers present in vinyl resins can efficiently trap radicals [41]. Practically, many of our latest group's experiments were carried out at the bottom of a $25 \mathrm{~mm}$ diameter beaker having a thickness of about $4 \mathrm{~mm}$ (Scheme 4): it can be considered as a strict definition of mild conditions. Proposing efficient systems for strict mild conditions is a huge challenge in the polymer science [25].

In the present manuscript, more generally, we will focus on mild experimental conditions which are as close as possible to the room (or mild) temperatures $\left(0-45^{\circ} \mathrm{C}\right)$ where the redox initiation is of crucial interest as this approach requires almost no energy inputs. As in the other polymerization initiating techniques, curing $v s$. time profiles in redox initiated polymerization can be acquired by many monitoring strategies such as differential scanning calorimeter (DSC) [42], refractive index measurements [43], quantitative nuclear magnetic resonance [44-47], viscosimetry [48], real time Fourier transform infrared spectroscopy (RT-FTIR) [49], Raman (confocal) spectroscopy [50-52], optical pyrometry [53], thermal imaging [54,55].

\subsection{The art of gel time in redox polymerization}

Temporal control in redox polymerization processes is a function of the balance between the initiating and the inhibiting chemical reactions. In detail, in bulk RFRP for example, a two-component mixture is necessary in order to initiate the production of radicals. Immediately after mixing the two partners, propagation of FRP occurs (see below reactions $\mathrm{r} 1$ and $\mathrm{r} 2$ for an amine/peroxide initiation process, Scheme 5). The workability of the reaction is therefore inherently very low and this is a major issue for numerous applications: the mixture has to reach the deepest part (sometimes taking minutes) of the designated curing zone (e.g. in dental restorative materials). Therefore, radical trapping inhibitors were rapidly proposed in order to delay in time redox reactions (see $\mathrm{r} 3$ in Scheme 5). Gelation (Gel) time
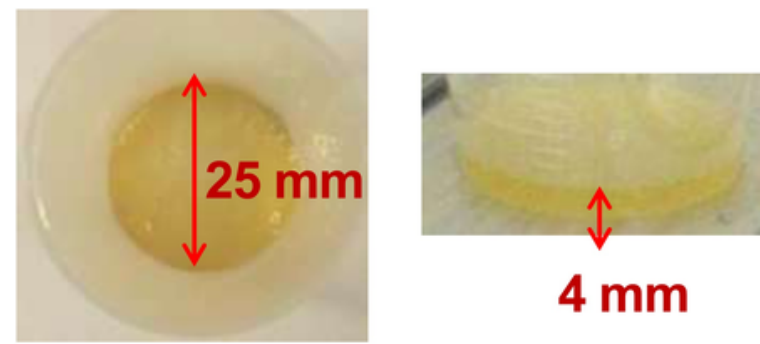

Scheme 4. Example of experimental setup that could be referred as strict mild conditions for redox polymerization initiation $\left(\mathrm{RT}, 23 \pm 2{ }^{\circ} \mathrm{C}\right.$, under air, no monomer distillation).

\section{Inititation (two components mixing) \\ amine + peroxide $\rightarrow \mathrm{R} \cdot$}

\section{Propagation}

$\mathrm{R} \cdot+\mathrm{nM} \rightarrow \mathrm{R}(\mathrm{M})_{n} \cdot$

Inhibition using phenols/nitroxide radical trapping agents

\section{$\mathrm{R}(\mathrm{M}) \mathrm{n}^{\bullet}+$ trap $\rightarrow$ Inactive species $\quad$ (delayed curing)}

Scheme 5. Classical initiation/propagation/inhibition reactions for the redox polymerization initiated by an amine/peroxide initiating system and delayed in time (longer gel time). Reproduced from ACS Omega. [56], Copyright 2018. Adapted with permission from the American Chemical Society. is then defined as the interval between mixing of the two-components redox system and the gel formation (then the material is no longer workable).

The radical trapping agents of reaction $\mathrm{r} 3$ (see Scheme 5) are commonly chosen among two families: phenols or nitroxide radicals. Indeed, the use of phenolic compounds as free-radical polymerization inhibitors is known since decades and these compounds are already present in the packaging of vinyl monomers (e.g. ppm levels of 4-methoxyphenol or MEHQ [41]). Phenols can also be used in order to delay in time a redox polymerization [57]. Nitroxides are the other class of compounds used in order to delay in time FRP $[58,59]$. Especially, many years ago, 4-hydroxy-2,2,6,6-tetramethylpiperidine 1 -oxyl (tempol) was demonstrated to have very interesting radical trapping properties - thus time delaying -for redox polymerization [60].

For example, in Fig. 2, tempol was used as an inhibiting/retarding strategy. One can see that without tempol (curve 1), the gel time is too fast (about $30 \mathrm{~s}$ ) and the workability of the system is very poor even if a strong conversion (namely, a $135^{\circ} \mathrm{C}$ exothermicity which is roughly proportional to the conversion [53]) is obtained. Therefore, classically, it is interesting to add $0.17 \mathrm{wt} \%$ tempol (curve 2): gel time was shifted to $200 \mathrm{~s}$ with still a $100^{\circ} \mathrm{C}$ exothermicity. The redox reaction was delayed in time thanks to the introduction of the nitroxide radical trapping agent. Upon addition of too much inhibitor/retardant $(0.34 \mathrm{wt} \%$ tempol, curve 3 ), the redox polymerization is completely inhibited. The choice of the suitable nitroxide concentration is therefore a key factor for good workability properties (3-5 min gel time or more) with still significant conversions. Also, fast conversions $v s$ time profiles are highly desired to get final conversions as high as possible.Therefore, considerable efforts to design efficient two or three components redox initiating systems have to be carried out in order to optimize all the reactants quantities. Typically, several design of experiments (DOE, trial and errors) are required, thus making the optimization of gel time an "art".

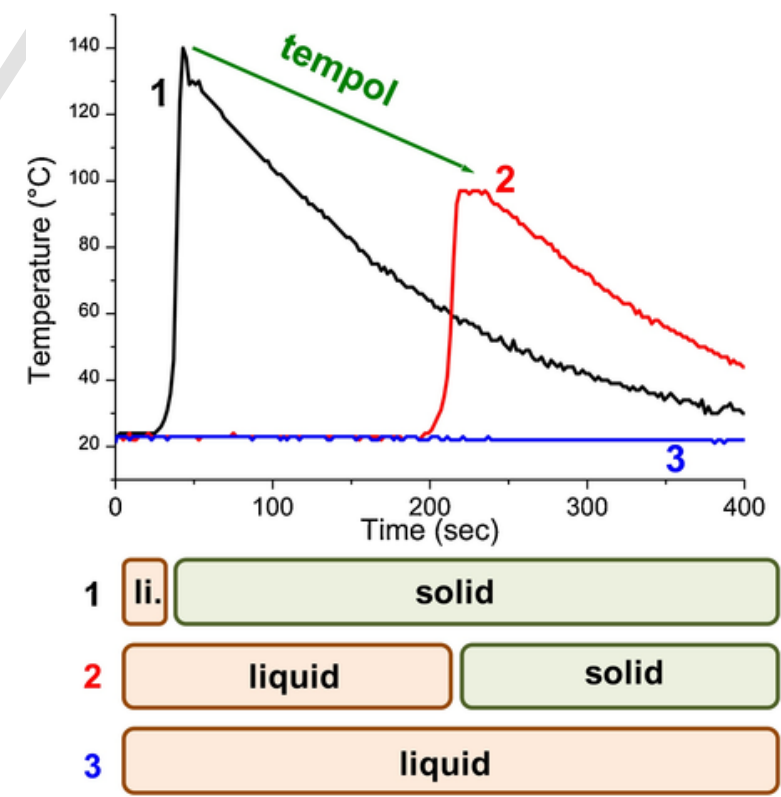

Fig. 2. Gel time optimization. Optical pyrometric measurements (Temperature vs. time) under air in a methacrylate blend (resin 1 , thickness $=4.0 \mathrm{~mm}$ ) for BPO $=1.0 \mathrm{wt} \%$ mixed with $0.9 \mathrm{wt} \% 4, N, \mathrm{~N}-\mathrm{TMA}$ and (1, too fast gel time) no tempol (li.: liquid); (2, optimum gel time) $0.17 \mathrm{wt} \%$ tempol and (3, complete inhibition) $0.34 \mathrm{wt} \%$ tempol [56]. Copyright 2018. Adapted with permission from the American Chemical Society. 
Marginally, other inhibiting techniques have been proposed in RFRP such as the slow release of the reducing agent through charge transfer complexes [56] and sulfonic acid esters as radical chain transfer agents [61]. Similar gel time optimizations are present in redox CP (RCP). In that case, gel time is also controlled by the balance between the production of superacids and the different cationic inhibitions (acid counter anion quenching, nucleophilic species quenching and presence of water).

\section{Initiating systems for redox FRP}

Free radical polymerization, as many chain-growth polymerizations, can be separated into three steps (Scheme 6 first, the radical production (initiation); second, the multiple reactions with monomers (propagation) and finally, the termination step and/or the quenching of the reactive radicals into inactive species. Presence of multifunctional monomers leads to cross-linking i.e. covalent bonding between macromolecules under growth. The next paragraph will include a few examples of the most popular redox FRP (RFRP) initiating strategies and a review of the technological limitations associated to these different approaches will be shortly and selectively discussed. The most popular and the most recent redox initiating reactions (with academic applications) by family (especially considering the oxidizing agent) will be presented.

\subsection{Peroxide-based initiating systems}

One of the easiest redox ways to generate free radicals (without light) is to reduce peroxides by metals. For example, Fenton's reagent, $\left(\mathrm{Fe}^{2+} / \mathrm{H}_{2} \mathrm{O}_{2}\right)[62,63]$ is quite efficient to generate hydroxyl radical ( $\mathrm{r} 1$, Scheme 7$)$. Precisely, an electron is transferred from iron (II) to the peroxide leading to a dissociation of the peroxide and the formation of iron (III). One clear obstacle to the widespre se of such a reaction is that the reducing metals can also reduc licals (r2) which lead to an inhibition of the reaction. Also, active alkoxy radicals can react with hydroperoxides in order to form inactive peroxyl radicals (r3). Therefore, too high concentrations of metals and peroxides increase the probability for the inhibiting mechanisms to occur and fine tuning of the radical generation appears to be more difficult [64]. Practically, initiating FRP of organic monomers using iron(II)/peroxides systems is difficult under mild conditions (see defi-

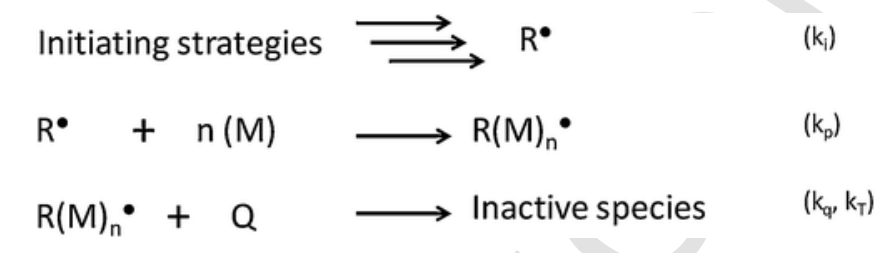

Scheme 6. Simplified initiation, propagation and termination/quenching mechanisms occurring in free radical polymerizations (FRP). nition vide supra) [65-68] and the studies currently presented in the literature require the reaction medium to be heated (or the use of inert atmospheres and/or very long reaction times e.g. more than four hours for Kalenda et al. using a ferrocene/BPO RIS [69]).

Other metallic reducing agents reported in the literature include (but are not limited to) $\mathrm{Ag}(\mathrm{I}), \mathrm{Cu}(\mathrm{I}), \mathrm{Rh}(\mathrm{I}), \mathrm{Mn}(\mathrm{II}), \mathrm{Co}(\mathrm{II}), \mathrm{V}(\mathrm{V})$ [8,70-75]. More recently, Garra et al. conducted a study using a copper (I) complex inducing the decomposition of dibenzoyl peroxide (BPO) in the presence of ascorbic acid (VitC) as the reducing agent (catalytic cycle) [76]. Different structure/reactivity/efficiency relationships in RFRP were proposed according to the ligands present on the $\mathrm{Cu}(\mathrm{I})$ center. They are represented in Scheme 8: i) oxidation potential of $\mathrm{Cu}(\mathrm{I})$ complexes will have an important influence on RFRP gel times when ii) it is the $\mathrm{Cu}(\mathrm{II})+\mathrm{VitC}$ reaction i.e. the reversibility of $\mathrm{Cu}(\mathrm{I}) \rightarrow \mathrm{Cu}$ (II) oxidation process that will have the most important impact on the RFRP efficiency. Similarly, these two criteria were found in the literature for efficient photocatalysts (e.g. copper (I)) in photopolymerization initiation: oxidation potential of the $\mathrm{Cu}(\mathrm{I})$ was - through $\Delta \mathrm{G}$ calculations $[77,78]$ - present in the photocatalyst efficiency criteria. Also, the reversibility of the oxidation process is a key parameter for (metal or metal-free) photocatalysts [77,79]. Hydroperoxides $(\mathrm{ROOH})$ can also be generated in situ from the oxygen inhibition during a photopolymerization carried out under low light intensity. These latter can next be decomposed through redox mechanisms (by a $\mathrm{Cu}(\mathrm{I}) /$ reducing agent system) forming an original one component redox system robust in shadowed areas (also referred as lateral photopolymerization) [79].

In the case of organic resins, one of the most efficient reference for redox polymerization is based upon th ction of $\mathrm{N}$-aromatic amines with dibenzoyl peroxide (BPO) disco 1 in the 1950 s ! [80,81] It is still applied in many polymerization processes [7,8,19,82-84] and can find use in many high-tech applications requiring in-depth polymerization [85-89]. The oldest mechanism proposed that the amine radical cation is the major responsible for initiation [80]. But recent works confirmed the generation of $\mathrm{Ph}(\mathrm{C}=\mathrm{O}) \mathrm{O}^{*}, \mathrm{Ph}^{*}$ and aminoalkyl radical species (Scheme 9) $[83,90]$. Substitution effect on the amine reactivity was already studied in the early work by Horner and Scherf [80] and 4- $N, N$ trimethylaniline (4- $N, N$ TMA) was found to be an excellent reactive species for this reaction. It is still a hot topic today [91].

Still using peroxides as oxidizing agents, bulk redox polymerization of trimethylolpropanetriacrylate (TMPTA, here distilled) was proposed by Ermosl inim et al. thanks to a remote hydrogen peroxide $\left(\mathrm{H}_{2} \mathrm{O}_{2}\right)$ / tetralky] aryl) borate $\left(\mathrm{R}_{4} \mathrm{~B}^{-}\right)$initiating system [92]. In that experiment, nitrogen gas was saturated by $\mathrm{H}_{2} \mathrm{O}_{2}$ vapors and then flowing towards a flask containis g tl e borate anion in the bulk monomer. Solubility issues of for the vurate salt required $10 \%$ of solvent to improve the dispersion of the borate salt in the reactive medium. As illustrated in Scheme 10, the reaction involved the reduction of $\mathrm{H}_{2} \mathrm{O}_{2}$ thanks to the oxidation of the borate anion; it leads to the formation of hydrogen peroxide radical anion that undergoes a rapid dissociation (as BPO above) to form a hydroxyl anion and an-

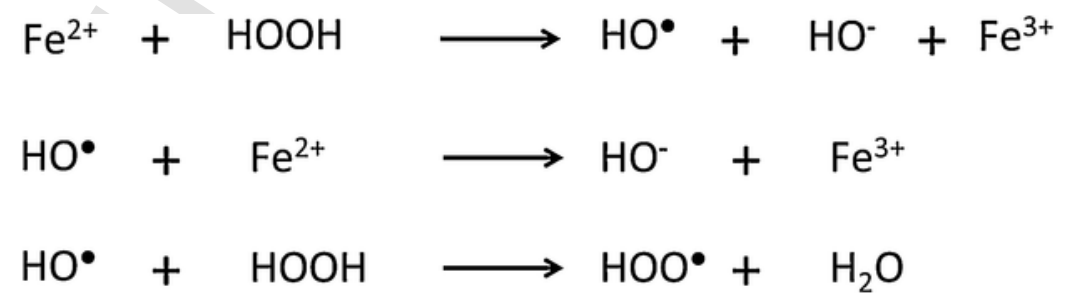




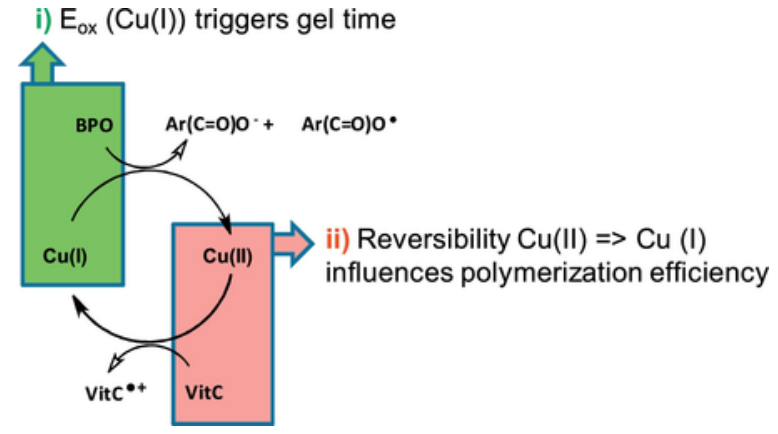

Scheme 8. Proposed efficiency criteria for the $\mathrm{Cu}(\mathrm{I})$ in $\mathrm{Cu}(\mathrm{I}) / \mathrm{VitC} / \mathrm{BPO}$ RFRP initiating systems.

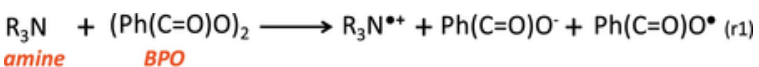

$$
\begin{aligned}
& \mathrm{R}_{3} \mathrm{~N}^{\bullet+}+\mathrm{Ph}(\mathrm{C}=\mathrm{O}) \mathrm{O}^{-} \longrightarrow \mathrm{R}^{\prime} \mathrm{C}^{\bullet} \mathrm{NR}_{2}(-\mathrm{H})+\mathrm{Ph}(\mathrm{C}=\mathrm{O}) \mathrm{OH} \quad(\mathrm{r} 2) \\
& \mathrm{Ph}(\mathrm{C}=\mathrm{O}) \mathrm{O}^{\bullet} \quad \longrightarrow \mathrm{Ph}^{\bullet}+\mathrm{CO}_{2}
\end{aligned}
$$

Scheme 9. Redox radical initiating system using amine / diber toys peroxide (BPO) interaction (Adapted from $[83,90]$ ). R' represents one of the an ine ubstituents without the alpha carbon of the nitrogen and its labile hydrogen and its labile hydrogen (see detailed mechanism in the publication by Zoller et al.[82]).

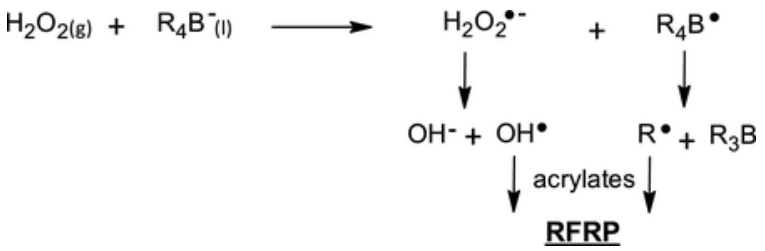

Scheme 10. Hydrogen peroxide/trialkyl (or aryl) borate $\left(\mathrm{R}_{4} \mathrm{~B}^{-}\right)$redox FRP (RFRP) initiating system as described by Ermoshkinet al. [92], Copyright 2008. Adapted with permission from the American Chemical Society.

other hydro $\bar{y}$ adical. Also, the borate anion oxidation leads to a tetralkylboron radical that can produce an alkyl radical and a trialkylborane. Interestingly, under air (not present in the study), the trialkyl boranes generated are likely to react with oxygen in an aerobic atmosphere (see below). Similar reactions and setups were proposed by the same group using oxalate esters in combination with $\mathrm{H}_{2} \mathrm{O}_{2}(\mathrm{~g})\left(\mathrm{N}_{2}\right.$ vapor phase) [22]. Again, in both examples, inert (nitrogen or argon) atmosphere and distilled monomers were used.

Finally, reactivity of peroxides is strongly related to their reduction potentials (and BDEs). For example, BPO ( $\mathrm{E}_{\text {red }}=+0.27 \mathrm{~V} v s \mathrm{SCE}$ [93]) is most of the time more reactive $[80,86,87,94]$ than tert-butylperbenzoate $\left(\mathrm{E}_{\mathrm{red}}=+0.18 \mathrm{~V}\right.$ vs SCE [95]) which is more reactive than cumenehydroperoxide $\left(\mathrm{E}_{\text {red }}=+0.08 \mathrm{~V} v s\right.$ SCE [93]). Experimental conditions such as the $\mathrm{pH}$ can also have a strong influence on the dissociation behavior of peroxides [55,96-98]. Noteworthy, too low BDEs in peroxides lead to low self-accelerating decomposi-

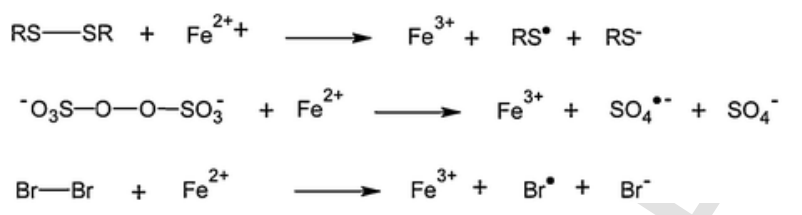

Scheme 11. Example of redox initiating reactions where weak bonds are reduced by $\mathrm{Fe}(\mathrm{II})$. Adapted from Sarac et al. [8], Copyright 1999. Adapted with permission from Elsevier Ltd.

tion temperatures (SADT) which is a good scale to represent the peroxides stability [99].

\subsection{Other weak bonds: disulfides, persulfates ...}

Next, compounds bearing a weak BDE bond also include (but are not limited to) persulfates, dihalogens (e.g. $\left.\mathrm{Br}_{2}\right)$, disulfides, peroxomonosulfates, peroxodiphosphates, [8] ... Many weak bonds can be reduced as exemplified in the Scheme 11: persulfates, disulfides or dihalogens can readily produce radicals when these latter are mixed with efficient reducing agents [100-104]:

Interestingly, the presence of two phases during the polymerization in emulsion allowed to solubilize ammonium persulfate in the water phase and by smart combination of i) reducing agents and ii) experimental conditions (surfactant concentration) efficient RFRP in emulsion can be initiated [105-111]. Compared to BPO, potassium persulfate is particularly interesting for biomedical applications [112]. Persulfate/bisulfite and persulfate/ Bruggolite FF6 initiating systems are widely used in industry [113].

\subsection{Metal/reducing agents: $\mathrm{Ce}(\mathrm{IV})$}

Instead of weak bonds oxidizing agents, Ce(IV) (ammonium, sulfates and/or nitrates) can be reduced to $\mathrm{Ce}$ (III) with many reducing agents such as alcohols [114,115], aldehydes, acids, amines [116], and more generally chelating agents in order to form free radicals [7,8,117-119]. Most of the mechanisms reported in the literature propose first the formation of an intermediate Ce(IV) complex from $\mathrm{Ce}(\mathrm{IV})$ that can be subsequently dissociated into three species, leading to a radical formation on the chelated adduct and the removal of a labile hydrogen and the formation of Ce(III). For example, in Scheme 12 , we illustrated such a reaction while using 2-chloroethanol as a reducing agent [120-122].

Rates of the radical generation for the Ce(IV)/reducing agents systems remain limited for many of the systems reported in the literature and many examples required specific conditions for the polymerization of monomers (e.g. distilled acrylonitrile/acrylamides) and/or slight heating (and sometimes inert atmospheres) [123-125]. Another issue is that the propagating radicals can react with $\mathrm{Ce}(\mathrm{IV})$ leading to a termination reaction. More recently, $\mathrm{Ce}$ (IV) was also used to functionalize a graphene oxide surface using methylmethacrylate, acrylonitrile and acrylamide in the presence of acids (Scheme 13) [126]. Such a reaction shows the versatility of the redox reactions and the

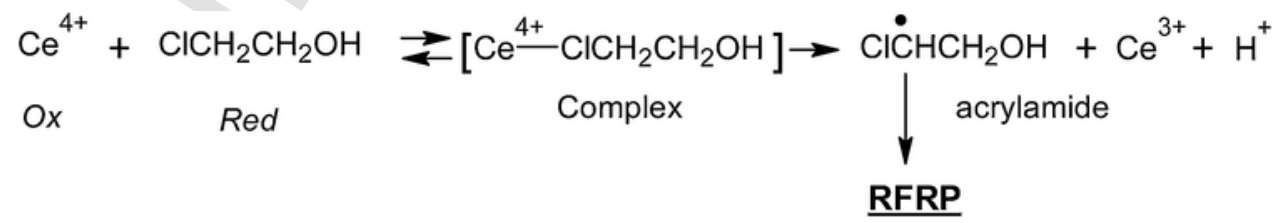

Scheme 12. RFRP initiation using a Ce(IV)/reducing agent initiating system as described by Renders et al. [115]. 

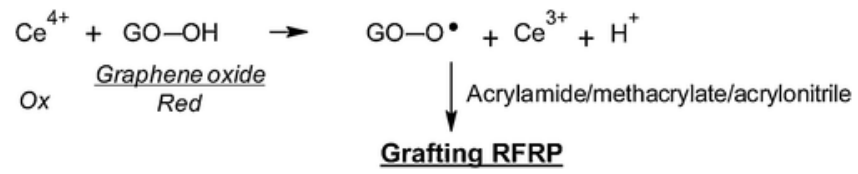

Scheme 13. Graphene oxide $(\mathrm{GO}-\mathrm{OH}) / \mathrm{Ce}(\mathrm{IV}) / \mathrm{HNO}_{3}$ redox initiating system (at $\left.30^{\circ} \mathrm{C}\right)$ as described by Ma et al. [126], Copyright 2013. Adapted with permission from Elsevier Ltd.

possibility to functionalize highly interesting graphene surfaces [127-130].

\subsection{Metal/reducing agents part $B: M n, C u$ and other metals}

Close enough to the $\mathrm{Ce}(\mathrm{IV}) /$ reducing agent reactions previously mentioned, the $\mathrm{Mn}(\mathrm{III}) /$ reducing agent initiating systems should also be mentioned [8]. An exhaustive review concerning these systems is available in the literature by Snider [131] with the use of Manganese $($ and $\mathrm{Cu})$ in organic free radicals chemistry. In organic synthesis, reactions are generally carried out under demanding conditions (inert atmosphere or thermal heating) and the radical generating rates are generally not comparable with what is necessary for FRP. Therefore, this paragraph will only focus on what is available in the very early literature for radical polymerization initiation. For instance, Zheng et al. reported the RAFT polymerization of methyl methacrylate (MMA) using simply $\mathrm{Mn}(\mathrm{acac})_{3}$ to generate radicals at $25^{\circ} \mathrm{C}$ [132]. However, the reaction conditions were harsh: several hours were necessary to get a significant monomer conversion, an inert atmosphere was also required and use of purified MMA in order to remove any stabilizer inhibition was also necessary. Other reports indicate that harsh solicitations [133] were required with $\mathrm{Mn}(\mathrm{acac})_{3}$ to generate acac* radicals: UV, gamma-ray or even high temperature $\left(100{ }^{\circ} \mathrm{C}\right)$. In these conditions, the reaction between $\mathrm{Mn}(\mathrm{acac})_{3}$ and organic halides was still slow ( $1 \mathrm{~h}$ to reach $72 \%$ of conversion for the halogenated alkyls at $100{ }^{\circ} \mathrm{C}$ ). Parallel to this, the polymerization of methyl methacrylate with $\mathrm{Mn}(\mathrm{acac})_{3}$ in the presence of organic halides was also reported but at lower temperature i.e. $80^{\circ} \mathrm{C}[134,135]$. Some study also reported the use of $\mathrm{Mn}(\mathrm{acac})_{3}$ in combination with triphenylphosphine (tpp) for the polymerization of styrene but this study was carried out at $100^{\circ} \mathrm{C}$ [136]. Carboxylic acids were also considered as potential reducing agents in the literature, for example, in the $\mathrm{Mn}(\mathrm{acac})_{3} /$ acetic acid initiating system [137] which remains an extremely slow system (200 min for the polymerization of alkyd resins) or under argon $[107,138]$. By examining a wide range of acids for RFRP, a series of Mn(III)/carboxylic acids combination were examined and the following reacti lity scale could be established, for reactions carried out under inte. ....nosphere and at room temperature [139]: citric $>$ tartaric $>$ ascorbic $>$ oxalic $>$ succinic $>$ glutaric $>$ adipic acid. The robustness of such radical initiating systems under "strict mild conditions" was not tested in this study.

These different mitigate initiated systems motivated the development of new combinations such as amine-free peroxide-free RFRP initiating systems based on $\mathrm{Mn}$ or $\mathrm{Cu}$ in combination with phosphines as reducing agents. Strictly mild conditions (Scheme 4) were used for the RFRP initiation of a low viscosity methacrylate blend. In the Table 1 (entry 1 and 2), a good estimation of the impact of the mild conditions on the RFRP initiation can be performed: the early works were carried out under harsh conditions - see above (entry 1) - and citric acid can be considered as a good reducing agent (inert atmosphere) as in Table 1 (entry 2), but this reducing agent is inefficient under strictly mild conditions. Adversely, Metal Acetylacetonates - Bidentate Ligand Interaction (MABLI) radical generating reactions (entries 4, 6 and 7) showed $\mathrm{Mn}(\mathrm{III})$ (or $\mathrm{Cu}(\mathrm{II})$ or $\mathrm{V}(\mathrm{III})$ )/ 2-diphenylphosphino benzoic acid (2dppba) initiating systems are highly efficient for RFRP initiation under strictly mild conditions. These systems were notably competitive with the 4-N,NTMA/BPO initiating system especially for an efficient polymerization at the surface of the sample due to the presence of phosphine [140]. MABLI was then considered using several metal acetylacetonates varying by the nature of the ligand substitution [17]. Parallel to this, the variation of the aryl substituent on $2 \mathrm{ddpba}$ was also examined $[49,141]$. Other metals are also proposed as oxidizing agents, such as $\mathrm{Cr}(\mathrm{VI}), \mathrm{Mn}(\mathrm{IV})$, Ti(III) [143] or $\mathrm{Cu}(\mathrm{II})[8,144]$ (Scheme 14).

\subsection{Other initiating systems (alkylborons, iodoniums,...)}

Next, in the presence of oxygen, alkylborons can react with $\mathrm{O}_{2}$ in order to initiate a redox polymerization at room temperature (Scheme

Table 1

Illustration of Metal complexes/reducing agents RFRP initiating efficiency under different conditions.

\begin{tabular}{|c|c|c|c|c|c|c|}
\hline Entry & Mt complex & Reducing agent & Atmospheric conditions & Monomers & Polymerization Efficiency (RT) & Ref \\
\hline 1 & Mn (III) sulfate & Citric acid & $\mathrm{N}_{2}$ degassed & Acrylonitrile (purified) & +++ & {$[139]$} \\
\hline 2 & $\operatorname{Mn}(\mathrm{acac})_{3}(\mathrm{III})$ & Citric acid & Air (4 mm) & methacrylate blend & n.p. & {$[141]$} \\
\hline 3 & $\mathrm{Mn}(\mathrm{OAc})_{3}(\mathrm{III})$ & $2 \mathrm{dppba}$ & Air (4 mm) & methacrylate blend & n.p. & {$[141]$} \\
\hline 4 & $\operatorname{Mn}(\mathrm{acac})_{3}(\mathrm{III})$ & 2dppba & Air (4 mm) & methacrylate blend & +++ & {$[141]$} \\
\hline 5 & $\mathrm{Cu}(\mathrm{OAc})_{2}(\mathrm{II})$ & $2 \mathrm{dppba}$ & Air $(4 \mathrm{~mm})$ & methacrylate blend & n.p. & {$[49,141]$} \\
\hline 6 & $\mathrm{Cu}(\mathrm{acac})_{2}$ (II) & $2 \mathrm{dppba}$ & Air (4 mm) & methacrylate blend & +++ & {$[17,49,141]$} \\
\hline 7 & $\mathrm{~V}(\mathrm{acac})_{3}(\mathrm{III})$ & 2 dppba & $\operatorname{Air}(4 \mathrm{~mm})$ & methacrylate blend & + & {$[141,142]$} \\
\hline
\end{tabular}

Other metals are also proposed as oxidizing agents such as $\mathrm{Cr}(\mathrm{VI}), \mathrm{Mn}(\mathrm{IV}), \mathrm{Ti}(\mathrm{III})$ [143] or $\mathrm{Cu}(\mathrm{II})[8,144]$.

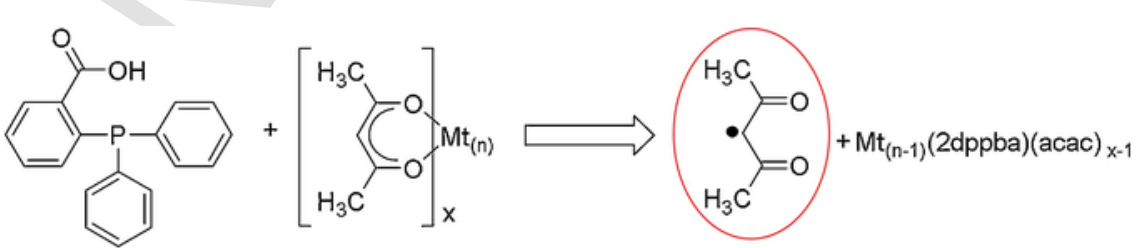

Bidentate ligand

Metal acetylacetonate

Free radical generation

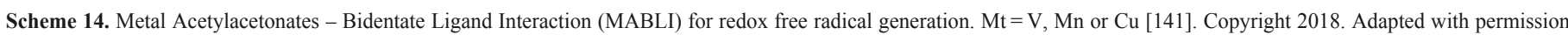
from the Royal Society of Chemistry. 
15) [20]. However, a strict control of the oxygen concentration and the alkyl boron species is required to obtain reproducible results [21]. The metal/ $\mathrm{R}-\mathrm{Cl}$ reaction (e.g. $\mathrm{Ni}$, Mo, Pt with $\mathrm{CCl}_{4}$ ) was also considered for initiation but the different reports mention the use of inert atmospheres and the distillation of the monomers prior reaction; it is thus difficult to compare the radical generating rates with the mild conditions described above [145]. Another patent [146] also states the use of an aldehyde/Co(II) system where hydroperoxides are generated in situ under air but, to the best of our knowledge, the corresponding chemical mechanisms were not disclosed.

Contrary to the photopolymerization initiators, only scarce examples of iodonium salts can be found as RFRP initiating systems. However, iodonium salts offer many advantages. For example, they are stable in resins and some derivatives are even biocompatible (e.g. they are used in dental restorative materials $[147,148])$. As RFRP initiator, Tehfe et al. mixed an iodonium salt (Iod) with a $N$-heteroarylborane showing a low oxidation potential $\left(\mathrm{E}_{\mathrm{ox}}=0.5 \mathrm{~V} v s \mathrm{SCE}\right)$. This resulted in the reduction of the iodonium salt generating Iod ${ }^{\bullet}$ as the radical that rapidly dissociates in a few hundreds of picoseconds [149] into highly reactive aryl radicals (Scheme 16). These latter were then harvested for the RFRP of trimethylolpropanetriacrylate (TMPTA). It is well known that methacrylates are less reactive than acrylates, but these monomers are also less toxic, which makes their RFRP initiation more difficult. Very recently (Scheme 16), it was still possible to induce the polymerization of a methacrylate blend (RT, under air) using an iodonium salt in combination with an amine having a very low oxidation potential (tris[4-(diethylamino)phenyl]amine (t4epa), $\mathrm{E}_{\text {ox }}=-0.05 \mathrm{~V}$ vs SCE [150]).

Finally, the excellent study by Broggi et al. showed that it is possible to consider a few examples of monomers (acrylics, acrylamides, acrylonitriles...) as the oxidizing agents [152]. Indeed, the organic electron donors (OED) are characterized by extremely low oxidation potentials $(-1.2 \mathrm{~V} v s \mathrm{SCE})$, rendering these OED capable to reduces the monomers; nevertheless they also demonstrated that as a double oxir ats $n$ of the OED was observed, the redox reaction leading mostly $\checkmark$ un anionic chain growth polymerization (radical formed on the monomer being reduced to an anion) rather than to a FRP reaction.

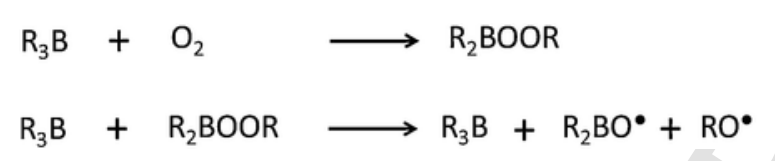

(r1)

Scheme 15. Redox radical initiating system using alkyl boron $\left(R_{3} B\right)$ /oxygen interaction [21]. Copyright 1962. Adapted with permission from John Wiley \& Sons Inc.

\subsection{Monomers and other features in redox FRP}

\subsubsection{Monomers used in RFRP}

Virtually, all the monomers polymerized by FRP processes can be polymerized by RFRP (Scheme 17). For example, styrene or acrylamide moieties can be used [153]. Similarly, acrylates, with their very high propagating rate constants [154], show extremely good compatibility with RFRP processes. As reported by Zoller et al.,[83] molecular weight $v s$. conversion profile are following the kinetics of the redox initiating reaction. Multifunctional monomers can lead to crosslinking i.e.the formation of a dense 3D network connected by covalent bonding which is particularly useful in adhesives, biomaterials, chemical anchoring... The choice of the monomers will depend on the final applications. Some monomers also require very high radical generating rates in order to be efficiently cured, e.g. the propagation constants of methacrylates are roughly ten times lower than that of the acrylate ones. A broad diversity of the redox initiating systems is required to scope with broad RFRP conditions; high radical generating rates, good stability and good solubility being the three most important features for the Red and Ox compounds.

\subsection{The oxygen inhibition in RFRP}

One of the most important challenge of redox FRP initiation under mild conditions and under air is the oxygen inhibition. Rapidly, in liquid/viscous resins commonly used in FRP, atmospheric oxygen $\left(\mathrm{O}_{2}\right)$ is easily dissolved. The classic study by Bowman et al. [39] measured a concentration of $1.2 \mathrm{mM} \mathrm{O}_{2}$ dissolved in 1,6-hexanediol diacrylate at ambient temperature and pressure. This ground state triplet oxygen reacts at a very high quenching rate (diffusion-controlled reaction) with many radicals involved in the FRP processes ( $\mathrm{r} 1$ and $\mathrm{r}^{\prime}$, Scheme 18) resulting in the production of poorly reactive peroxyl radicals (ROO* ). These latter $\mathrm{ROO}^{*}$ radicals are indeed poorly reactive as they have a marked $\mathrm{O}-\mathrm{O}$ delocalization using lone pair on both oxygen atoms. It is also illustrated by the bond dissociation energy of RO- $\mathrm{H}$ which is much higher than that the one of ROO-H [140]. Quenching of the active radicals leading to the formation of inactive radicals or species (such as (hydro)peroxides, r3) is commonly referred as "oxygen inhibition".

Once initially solubilized $\mathrm{O}_{2}$ reacts and inhibits FRP, Bowman et al. [39] showed that the main oxygen inhibition issue/mechanism in FRP is caused by the back diffusion of oxygen inside the reaction medium (from the atmosphere back to the formulation). Consequently, the polymerization of viscous resins is easier under air than for fluid ones. This will result in excellent in depth curing for RFRP
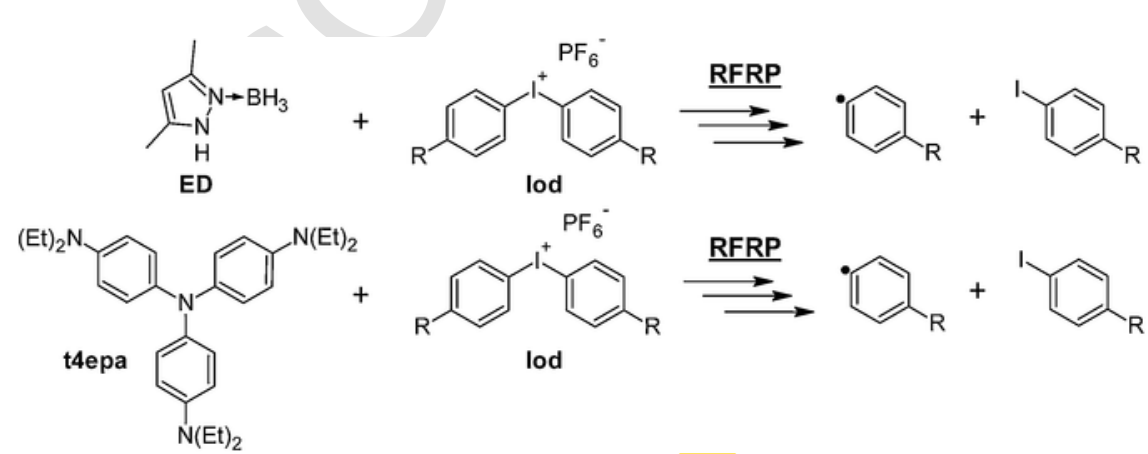

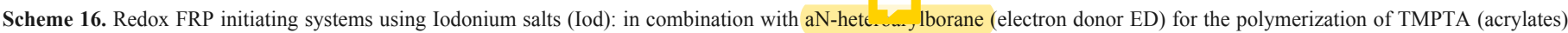

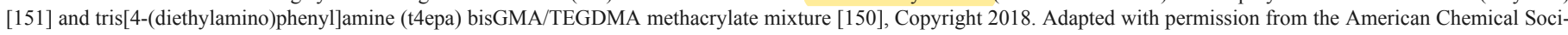
ety. 

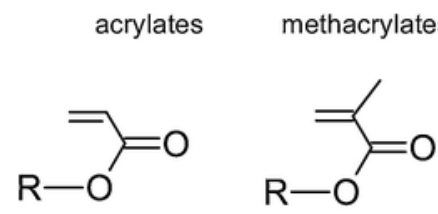

$\operatorname{Red}+\mathrm{Ox}$

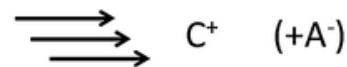

(redox)

Scheme 19. General initiating strategy for redox cationic polymerization (RCP) initiation thanks to initiating cations $\left(\mathrm{C}^{+}\right.$, with associated counter anions, $\left.\mathrm{A}^{-}\right)$. Red and $\mathrm{Ox}$ stand for reducing system and oxidizing system, respectively.

Scheme 17. Examples of monomers that can be functionalized using RFRP initiating systems.

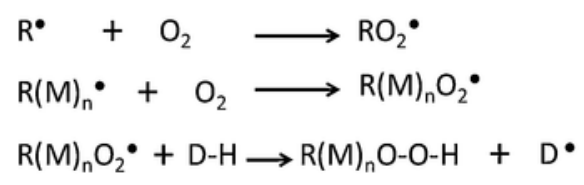

Scheme 18. Oxygen inhibition in FRP.

but tacky surfaces in many RFRP polymers synthesized under air. Tacky surface is a term used in applied polymer chemistry to describe the liquid layers (from oxygen inhibition) present at the surface of many polymers. Classical strategies to reduce the oxygen inhibition in FRP have been already reviewed in the literature $[24,25,40]$.

\subsection{Shrinkage in RFRP}

Another clearly identified drawback of RFRP processes is the shrinkage inherently present in the $\mathrm{C}=\mathrm{C}$ double bond conversion into $\mathrm{C}-\mathrm{C}$ single bond (e.g. due to Van der Waals intermolecular forces reduction [155]). The polymer has a reduced space volume compared to the monomers in FRP - this process is referred as "shrinkage". Evaluation of the shrinkage can be performed using thermo-mechanical analysis, rheometers, pycnometers [156] or more recently micro-tomography [157]; each measuring technique is adapted to different kinetic profiles. Due to the variety of sample sizes, shapes, polymerization stress, curing temperatures and intermolecular forces in the monomers, many shrinkage values can be found in the literature (e.g. $1-16$ vol $\%$ for acrylates) $[156,158]$.

\section{Initiating systems for redox $\mathrm{CP}$}

Cationic polymerization (CP) also represents a significant quantity of the chain-growth produced polymer [32], quite important in epoxy curing [159]. Generally speaking, polymer networks produced by CP show a better adhesion than those obtained by FRP though the polymerization is slower than for the free radical one and the choice of monomers remains currently more limited. Strategies for the CP initiation are indeed very close to those developed for the FRP ones; their energy requirements are therefore in the same range of magnitude than that required for FRP. The general goal is to obtain Brønsted or more generally Lewis acids from an initial stimulus. Their production (initiation) can be performed from a redox reaction occurring when two components are mixed (See Scheme 7) [23], light initiated [32], EB cured [34] or also cationic thermally initiated (e.g. pyrylium salts [160]). More generally, the decomposition of onium salts leads to a very efficient production of superacids $\left(\right.$ e.g. $\left.\mathrm{H}^{+}, \mathrm{PF}_{6}{ }^{-}\right)$. [23,161-165] We will focus in the present paragraph on the redox CP (RCP) initiations (Scheme 19).

Compared to FRP, the cationic polymerization shows - in general - slower polymerization kinetics (and sometimes higher induction period) for the propagation of cationic species [167]. Indeed, intrinsically, cationic species show much better diffusion constants than radicals as they have higher lifetimes (e.g. $\mathrm{H}^{+}, \mathrm{A}^{-}$can be stable). Shrink- age is much more limited in CP compared to FRP [167-169]. It has to be related to the reduction in space from the monomers to the polymer (e.g. Van der Waals forces intermolecular repulsing forces are higher for monomers than for polymers). An example of cationic propagation is presented in Scheme 20. In that example, the generated acids are trapped by oxygen lone pairs of oxirane leading to the ring opening of the epoxide. The resulting cations can subsequently react with other monomers in a propagation step. Here, we can clearly see that counter anions ( $\mathrm{A}^{-}$, if present in the acids) have a huge impact on the $\mathrm{CP}$ propagation as the reactivity (acidity) of the cationic center will mainly be driven by them. For example, triflate anions will be characterized by much lower propagation constant rates compared to those obtained with the highly delocalized tetrakis(pentafluorophenyl) borate anions $\left(\mathrm{B}\left(\mathrm{C}_{6} \mathrm{~F}_{5}\right)_{4^{-}}\right.$, in Rhodorsil PI2074 [23]). Crivello et coworkers showed that, acids reactivity in $\mathrm{CP}$ follows $\mathrm{A}^{-}$nucle-

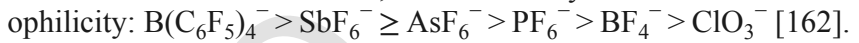

In the present paragraph, we will survey the RCP initiating reactions i.e. the ones responsible for the generation of cationic superacids. At first and particularly, $\mathrm{Cu}$ in combination with onium salts will be detailed in a first development (3.1). Secondly, other methods involving other dissociation ways for the onium salts into superacids will be explored (3.2). Finally, a few examples among the CP monomers currently proposed will be considered.

\section{1. $C u(I I) / C u(I) R C P$ initiating systems}

Deciphering the chemical reactions that initiate the RCP using a $\mathrm{Cu}$ (II)/reducing agent/iodonium salt system was not straightforward in the literature [170-172]. First, it is admitted that $\mathrm{Cu}$ (II) reacts with the reducing agent to form in situ $\mathrm{Cu}(\mathrm{I})$ (Scheme 21). Mechanistic study around the $\mathrm{Cu}(\mathrm{I})$ decomposition of iodonium salts (without energy and at RT) remains unsettled. Several studies showed that the production of radicals useful for the RFRP is very limited (r2), and that probably a complexation of the iodonium salt by the $\mathrm{Cu}(\mathrm{I})$ can lead to a $\mathrm{Cu}(\mathrm{III})$ intermediate having a short lifetime. Nevertheless all the studies showed that the iodonium salt counter anion is also present on the generated cations (e.g. $\left.\mathrm{H}^{+}, \mathrm{PF}_{6}{ }^{-}\right)$. These latter can be described as superacids. Also, the oxidation potential of the $\mathrm{Cu}(\mathrm{I})$ gen-

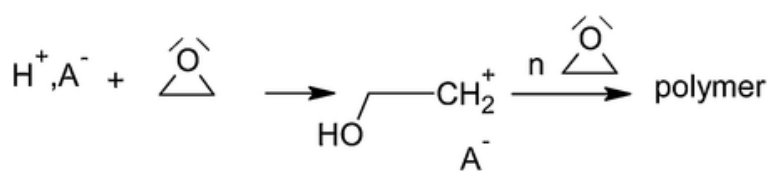

Scheme 20. Reaction of initiating cations (here $\mathrm{H}^{+}$) with cationic polymerization monomers (here oxirane) and subsequent cationic propagation.

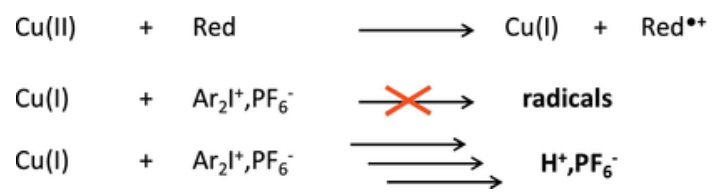

scheme 21. Reactions involved in $\mathrm{Cu}(\mathrm{II}) /$ Reducing agent (Red)/iodonium salt (here $\mathrm{Ar}_{2} \mathrm{I}^{+}$with $\mathrm{PF}_{6}^{-}$counter anion) $\mathrm{RCP}$ initating systems. 
erated in situ matters: it is considerably lower for the acetate, benzoate or octoate derivatives (e.g. for acetate $\mathrm{E}_{\mathrm{ox}}=-0.6 \mathrm{~V} v s \mathrm{SCE}$ [173]) than for more recent amino or phosphino substituted $\mathrm{Cu}(\mathrm{I})\left(\mathrm{E}_{\mathrm{ox}}\right.$ ranging from 0.6 to $1.3 \mathrm{~V} v s \mathrm{SCE}[174,175])$ that were strictly inactive in the redox decomposition of iodonium salts (no light, RT). This fact confirms that $\mathrm{Cu}(\mathrm{I})$ reduces the iodonium salt.

$\mathrm{Cu} /$ reducing agent/iodonium salt reactions were applied to several RCP initiations as shortly reviewed in Table 2 (1-5). Generally speaking, the easiest RCP were obtained using iodonium salt and activated epoxides. On the contrary, for s-trioxane RCP, use of heat was required as the propagating constants are more limited for that monomer [170]. Interestingly, the study by Yagci et al. proposed pyridinium salts instead of iodonium salts in combination with $\mathrm{Cu}(\mathrm{II}) / \mathrm{VitC}$ derivatives [176]. Interesting correlations between the reduction potential of the oxidizing agent and the rate of polymerization were found; nevertheless, the performances remained limited in terms of conversions and kinetics.

In parallel, many research groups have shown that the free radicals generated by thermolytic [177,178] or by photolytic [179-183] methods can be oxidized by diaryliodonium salts, and in some other examples by sulfonium or pyridinium salts, generating superacids species. This strategy is commonly referred as the free radical promoted cationic polymerization (FRPCP) [184,185]. Garra et al. proposed a RCP initiating strategy (still using $\mathrm{Cu}$ based chemistry) inspired by FRPCP as illustrated in Scheme 22 [171]. The acetylacetonate radicals (and other potential radicals arising from the iodonium decomposition and as well as hydrogen abstractions) can be oxidized in order to form highly reactive cations. One issue in that system and in the adaptation of many RFRP initiating systems to FRPCP is that the phosphines or more generally the (redox) reducing agents bear strong lone pair that tends to inhibit CP propagation. Therefore, the performances remained limited. Another study by Yilmaz et al. pro- posed a $\mathrm{Cu} / \mathrm{VitC} / \mathrm{BPO}$ radical initiating system that can lead to cations using a newly synthesized sulfonium salt [186]; it is a promising introduction to new categories of RCP initiating systems.

\subsection{Other catalysts}

Other RCP initiating paths not based on Cu-based chemistry are also reported in the litterature (Table 3). Many of them use noble metals. The use of noble metals is sometimes seen as an "out to date" strategy in inorganic catalysis; nevertheless, the amount of $\mathrm{Pt}$ and $\mathrm{Rh}$ catalysts used by Crivello et al.in RCP remained very limited, typically a few ppm (0.5-8 ppm) [23]. Especially, in Scheme 23 (Table 2, entries 1-4), the decomposition of iodonium salts by a silane injected in the reaction medium by the vapor phase leads to an outstandingly efficient RCP initiation: it was also compatible with oxetanes and non activated $\mathrm{CP}$.

A few studies also considered sulfonium salts instead of iodonium salts in RCP. Indeed, it is more difficult to reduce triarylsulfonium salts $\left(\mathrm{E}_{\mathrm{red}}=-28 \mathrm{kcal} / \mathrm{mol}\right)$ [188] than diaryliodonium salts $\left(\mathrm{E}_{\mathrm{red}}=-5 \mathrm{kcal} / \mathrm{mol}\right)$ [187]. One was successful using noble metal catalysts and vapor state silane for the polymerization of highly reactive activated epoxides and vinyl ethers [189]. These monomers show a better reactivity compared to classical epoxide ones (see below). Acylsulfonium salts are also highly efficient compared to iodonium salts as shown by Molleo et al. [190] In that study, an interesting tuning of the gel time can be performed through the adjustment of the ppm level of metallic catalyst (e.g. $30 \mathrm{~s} \mathrm{~g}$ th ne to $110 \mathrm{~s}$ when changing the Pt catalyst level from 8 to $2 \mathrm{ppm}$ ). be proposed though a difficult RCP of oxetanes was observed [191] when highly successful for activated epoxides and vinylethers.

Next, dialkyl borane 9-BBN does not require any noble metal catalysts and proceeds via a direct and efficient reduction of the iodo-

Table 2

Summary of RCP initiating systems involving Cu-based systems.

\begin{tabular}{|c|c|c|c|c|c|c|}
\hline entry & Cu complex & Reducing agents & Oxidizing agents & RCP Monomers & Temp. & Ref. \\
\hline 1 & $\mathrm{Cu}(\mathrm{II})$ benzoate & $\operatorname{Tin}(\mathrm{II})$ & Diaryliodonium salts & In solvent: epoxides / oxetanes /s-trioxane & $\begin{array}{l}40^{\circ} \mathrm{C} \text { and } \\
45^{\circ} \mathrm{C}\end{array}$ & [171] \\
\hline 2 & $\mathrm{Cu}(\mathrm{II})$ benzoate & VitC & Diaryliodonium salts & $\begin{array}{l}\text { In solvent: activated epoxide }(\mathrm{CHO})\left(25^{\circ} \mathrm{C}\right) / \mathrm{s}- \\
\text { trioxane }\left(50^{\circ} \mathrm{C}\right)\end{array}$ & $\begin{array}{l}25^{\circ} \mathrm{C} \text { and } \\
50^{\circ} \mathrm{C}\end{array}$ & [170] \\
\hline 3 & $\mathrm{Cu}(\mathrm{I})$ benzoate & - & Diaryliodonium salts & In solvent: activated epoxide (CHO) & $25^{\circ} \mathrm{C}$ & [187] \\
\hline 4 & $\mathrm{Cu}$ (II) benzoate & benzoins & Diaryliodonium salts & $\begin{array}{l}\text { In solvent: activated epoxide }(\mathrm{CHO})\left(25^{\circ} \mathrm{C}\right) / \\
\text { epoxides }\left(45^{\circ} \mathrm{C}\right)\end{array}$ & $\begin{array}{l}25^{\circ} \mathrm{C} \text { and } \\
45^{\circ} \mathrm{C}\end{array}$ & [187] \\
\hline 5 & $\begin{array}{l}\mathrm{Cu}(\mathrm{II}) \\
\text { 2-ethylhexanoate }\end{array}$ & Tin(II) or VitC & Diaryliodonium salts & In bulk: activated epoxides (EPOX) & $\mathrm{RT}\left(23^{\circ} \mathrm{C}\right)$ & [172] \\
\hline 6 & $\mathrm{Cu}(\mathrm{II})$ benzoate & VitC derivative & $\begin{array}{l}\text { Pyridinium and allyl sulfonium } \\
\text { salts }\end{array}$ & In solvent: activated epoxide (CHO) & $25^{\circ} \mathrm{C}$ & [176] \\
\hline 7 & $\mathrm{Cu}(\mathrm{II})$ benzoate & VitC & $\mathrm{BPO}+$ allyl sulfonium salt & In bulk: activated epoxide (CHO) & RT & [186] \\
\hline 8 & $\mathrm{Cu}(\mathrm{II})$ acetylacetonate & $\begin{array}{l}\text { phosphine } \\
\text { (2dppba) }\end{array}$ & Diaryliodonium salts & In bulk: activated epoxides (EPOX) & $\mathrm{RT}\left(23^{\circ} \mathrm{C}\right)$ & [172] \\
\hline
\end{tabular}

Tin(II): Tin 2-ethylhexanoate; VitC: ascorbicacid; CHO: cyclohexeneepoxide; 2dppba: 2-diphenylphosphino benzoicacid.

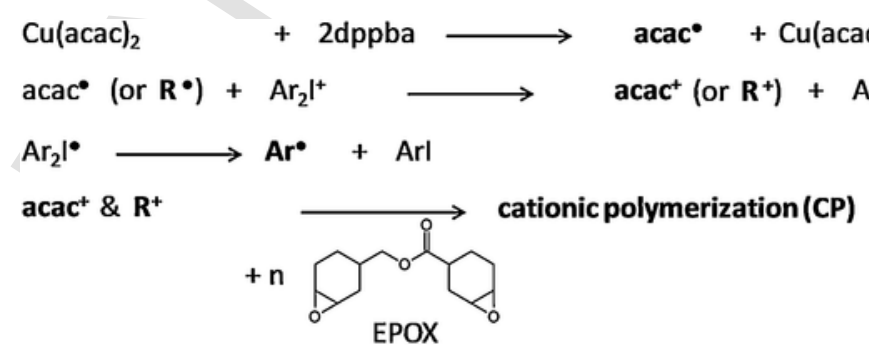

(r1)

(r2)

(r3)

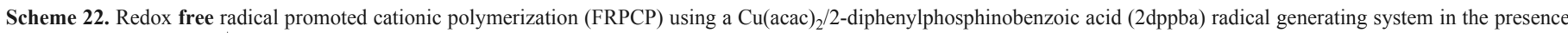
of an iodonium salt $\left(\mathrm{Ar}_{2} \mathrm{I}^{+}\right)$[173]. Copyright 2018. Adapted with permission from the American Chemical Society. 
Table 3

Summary of RCP initiating systems not involving $\mathrm{Cu}$.

\begin{tabular}{|c|c|c|c|c|c|c|}
\hline Entry & $\begin{array}{l}\text { Metal catalyst (ppm } \\
\text { level) }\end{array}$ & Reducing agent & Oxidizing agents & RCP Monomers & Temp. & Ref. \\
\hline 1 & $\begin{array}{l}{\left[\mathrm{Cl}(\mathrm{CO})_{2} \mathrm{Rh}(\mathrm{I})\right]_{2} /} \\
\text { Lamoreaux's }(\mathrm{Pt}) \\
\text { catalyst }\end{array}$ & $\begin{array}{l}\text { Vapour state } \\
\text { silanes }\end{array}$ & $\begin{array}{l}\text { Diaryliodonium salts }\left(\mathrm{A}^{-}=\mathrm{SbF}_{6}^{-}\right. \\
\left.\mathrm{PF}_{6}^{-} ; \mathrm{AsF}_{6}^{-} ; \mathrm{B}\left(\mathrm{C}_{6} \mathrm{~F}_{5}\right)_{4^{-}}\right)\end{array}$ & $\begin{array}{l}\text { In bulk: epoxides / activated } \\
\text { epoxides / oxetanes / } \\
\text { vinylethers }\end{array}$ & RT & {$[23]$} \\
\hline 2 & $\begin{array}{l}\mathrm{Rh}(\mathrm{I}) / \mathrm{Rh}(\mathrm{II}) / \mathrm{Pd}(\mathrm{II}) / \\
\text { Karstedt's (Pt) catalyst }\end{array}$ & $\begin{array}{l}\text { Vapour state } \\
\text { silanes }\end{array}$ & Sulfonium salts & $\begin{array}{l}\text { In bulk: activated epoxide } \\
\text { (CHO) / vinylethers }\end{array}$ & RT & [189] \\
\hline 3 & $\begin{array}{l}\text { Lamoreaux's }(\mathrm{Pt}) \\
\text { catalyst }\end{array}$ & $\begin{array}{l}\text { Vapour state } \\
\text { silanes }\end{array}$ & $1 \overline{=}$ HeteroaromaticOnium Salts & In bulk: activated epoxides & RT & {$[191]$} \\
\hline 4 & $\begin{array}{l}\text { Karstedt' (Pt) catalyst / } \\
\mathrm{Cl}_{2}(\mathrm{COD}) \mathrm{Pd}(\mathrm{II})\end{array}$ & $\begin{array}{l}\text { Vapour state } \\
\text { silanes }\end{array}$ & Dialkylphenacylsulfonium Salts & $\begin{array}{l}\text { In bulk: activated epoxides, } \\
\text { oxetanes, vinyl ethers }\end{array}$ & RT & [190] \\
\hline 5 & None & 9-BBN & Diaryliodonium salts & $\begin{array}{l}\text { In bulk: epoxides / activated } \\
\text { epoxides / oxetanes / } \\
\text { vinylethers }\end{array}$ & RT & [194] \\
\hline 6 & None & $\mathrm{Fe}(0)$ (surface) & $\operatorname{Ag}(\mathrm{I})$ salt $\left(\mathrm{AgSbF}_{6}\right)$ & $\begin{array}{l}\text { In bulk: activated epoxides } \\
\text { (EPOX) }\end{array}$ & RT (hours) & [192] \\
\hline 7 & None & Silane & $\operatorname{Ag}(\mathrm{I})$ salt $\left(\mathrm{AgSbF}_{6}\right)$ & $\begin{array}{l}\text { In bulk: activated epoxides } \\
\text { (EPOX) / vinyl ethers }\end{array}$ & RT & [195] \\
\hline 8 & None & $\begin{array}{l}\text { Silane and } \\
10 \mathrm{wt} \% \text { alcohol } \\
\text { (starch) }\end{array}$ & $\operatorname{Ag}(\mathrm{I})$ salt $\left(\mathrm{AgSbF}_{6}\right)$ & In bulk: $\varepsilon$-caprolactam & RT (hours) & [193] \\
\hline 9 & None & $\mathrm{H}_{2} \mathrm{O}$ & $\begin{array}{c}\mathrm{KSbF}_{6} \text { and } \mathrm{NaSbF}_{6} \\
=\end{array}$ & $\begin{array}{l}\text { Bulk: epoxides / activated } \\
\text { epoxides (CHO, EPOX) }\end{array}$ & $\begin{array}{l}\text { RT }(2 \mathrm{~h}) \text {, conversion limited } \\
\text { for epoxides }(30 \%)>90 \% \\
\text { for activated ones }\end{array}$ & [196] \\
\hline
\end{tabular}

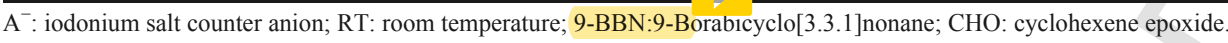
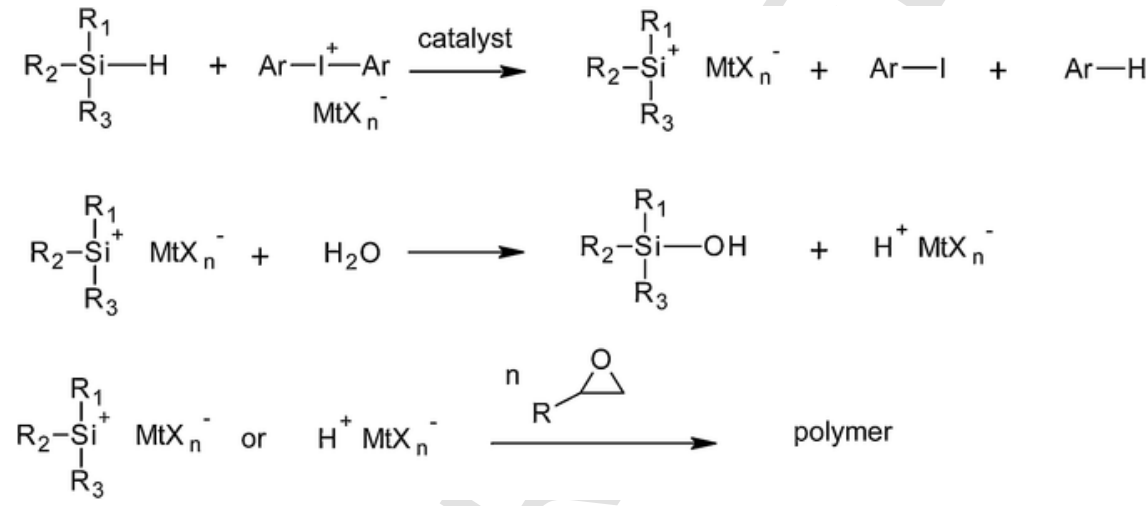

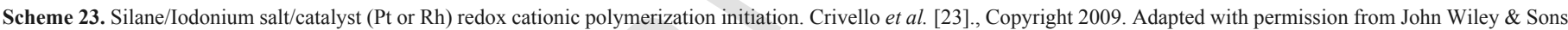
Inc.

nium salt (entry 5) In a more exotic way, ferrocenium (III) was proposed as initiat 1 rg he RCP of EPOX from a surface but the reaction was found quite slow (several hours) [192]. Another option is to use $\operatorname{Ag}(\mathrm{I}) /$ Silane reaction to produce cations. $\operatorname{Ag}(\mathrm{I}) /$ silane mechanism will be presented below (5.2) when integrated in a redox dual FRP/CP initiation. $\operatorname{Ag}(\mathrm{I})$ salt was applied to the $\varepsilon$-caprolactam $\mathrm{CP}$ in the presence of silane and $10 \%$ alcohol (from starch) as a protogenic specie [193]; the polymerization efficiency remained limited (13 $\mathrm{h}$ to reach tack-free polymers) but interesting features exist in these systems such as the in situ generation of $\operatorname{Ag}(0)$ nanoparticles.

Finally, $\mathrm{Ni}(\mathrm{II}), \mathrm{Ag}(\mathrm{I}), \mathrm{Mn}(\mathrm{III}), \mathrm{Co}(\mathrm{II})$ and $\mathrm{Fe}(\mathrm{II})$ metals were essentially found inactive in RCP with benzoin and/or VitC and Iodonium salts $[170,187]$ so as $\operatorname{Ir}(\mathrm{I})$ and $\mathrm{Ru}(\mathrm{II})$ in the presence of sulfonium salts and silanes [189]. Noteworthy, metal catalysts should avoid bearing phosphine and amine ligands as it was proposed that these ligands inhibit CP propagation [23].

\subsection{Monomers proposed in redox $C P$}

RCP is an excellent way to induce the curing of epoxy resins under mild conditions (RT) with almost no energy consumption as commented by Green and Wittcoff [196]. Generally speaking, monomers compatibles with RCP (Scheme 24) can be chosen among vinylethers (or $N$-vinyl carbazole), oxetanes, epoxides or activated epoxides. For these latter, the cyclohexane ring under epoxide moiety leads to a

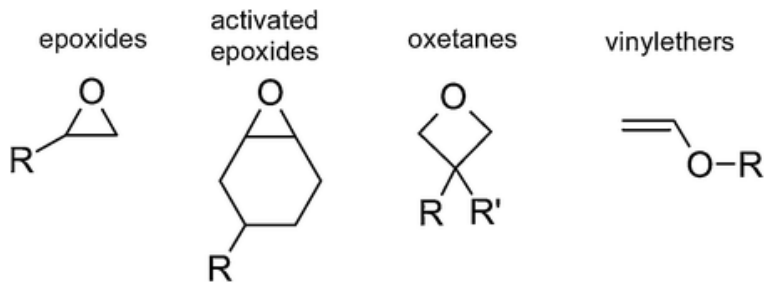


tenser epoxide ring thus its opening is favored. Such monomers are said to be "activated" (i.e. more reactive). s-trioxane was also proposed in a few studies [171]. One study also proposed the use of tetrahydrofuran as a potential monomer for RCP but the performances remained very low with a conversion of less than $10 \%$ after one hour [170]. Finally, $\varepsilon$-caprolactam was efficiently polymerized under heated medium $\left(60^{\circ} \mathrm{C}\right)$ using $\mathrm{Cu}(\mathrm{II}) /$ benzoin/aryl iodonium RCP initiating system [187]. Noteworthy, the examples given in Scheme 24 show one function that can be polymerized and lead to linear polymers without crosslinking between macromolecules [198]. In many industrial applications (e.g. adhesives [199]), multifunctional monomers are used for example (3,4-epoxycyclohexane) methyl 3,4-epoxycyclohexylcarboxylate (or EPOX) activated epoxide. They also result in polymers with high glass transition temperatures $\left(\mathrm{T}_{\mathrm{g}}>130^{\circ} \mathrm{C}\right)[192]$; nevertheless, they are still relatively expensive compared to FRP monomers. The adhesive properties of epoxides to many substrates, the low shrinkage and the absence of oxygen inhibition encourage the development of novel multifunctional monomers compatible with $\mathrm{CP}$ $[183,200]$.

Finally, too high amounts of water or other nucleophile compounds tend to inhibit the $\mathrm{CP}$ propagation. On the contrary, at reduced amounts $(<1 \mathrm{wt} \%)$, water or other protogens (e.g. alcohols [201]) can be useful as co-catalysts to enhance the cationic ring-opening polymerization [202].

\section{Redox agents in controlled polymerizations}

\subsection{Differences between controlled and conventional polymerization}

During these last 20 years, the controlled polymerizations and its subsequent developments correspond to a very dynamic field in polymer science [203,204]. It is therefore important to mention it in the present review where mainly uncontrolled (conventional) polymerization are presented. A few differences between controlled and conventional polymerization are outlined in Scheme 25: free radical polymerization (FRP) is opposed to controlled/liv ng adical polymerization (CRP). Obviously, the goal of that Scheimi is to sketch a general trend. CRP and FRP involve similar initiating reactions. In FRP, the propagation is extremely fast (high $\mathrm{k}_{\mathrm{p}}$ ) and the quenching leads irreversibly to inactive species (inhibitions, recombinations, terminations...). On the opposite, most of the macromolecules in CRP are in a latent state $\left(\mathrm{R}(\mathrm{M})_{\mathrm{n}}-\mathrm{X}\right)$ that can be re-opened using activating

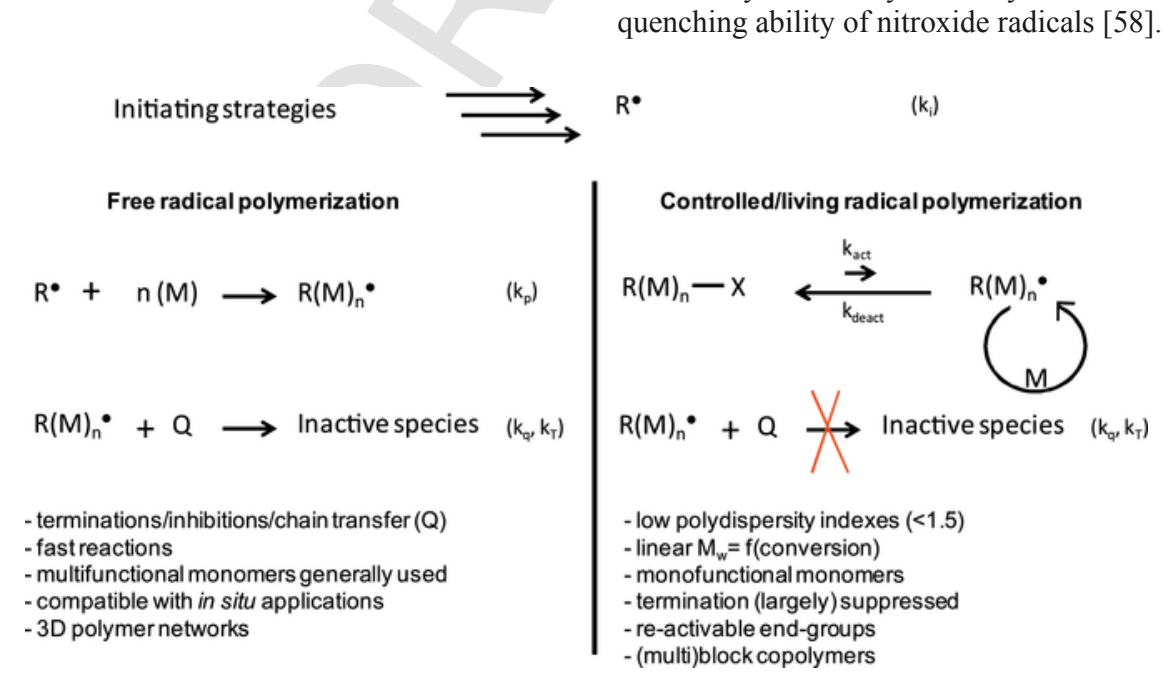

chemical mechanisms. The deactivation process is a very fast reaction so that the (irreversible) termination reactions are largely suppressed in CRP (deactivation is faster than inhibitions).

Next, fast polymerization kinetics in uncontrolled FRP are largely related to the initiating strategies and very fast reactions: below seconds in photo FRP to second-minutes time range in RFRP. Adversely, $\mathrm{CRP}$ are less rapid reactions but the final polymers can have controlled molecular weights. When the polymer scientist's imagination can lead to outstandingly controlled architecture in CRP (stars, brushes, linear polymers, strain-adaptive elastomers [205]...), the precise control of the final polymer structure in FRP is less important but dense 3D polymer networks can be obtained particularly using multifunctional monomers. FRP is compatible with plenty of in situ applications i.e. the synthesis of the polymer is performed directly where curing was required: this is true for adhesives, composites, $3 \mathrm{D}$ printing and lithographic techniques, chemical anchoring systems....Some very recent researches by the group of Asua also showed that control in the polymerization chain growth results in a more homogeneous crystallization of the final acrylic polymer as observed in wide angle/small angle (WAXS/SAXS) diffraction experiments [206]. Noteworthy, there is some counter-examples to the general Scheme 25: FRP of homopolymers [139], recent efforts for the introduction of multifunctional monomers in CRP [198], extremely fast (20 min) reversible-deactivation radical polymerizations (RDRPs) recently developed... [207].

Interestingly, reducing and oxidizing agents found in conventional FRP and CP initiating systems are also found in the literature devoted to the controlled polymerization. We will therefore examine a few examples of reducing and oxidizing agents that are found in redox reactions applied to the controlled polymerization. We will not review external sources of electrons (see few examples suggested) such as photocontrolled polymerizations [207-211], electrochemically mediated controlled polymerizations [212-217] or mechanoCRP [30,218,219] as these setups imply a deviation from the initial scope of the present review (no energy consumption). In reversible-deactivation radical polymerizations (RDRPs), redox agents are also used: i) for the first generation of radicals or ii) to accelerate kinetics of either deactivation or activation processes especially, for that last point, in atom transfer radical polymerization (ATRP) and reversible addition-fragmentation chain transfer (RAFT) [220]. Less examples are found in nitroxide-mediated polymerization (NMP) as the carbon to oxygen bond was mostly cleaved by thermolysis and reformed thanks to the radical quenching ability of nitroxide radicals [58].

Scheme 25. Major and most commonly encountered differences between FRP and controlled/living radical polymerizations. 


\subsection{Examples of reducing agents in controlled polymerization}

As outlined by Fantin et al., the electron transfer reactions are of huge importance for the determination of ATRP kinetics [221]. Particularly, reducing agents can enhance the CRP kinetics. This is especially true for activators regenerated by electron transfer (ARGET) ATRP [222,223]. For example, as in RFRP or RCP, addition of VitC as a reducing agent in CRP allows to outstandingly enhance the polymerization kinetics (Scheme 26) [224]. In RAFT polymerization $[225,226]$, the group of Boyer et al. also showed that reducing agents (e.g. VitC) can also enhance the CRP and allow the synthesis to be carried out under mild aerated conditions [227,228].

Similarly, the direct introduction of reducing agents such as metallic wires $(\mathrm{Cu}(0)$ or $\mathrm{Ag}(0)$ wires ) [229-234] allows to outstandingly enhance the CRP kinetics through the regeneration of ligand/ $\mathrm{Cu}(\mathrm{I}) \mathrm{ac}-$ tivators. Again, a parallel can be found with the conventional redox polymerizations: raw metal (e.g. $\mathrm{Fe}(0))$ is also useful as a strong reducing agent in RCP [193]. The library of reducing agents in single electron transfer living radical polymerization (SET-LRP) also include phenols [235], amines [236], Tin(II) [237], hydrazine... [238] which are also present in conventional polymerization [55].

\subsection{Examples of oxidizing agents in controlled polymerization}

A recent advanced work by Fors et al. proposed the participation of the Ferrocenium oxidizing agent as a chemical stimulus to oxidize radicals into cations (Scheme 27) [239]. This lead to on-demand dual cationic/radical polymerizations that were applied to vinylether/ acrylate copolymerization. Ferrocene and ferrocenium chemistry is also largely present in conventional redox polymerizations $[64,67,69,192,240]$. In both modes (controlled or conventional), ferrocene-based redox agents enable outstanding accelerating effects and reactions under mild conditions.

More generally, many compounds can be defined as oxidizing agents in controlled polymerization. Oxidizing agents are compounds that can undergo a reduction reaction; therefore this category can include all the $\mathrm{Cu}(\mathrm{II})$ family in the ATRP-like mechanisms, Co(III), [204,241], alkyl halides, peroxides.... These types of derivatives are classical compounds found in RFRP and RCP.

\section{Integration of redox polymerization in dual-curing systems}

A dual-curing process can define the combination of two curing processes occurring sequentially or simultaneously. Some authors go further [242] describing it as: "a method to combine two otherwise distinct polymer networks" but this definition is a restriction that is closer to Interpenetrating Polymer Networks (IPN) generation. It does not include the dual curing processes where the same monomers are used but the radical generation is performed through two different chemical mechanisms e.g. photo/thermal dual curing [6,243-249].

The goal of combining two curing processes is to improve the materials that would have been obtained by using only one of this curing approach. Several cross-linking reactions can be combined for example thiol-acrylates, thiol-epoxy, thiol-ene, Diels-Alder, Michael addition, isocyanate-acrylate, epoxy cationic polymerization... [250] As in RFRP or RCP, redox initiation of thiol-ene reaction has the advantage of unlimited depth of cure compared to the thermal or photo initiated reactions [251]. Here, in order to reduce the size of the manuscript, we will only review one example where two different mechanisms (photo and redox) induce the curing of the same resin and two examples where a IPN is generated from a redox dual CP/FRP. More references can be found in the literature [242,250,252].

\subsection{Redox photoactivated polymerization (FRP)}

A hybrid strategy between photopolymerization (Scheme 28) and redox polymerization can be proposed and was referred as a redox photoactivated polymerization. Such systems are also sometimes referred as dual-curing systems but we preferred redox photoactivated systems as being much more specific (see vide supra). In redox photoactivated polymerization, the light activation of redox systems thanks to PIS is interesting since: i) the efficiency of the PIS should highly increase the surface conversion (where the light absorption is maximum); ii) an acceleration of the slow redox process is possible on-demand and iii) the global conversion can be improved (more active species generated). Additionally, in some specific cases, photoactivation enhanced the chemical bleaching which is of importance for dental cements [253].

In dental materials, Mehmood et al. studied dual-curing systems based on redox and photo FRP [254]. Most (5 out of 10) of the flexural strengths for the final composites were highly enhanced by the light activation compared to a pure redox or photo curing system taken separately (mild improvements for the 5 other composites).

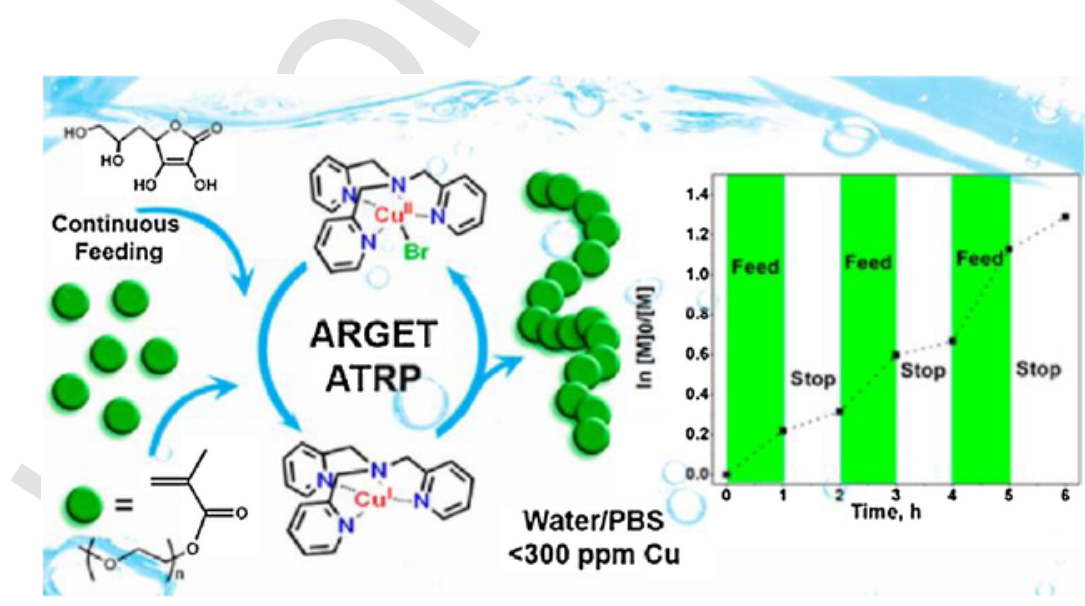

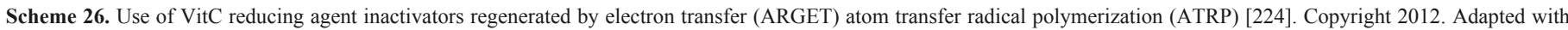
permission from the American Chemical Society. 


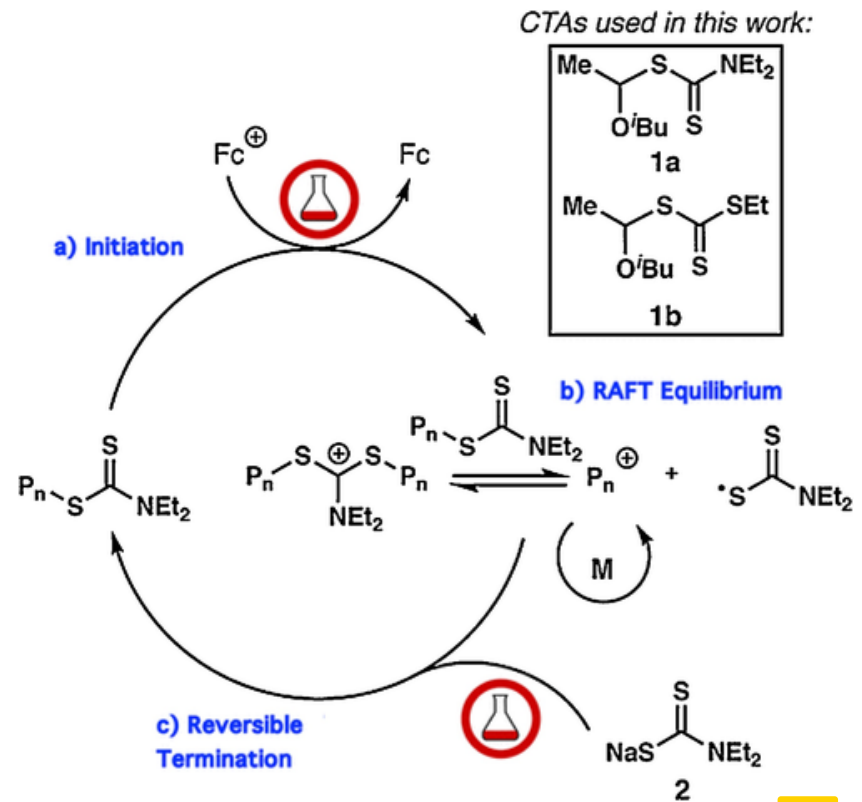

Scheme 27. Fors and coworkers orthogonal chemical stimulus using ferriciniun uxıdizing agents in controlled cationic polymerization [239]. Copyright 2018. Adapted with permission from the American Chemical Society.

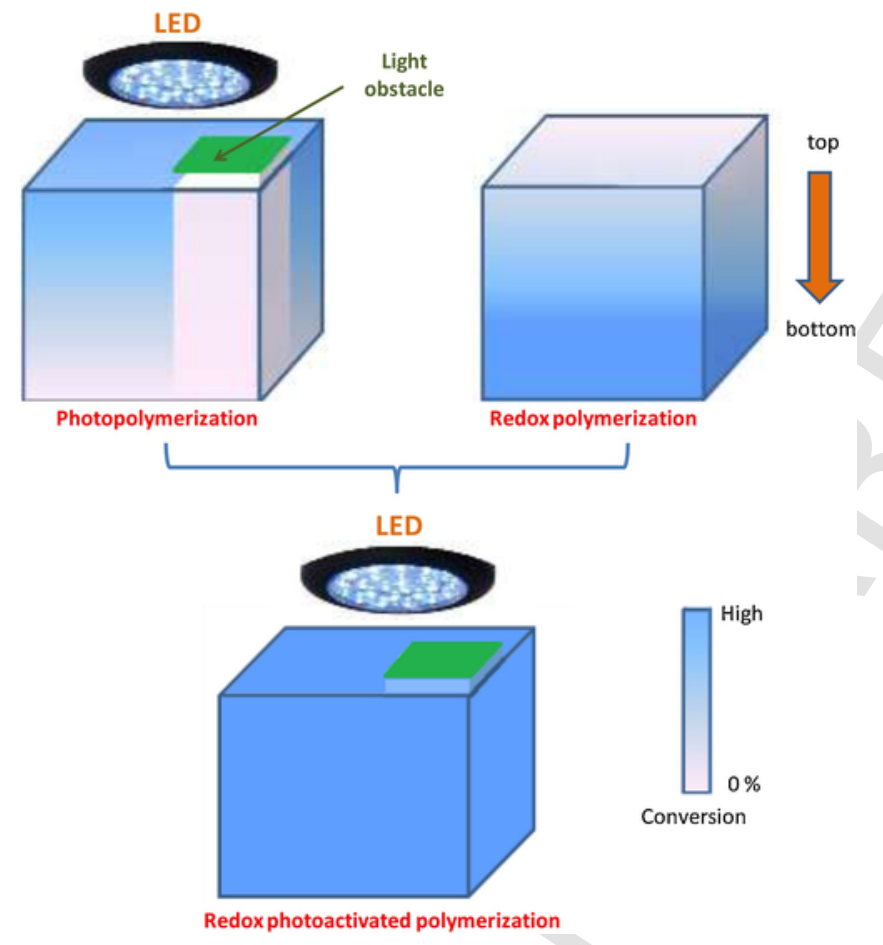

Scheme 28. Main features of Photopolymerization, Redox photoactivated polymerization and Redox polymerization under air [52]. Copyright 2016. Adapted with permission from the American Chemical Society.

Most of the literature shows similar conclusions: light activations in dual curing systems have generally positive effects on the dental material properties [255-257].

The possibility to generate thick samples in the dual curing of acrylic resins (20 mm thick, $50 \mathrm{~g}$ ) was also demonstrated [258].
Diphenyl(2,4,6-trimethylbenzoyl)phosphine oxide (TPO) was the PIS when Cobalt/peroxide system was used as a RFRP initiating system. The system exhibits a mild exothermicity of $100{ }^{\circ} \mathrm{C}$ after $150 \mathrm{~min}$ without irradiation. Under UV $\left(420 \mathrm{~mW} / \mathrm{cm}^{2}\right)$ light irradiation, a more efficient polymerization $\left(210^{\circ} \mathrm{C}\right)$ was possible after $35 \mathrm{~min}$.

The dual curing examples presented in Fig. 3 illustrate what can be expected from redox photoactivated FRP initiations: Accelerated gel times (Fig. 3A and C), enhanced surface curing (Fig. 3B), enhanced global curing (conversion, Fig. 3A, C and D) and chemical bleaching (Fig. 3D).

Redox photoactivated dual-curing are very promising but the effect of the light activation can be mild to extremely efficient according to: i) the PIS, ii) the redox initiating systems and iii) the characteristics (thicknesses) of the polymerizable media. First, the PIS/redox systems must be compatible for storage in two separated components; second, redox polymerization systems must have a relatively long gel time and, finally, light penetration during light activation is not optimum throughout the entire polymerizable medium which results in variable light activation improvements.

Therefore, there is a huge need to i) develop systems capable of redox photoactivated polymerization and ii) study photoactivation effect on the polymerization efficiency (on-demand acceleration, surface curing and global conversion enhancements).

\subsection{Dual-curing: redox FRP/CP}

In this part, we will explore the possibility of generating interpenetrating networks (IPNs) from the simultaneous redox FRP and redox CP initiation of (meth)acrylates and (activated) epoxides, respectively. When orthogonal controlled radical/cationic polymerizations are rapidly emerging as a smart way to produce copolymers [239,259-261], bulk dual RFRP/RCP are not easily encountered in the literature. As many IPN generating strategies, dual FRP/CP should show great advantages of both propagating modes and reduced oxygen inhibition: a smart combination of both networks is expected. Two studies can be mentioned concerning the dual RFRP/RCP initiation: first, the pioneering work by Tehfe et al., inspired by recent advances in the light induced dual FRP/CP, used Diphenylsilane (DPSi) in combination with $N$-vinylcarbazole (FRPCP enhancing additive) with $\mathrm{AgSbF}_{6}$ salt [262]. The proposed chemical mechanisms are highlighted in Scheme 29: the silane is reduced by $\operatorname{Ag}(\mathrm{I})$ which leads to the formation of a silyl radical (useful for RFRP of acrylates) and $\mathrm{H}^{+}, \mathrm{SbF}_{6}{ }^{-}$superacids that are useful for (activated) epoxides RCP initiation. According to the concentration of silver salt, an oxidation of the radicals can also be proposed leading to other superacids (RCP initiating ones).

As a second example, the very recent work of Garra et al. which proposed another strategy that combines a $\mathrm{Cu}(\mathrm{II}) /$ reducing agent (VitC or tin octoate)/Onium salt RCP initiating system with a recently developed $\mathrm{Cu}(\mathrm{II}) /$ reducing agent/peroxide RFRP initiating system. The proposed catalytic cycles are gathered in the Fig. 4 . Synergistic $\mathrm{C}=\mathrm{C}$ and epoxide conversions $v s$. time are observed in Fig. 4B. Very high final reactive function conversions (for multifunctional monomers) were observed at the end of the dual-curing. Interestingly, dual RFRP/RCP results in a much more improved $\mathrm{C}=\mathrm{C}$ curing at the surface compare to the reference RFRP initiating system (4- $N, N$ TMA/BPO) and to the $\mathrm{Cu}$ (II)/VitC/BPO RFRP system developed (Fig. 4C). The usefulness of dual RFRP/RCP was clearly demonstrated: that IPN allows a faster increase of the viscosity (RCP network) at the surface that prevents oxygen diffusion; the oxygen inhibited layer will therefore be clearly reduced. 

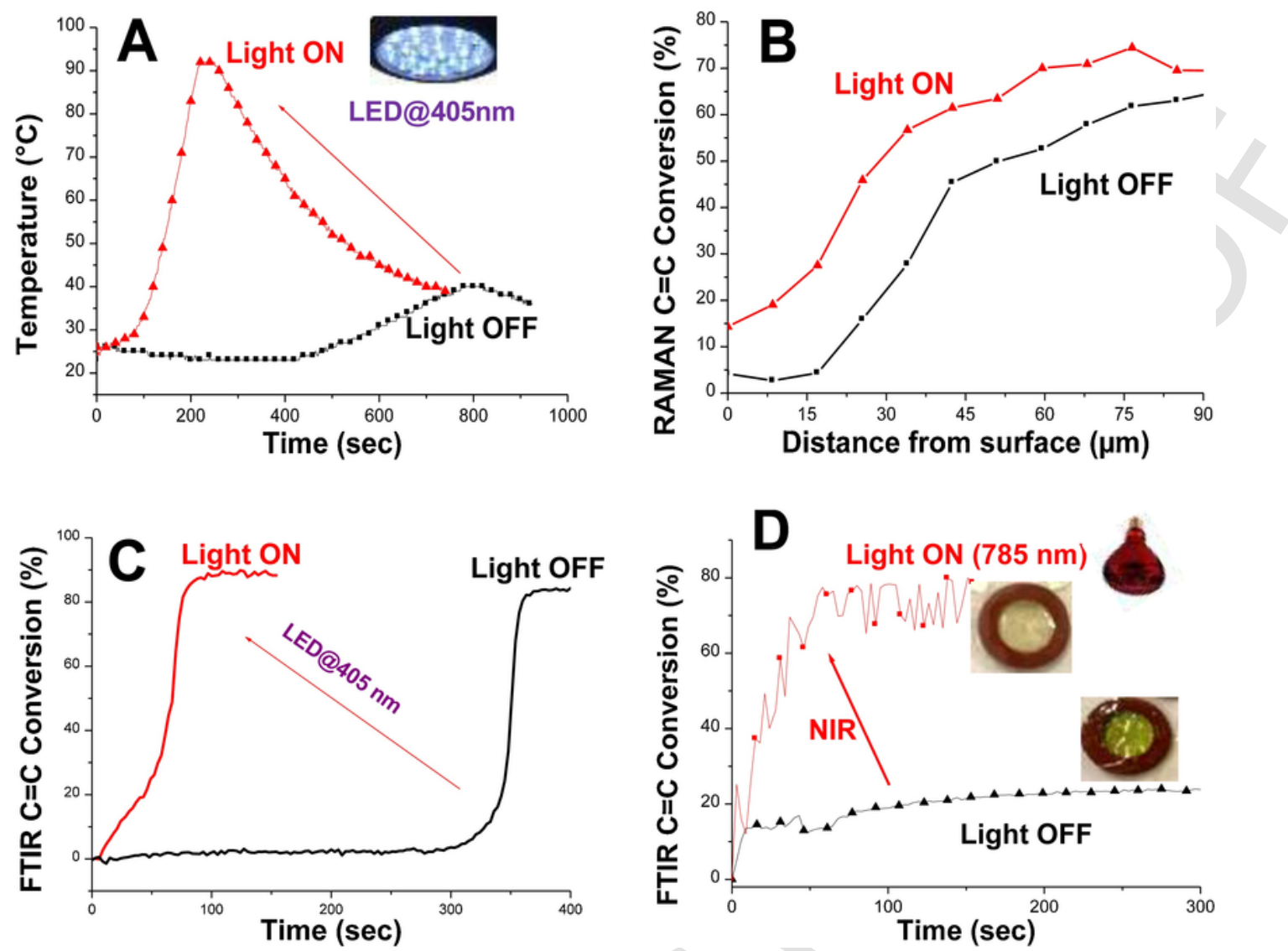

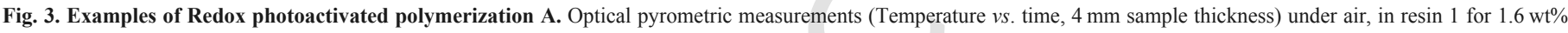

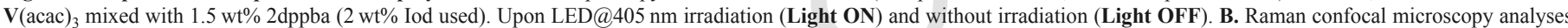

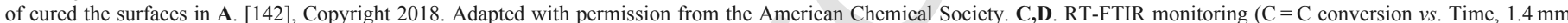

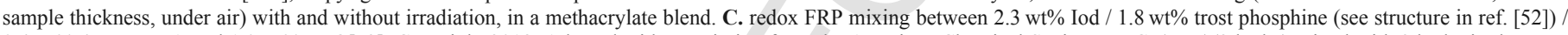

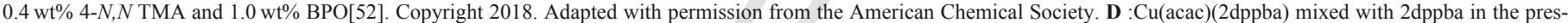
ence of tempol [254]. Copyright 2017. Adapted with permission from John Wiley \& Sons Inc.

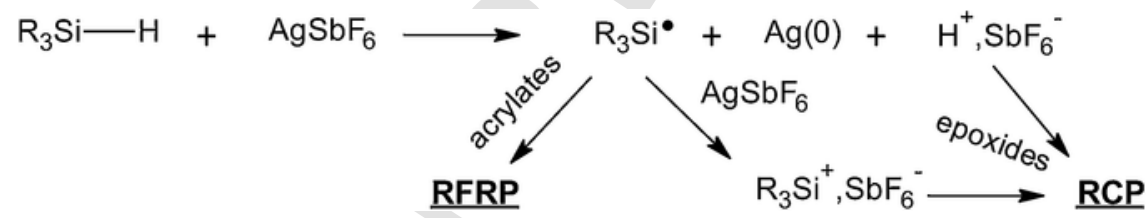

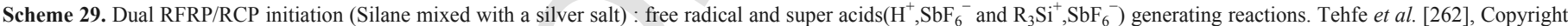
2015. Adapted with permission from MDPI.

In the same study, a $\mathrm{Cu}(\mathrm{acac})_{2} /$ phosphine (2dppba)/Iodonium salt system was also successful to initiate dual FRP/CP but with mild performances due to the phosphine inhibition of cationic propagation.

\section{Applications}

\subsection{Redox polymerization in bulk}

Redox polymerization of clear/bulk/neat resins is reviewed in the literature $[263,264]$. The properties of the used monomer will be a key feature for the final applications. High degree of crosslinking in acrylates will lead to dense polymer network with high glass transition temperatures. On the contrary, choosing specific acrylamides can lead to interesting gel polymerizations initiated by ascorbic acid/ferrous sulfate/ammonium persulfate in the literature [265]. Hydrogels are also accessible via redox polymerization [266-271]. In the case of a redox polymerization under the mild conditions defined above, strong limitations could be identified for the efficient commercially available RFRP initiating systems:

1) Toxicity/instability/hazard of some of the oxidizing agents (e.g. peroxides);

2) Carcinogenicity of the reducing agents (e.g. N-aromatic amines);

3) Very weak curing of the oxygen inhibited surfaces (tacky polymers, e.g. $>70 \mu \mathrm{m}$ inhibited layer for $4-N, N$ TMA/BPO redox system [52])) $[40,41,225,272]$;

4) Absence of acceleration/slow down control of the polymerization after the 2-component mixing.

5) Mechanical properties of the redox polymer can sometimes be improved through higher conversions [254,257]. 

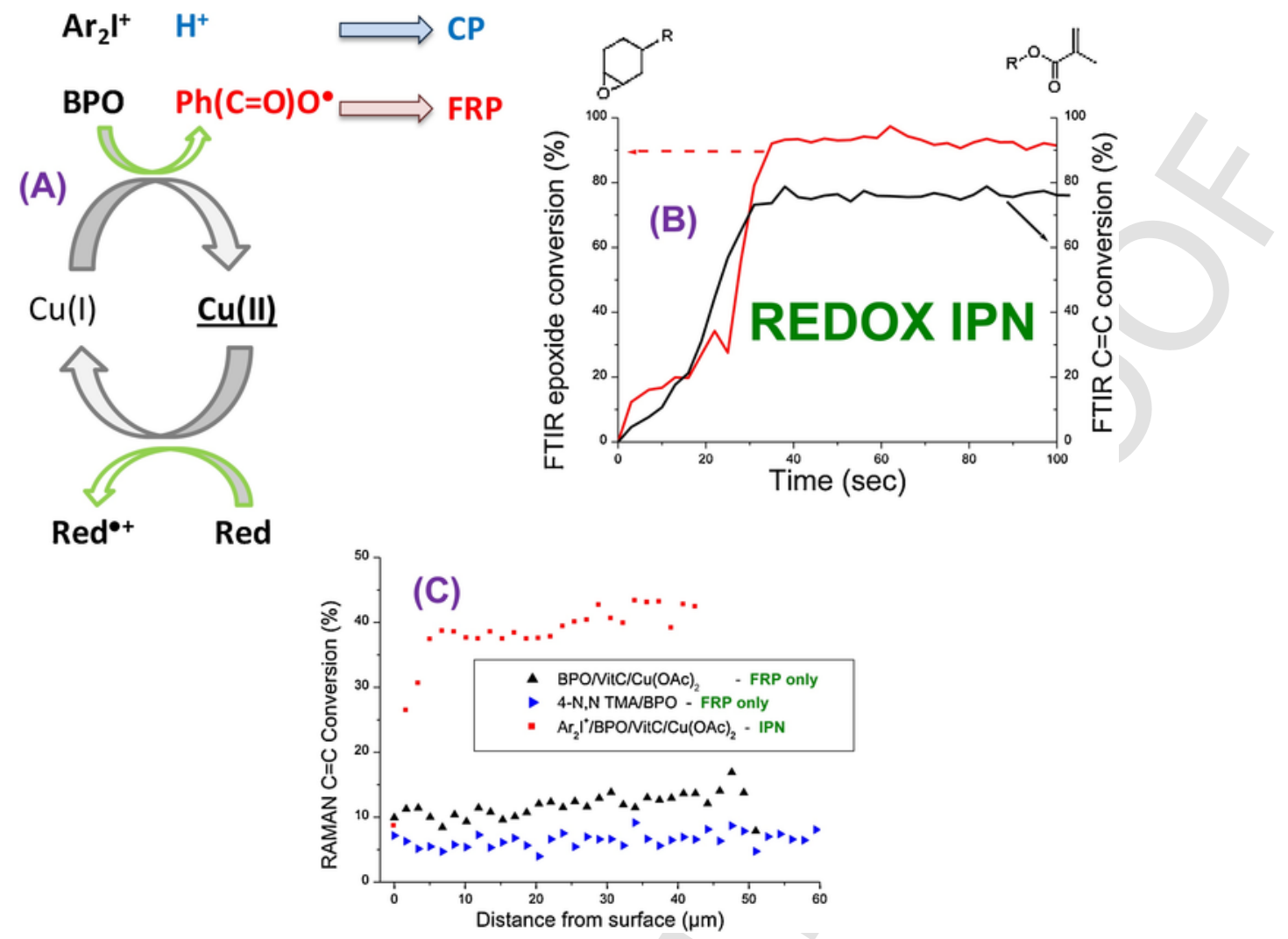

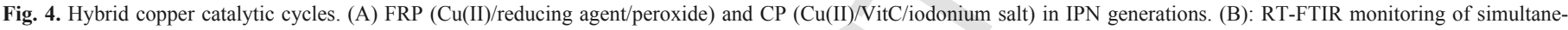

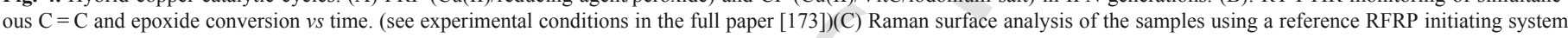

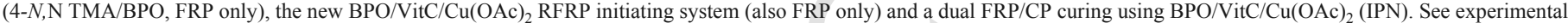
conditions in the original study [173]. Copyright 2018.

\subsection{Redox emulsion polymerization}

Emulsion polymerization is of huge importance. In the domain of waterborne coatings only - a leading product derived mainly from an emulsion polymerization - the market size was estimated in 2015 at 17.7 million tons worth EUR 52.4 billions [5]. Many challenges and opportunities are identified in emulsion polymers [273,274], among them, the reduction of the energy consumption during the synthesis is crucial $[5,154,275,276]$. This is the reason why many authors studied the initiation of emulsion/dispersion polymerization by redox systems $[277,278]$. Generally speaking, the goal of redox emulsion polymerization is to generate initiating species under mild conditions in the Interval I as described in Scheme 30. It has to be noticed that, in seeded systems, there is no Interval I. Generally speaking, a significant reduction of the energetic costs is expected by redox initiations.

In the literature, Asua et al. performed the emulsion polymerization of acrylates thanks to the KPS/amine redox initiating systems at a temperature of $40^{\circ} \mathrm{C}$ and under $\mathrm{N}_{2}$ purge; they especially pointed out that much more energy consumption was necessary to reach a similar efficiency in a conventional thermal emulsion polymerization $\left(80^{\circ} \mathrm{C}\right)$ [280]. In recent efforts to produce bio-based emulsion polymers at room temperature and under air, poly(diisoamylitaconate-co-isoprene) (PDII) elastomer was fabricated from itaconic acid, isoamyl alcohol, and isoprene by the RFRP initiating system based on $\mathrm{Fe}(\mathrm{II}) /$ persulfate/sulfur-based reducing agent [281]. FRP in emulsion can also lead to the production of polymer particles with high swelling ratios that show excellent performances in water depollution mem- branes [282]. In some examples, surfactants can have a non-innocent role in redox emulsion polymerization, particularly when oxidizing agents are ions such as peroxodisulfates [283]. A significant consideration in redox emulsion polymerization is the phase (e.g.micelle/solvent/aqueous) of the reducing and oxidizing systems [284]. Lack of control over kinetics can be overcome by controlling the feeding rate of reducing agent. More generally, stoichiometry cannot be considered as the ideal setup for the highest radical generating rate in RFRP.

\subsection{Redox graft polymerization and block copolymerization}

Redox grafting has potential applications in membranes or in more exotic post-modified materials [285-291]. The synthesis of block copolymers via a redox polymerization process was reviewed by $\mathrm{Oz}$ turk et al. a decade ago [293,294]. Currently [294], these types of strategies - though not always clearly identified as a redox polymerization - are still very convenient [295]. Indeed, the redox grafting part can occur at RT when post polymerization (to induce block copolymerization) can be triggered by another stimulus (e.g. thermal for labile azo compounds) [296-298]. Graft polymerization of surfaces was also proposed via redox cationic (silver salt/Fe(0) surface) polymerization of activated epoxides (EPOX). The adhesive properties were competitive with two anaerobic (Loctite ${ }^{\circledR}$ ) acrylate products [192] showing the importance of developing RCP methods in the adhesive industry.

Next, still in redox surface functionalization, the work by Ma et al. [126] on the functionalization of graphene oxide sheets shows an 
Interval 1: : Nucleation of monomer-swollen micelles

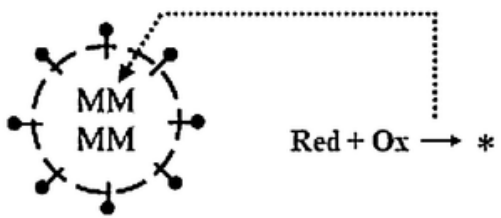

Interval II : Growth of latex particles

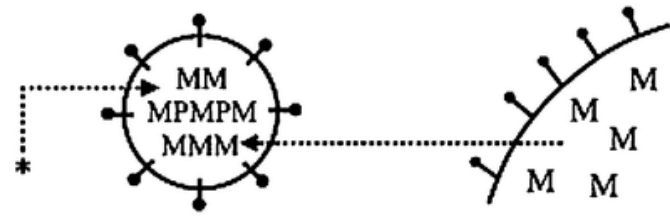

Latex particles

Monomer droplets

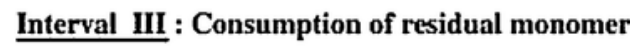

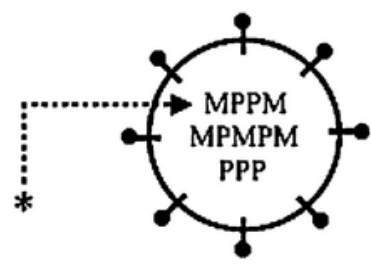

* Initiator radica

- Surlactant molecule

M Monomer molecule

P Polymer chain

Scheme 30. Schematic representation of the micelle nucleation model as proposed by Chern. In the case of redox emulsion polymerization, reducing (Red) and oxidizing (Ox) agents are used [279]. Copyright 2006. Adapted with permission from Elsevier Ltd.

interesting feature (Scheme 31): low grafting ratio leads to a conservation of the intercalated structure when too fast and high grafting leads to an exfoliated one. This exfoliation was also observed when an extremely fast photopolymerization of lamellar structures was performed [299]. Similarly, other users took advantage of Ce(IV) redox chemistry for the modification of carbon allotropic surfaces [300,301].

More complex architectures can be prepared under mild conditions using the redox polymerization [302]. For example, Huang et al.

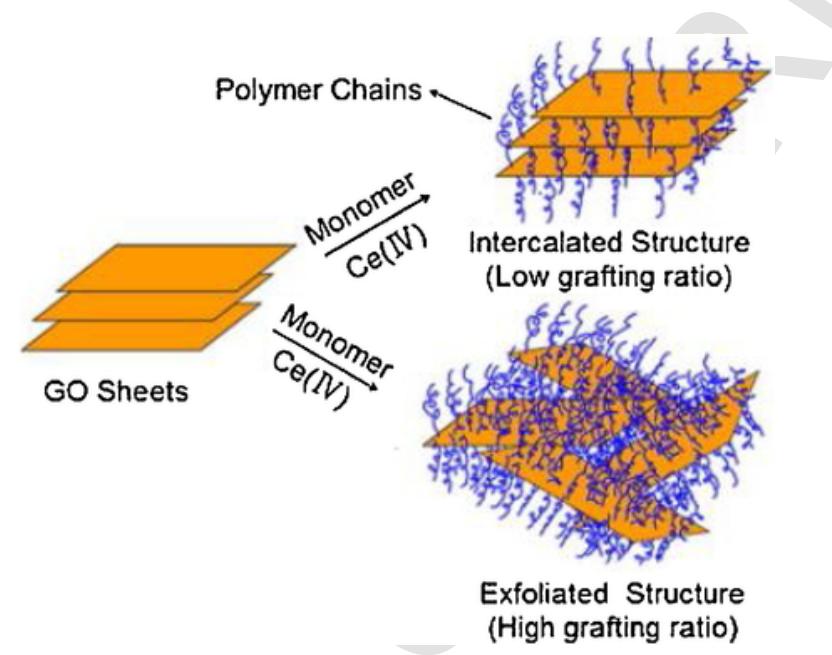

Scheme 31. Redox grafting from polymerization of graphene oxide sheets using a Ce(IV) redox system [126]. Copyright 2013. Adapted with permission from Elsevier Ltd. proposed to harvest the aminoalkyl radical produced by a persulfate/amine reaction (Scheme 32), doing so they were able to integrate vinylic polyethylene glycol, methacrylate and carboxylic acids moieties in hyperbranched plasticizers. Recently, polyoxazoline bearing acrylate moieties were grafted under redox conditions (persulfate $/ \mathrm{Fe}(\mathrm{II}) / \mathrm{NaHSO}_{3}$ at $35^{\circ} \mathrm{C}$ ) [303]. This result is of importance since polyoxazolines are water soluble polymers that are biodegradable and approved by the US Food and Drug Administration (FDA) [304,305].

\subsection{Redox synthesis of composites}

Aside from being an initiating technique requiring considerably low energy consumption, redox polymerization is extremely robust for composites synthesis. This is a considerable advantage compared to photopolymerization (also occurring at RT). RFRP or RCP initiating systems should have slow enough gel times and are embedded into resins containing fillers or fibers. Rheological considerations for the mixing are also to be taken into account.

Many redox based composites are available on the dental adhesives market [306]. Nevertheless, many times, the authors use the terms of auto-polymerization, chemically/self-cured resin cements instead of presenting the redox reaction that initiate the polymerization [307-309]. Other applications exist for these similar cements such as adhesives for metallic surfaces [310,311]. Very recently, as illustrated in Fig. 5, synthesis of RFRP dental composites was possible using amine-free peroxide-free respectively reducing and oxidizing agents stables in formulation (RT, under air) [312].

As RCP is less encountered, RCP for composites polymerization remains less studied. Potentially, RCP synthesis (at RT) of composites containing natural fibers is possible as the $\mathrm{CP}$ was already performed using natural fibers/EPOX thermal cationic initiation $\left(>100^{\circ} \mathrm{C}\right)$ [313]. The brilliant work by Sangermano et al. showed that RCP composites was possible through the EPOX polymerization using a $\mathrm{Cu}(\mathrm{II}) / \mathrm{VitC} /$ iodonium salt initiating system, the application was the consolidation of lime plaster [314].

\section{Conclusion}

Redox polymerization is an excellent way to lower energetic consumption associated with polymer synthesis. Indeed, mixing oxidizing and reducing agents leads to reduced activation energies for the polymerization initiation (e.g. $<80 \mathrm{~kJ} / \mathrm{mol}$ ) allowing the polymerization at mild temperatures (e.g. 0 to $45^{\circ} \mathrm{C}$ ) when it is necessary to heat the reaction medium in thermal polymerization initiations $(>120 \mathrm{~kJ} / \mathrm{mol}$ activation energies). Redox initiation was mainly applied in the literature to redox free radical polymerization (RFRP) or redox cationic polymerization (RCP). Several initiating systems have been proposed for FRP, the first category used peroxides (or other compounds bearing weak bonds) as oxidizing agents when Metal/reducing agent strategies are more and more proposed. For RCP initiation, onium salt decomposition is of outmost importance to generate superacids that will induce the polymerization of epoxide resins (and related monomers). One can also found redox agents (and strategies) in other fields of the polymer science for example controlled polymerization. Again, the reducing or oxidizing agents allow to: i) enhance polymerization kinetics and ii) work under milder conditions. Next, it is possible to propose dual-curing systems when combining redox initiation in the synthesis of interpenetrating polymer network (IPN) or in redox photoactived polymerization. Finally, several redox polymerization applications are present in the literature in adhesives, latex production, composites synthesis, biomaterial production, surface modification... Robustness of the systems under mild conditions 


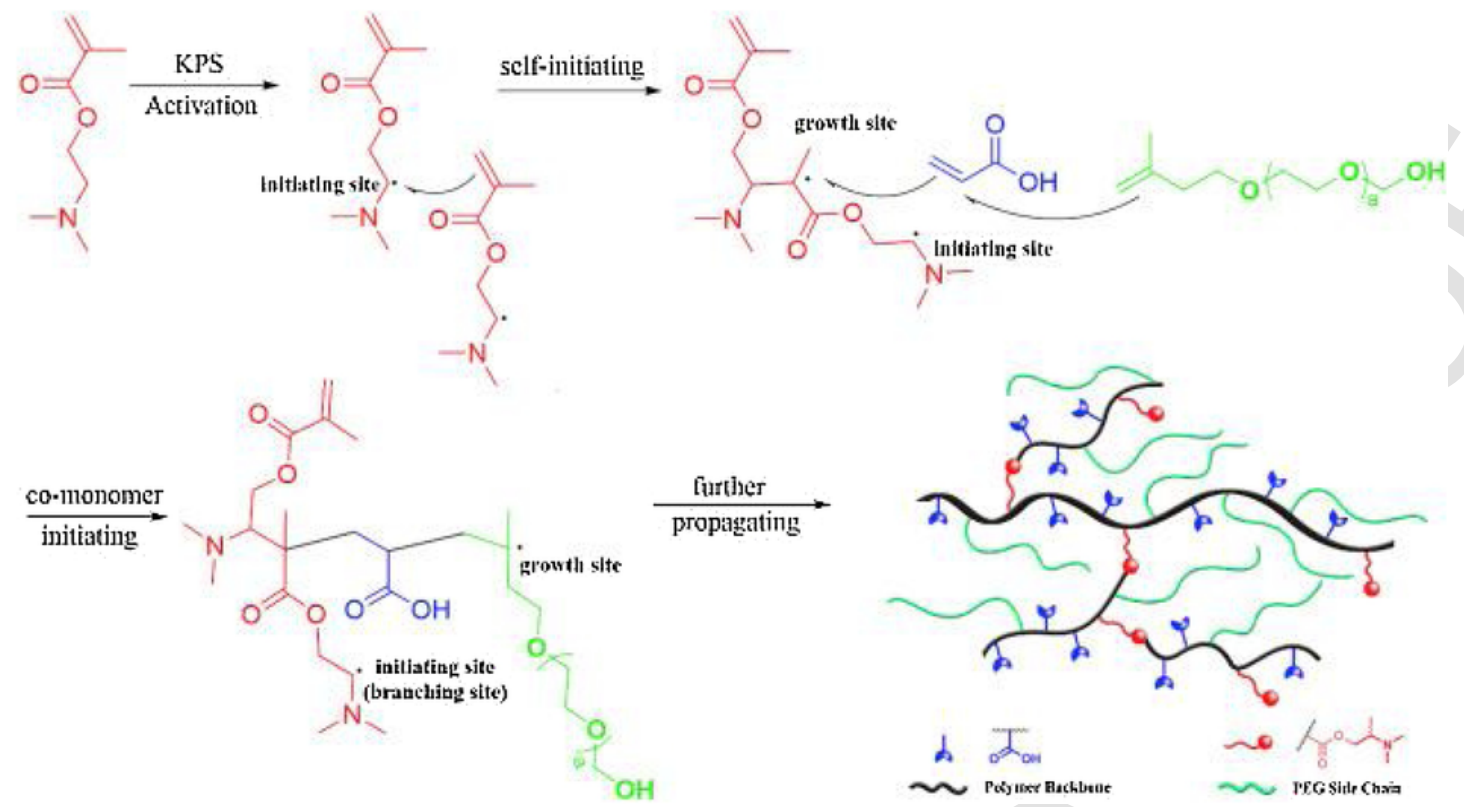

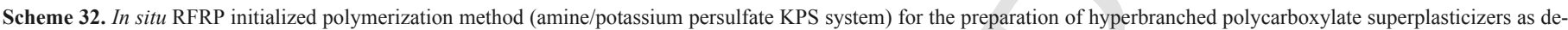
scribed by Huang et al. [302], Copyright 2013. Adapted with permission from Elsevier Ltd.
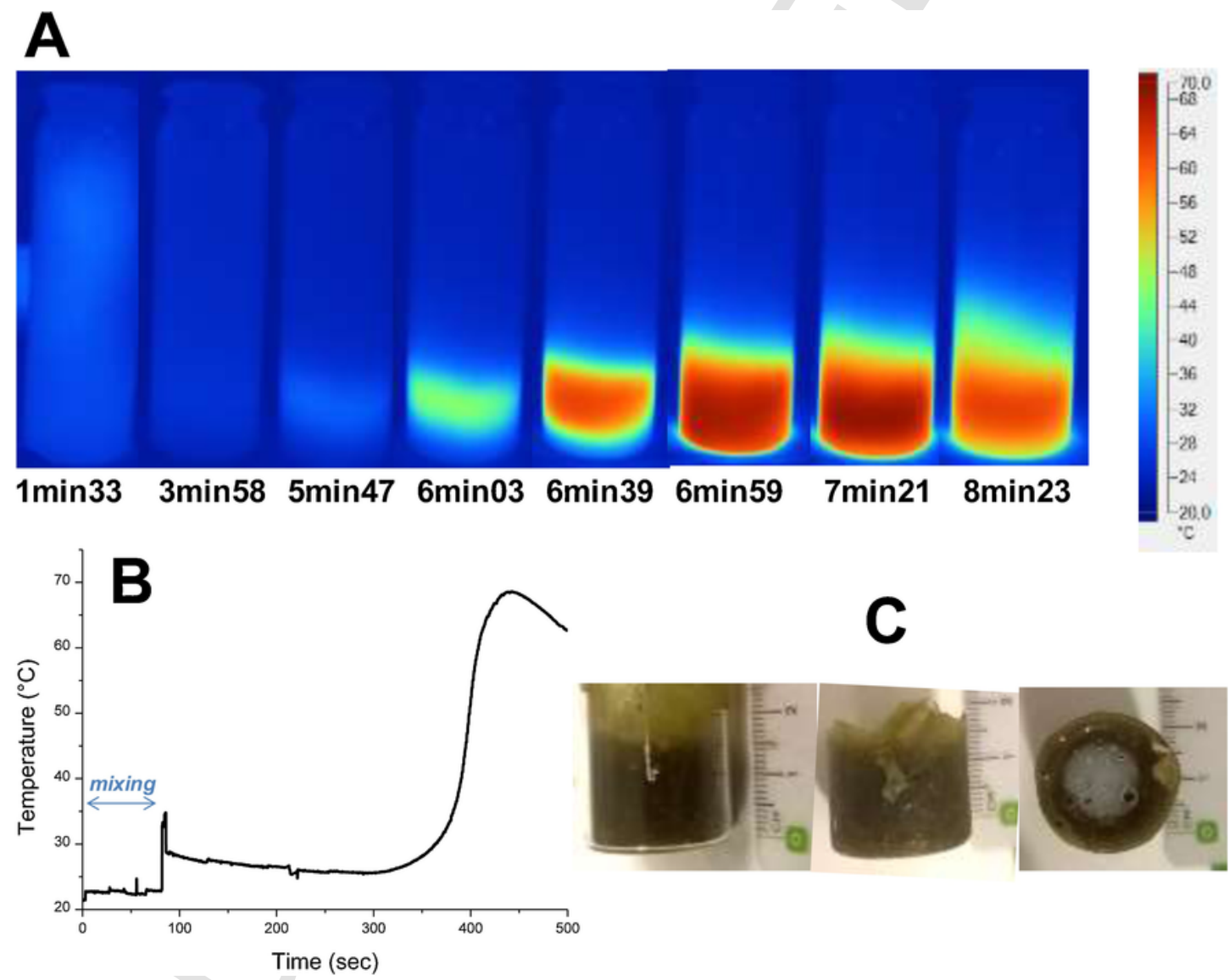

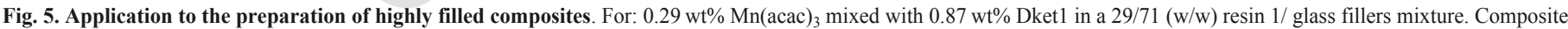

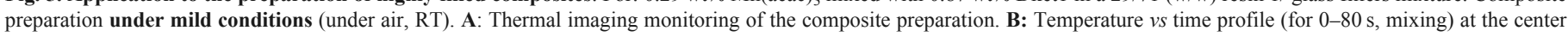

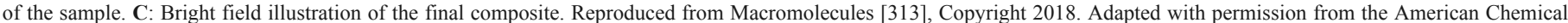
Society. 
ensures reduced energy consumptions. In the future, one can expect: i) the increasing presence of dual-curing systems involving redox reactions, ii) peroxide-free initiating systems, iii) harvesting the hydroperoxides formed through oxygen inhibition in one component (photo)redox systems robust in shadowed areas, iv) emergence of more RCP applications, v) rapid technology transfer in the composites, emulsion polymerization and surface grafting industries.

\section{Uncited references}

$[166,197,292]$.

\section{References}

[1] D. Braun, Origins and development of initiation of free radical polymerization processes, Int J Polym Sci 2010 (2009), e893234/1-10.

[2] A. Székely, M. Klussmann, Molecular radical chain initiators for ambient- to low-temperature applications, Chem Asian J 14 (2019) 105-115.

[3] 3] J. Lalevée, J.P. Fouassier, Dyes and chomophores in polymer science, John Wiley \& Sons Inc, New York, 2015, 435 pp.

[4] P. Garra, C. Dietlin, F. Morlet-Savary, F. Dumur, D. Gigmes, J.P. Fouassier, et al., Photopolymerization processes of thick films and in shadow areas: a review for the access to composites, Polym Chem 8 (2017) 7088-7101.

[5] F. Jasinski, P.B. Zetterlund, A.M. Braun, A. Chemtob, Photopolymerization in dispersed systems, Prog Polym Sci 84 (2018) 47-88.

[6] J.A. Pojman, Frontal polymerization, in: Matyjaszewski, M. Möller (Eds.), Polymer science: a comprehensive reference, Elsevier Ltd, Amsterdam, 2012, pp. 957-980.

[7] G.S. Misra, U.D.N. Bajpai, Redox polymerization, Prog Polym Sci 8 (1982) 61-131.

[8] A.S. Sarac, Redox polymerization, Prog Polym Sci 24 (1999) 1149-1204.

[9] P.J. Flory, The mechanism of vinyl Polymerizations1, J Am Chem Soc 59 (1937) 241-253.

[10] W.P. Hohenstein, H. Mark, Polymerization of olefins and diolefins in suspension and emulsion, Part II. J Polym Sci 1 (1946) 549-580.

[11] V.W. Kern, Die katalyse der polymerisationungesättigterverbindungenmithilfe von redoxsystemen1, Makromol Chem 1 (1948) 209-228.

[12] G.V. Schulz, Anregung von KettenpolymerisationendurchfreieRadikale II, Naturwissenschaften 27 (1939) 659-660.

[13] J.W. Breitenbach, A. Springer, K. Horeischy, Die stabilisierendeWirkung des Hydrochinons auf die Wärmepolymerisation des Styrols, Berichte Dtsch Chem Ges B Ser 71 (1) (1938) 438-441.

[14] R. Bacon, The initiation of polymerisation processes by redox catalysts, Q Rev ChemSoc 9 (1955) 287-310.

[15] R.G.R. Bacon, Reduction activation. A new polymerisation technique, Trans Faraday Soc 42 (1946) 140-155.

[16] A. Lagos, J. Reyes, Grafting onto chitosan. I: graft copolymerization of methy methacrylate onto chitosan with Fenton's reagent $(\mathrm{Fe} 2+/ \mathrm{H} 2 \mathrm{O} 2)$ as a redox initiator, J Polym Sci Part A: Polym Chem 26 (1988) 985-991.

[17] P. Garra, F. Dumur, M. Nechab, F. Morlet-Savary, C. Dietlin, B. Graff, et al., Stable copper acetylacetonate-based oxidizing agents in redox (NIR photoactivated) polymerization: an opportunity for the one pot grafting from approach and an example on a 3D printed object, Polym Chem 9 (2018) 2173-2182.

[18] A. Bhattacharya, Grafting: a versatile means to modify polymers. Techniques, factors and applications, Prog Polym Sci 29 (2004) 767-814.

[19] Y. Zhang, B. Gao, L. Gu, X. Zhao, Constructing aryl tertiary amine-bpo redox initiation system to realize highly effective graft-polymerization of styrene on silica gel particles, Acta Polym Sin (2012) 264-271.

[20] J. Furukawa, T. Tsuruta, S. Inoue, Triethylboron as an initiator for vinyl polymerization, J Polym Sci 26 (1957) 234-236.

[21] F.J. Welch, Polymerization of methyl methacrylate by triethylboron-oxygen mixtures, J Polym Sci 61 (1962) 243-252.

[22] A.A. Ermoshkin, D.C. Neckers, A.V. Fedorov, Photopolymerization without Light. Polymerization of Acrylates Using Oxalate Esters and Hydrogen Peroxide, Macromolecules 39 (2006) 5669-5674.

[23] J.V. Crivello, Redox initiated cationic polymerization, J Polym Sci Part A: Polym Chem 47 (2009) 1825-1835.

[24] S.C. Ligon, B. Husár, H. Wutzel, R. Holman, R. Liska, Strategies to reduce oxygen inhibition in photoinduced polymerization, Chem Rev 114 (2014) $557-589$.

[25] J. Yeow, R. Chapman, A.J. Gormley, C. Boyer, Up in the air: oxygen tolerance in controlled/living radical polymerisation, Chem Soc Rev 47 (2018) $4357-4387$
[26] N.A. Dotson, R. Galvan, R.L. Laurence, M. Tirrell, Polymerization process modeling, John Wiley \& Sons Inc, New York, 1995, 392 pp.

[27] P.R. Dvornić, M.S. Jaćović, The viscosity effect on autoacceleration of the rate of free radical polymerization, Polym Eng Sci 21 (1981) 792-796.

[28] P. Garra, B. Graff, F. Morlet-Savary, C. Dietlin, J.-M. Becht, J.-P. Fouassier, et al., Charge transfer complexes as pan-scaled photoinitiating systems: from $50 \mu \mathrm{m}$ 3D printed polymers at $405 \mathrm{~nm}$ to extremely deep photopolymerization (31 cm), Macromolecules 51 (2018) 57-70.

[29] George Odian, Principles of polymerization, 4th ed, John Wiley \& Sons Inc, New York, 2004, 832 pp.

[30] T.G. McKenzie, E. Colombo, Q. Fu, M. Ashokkumar, G.G. Qiao, Sono-RAFT polymerization in aqueous medium, Angew Chem Int Ed 56 (2017) $12302-12306$.

[31] Z. Wang, X. Pan, J. Yan, S. Dadashi-Silab, G. Xie, J. Zhang, et al., Temporal control in mechanically controlled atom transfer radical polymerization using low ppm of Cu catalyst, ACS Macro Lett 6 (2017) 546-549.

[32] J.P. Fouassier, J. Lalevée, Photoinitiators for polymer synthesis: Scope, reactivity, and efficiency, John Wiley \& Sons Inc, New York, 2012, 476 pp.

[33] Q. Yang, F. Dumur, F. Morlet-Savary, J. Poly, J. Lalevée, Photocatalyzed $\mathrm{Cu}$-Based ATRP involving an oxidative quenching mechanism under visible light, Macromolecules 48 (2015) 1972-1980.

[34] D.C. Neckers, W. Jager, Chemistry \& technology for UV \& EB formulation for coatings, inks \& paints, photoinitiation for polymerization: UV \& EB at the millenium, John Wiley \& Sons Inc, Chichester, 1999, 410 pp.

[35] M. Buback, A.M. van Herk, Radical polymerization: kinetics and mechanism, John Wiley \& Sons Inc, New York, 2007, 258 pp.

[36] W. Devonport, L. Michalak, E. Malmström, M. Mate, B. Kurdi, C.J. Hawker, et al., "Living" free-radical polymerizations in the absence of initiators: controlled autopolymerization., Macromolecules 30 (1997) 1929-1934.

[37] A.J. Clancy, M.K. Bayazit, S.A. Hodge, N.T. Skipper, C.A. Howard, M.S.P. Shaffer, Charged carbon nanomaterials: redox chemistries of fullerenes, carbon nanotubes, and graphenes, Chem Rev 118 (2018) 7363-7408.

[38] N. Casado, G. Hernández, H. Sardon, D. Mecerreyes, Current trends in redox polymers for energy and medicine, Prog Polym Sci 52 (2016) 107-135.

[39] A.K. O'Brien, C.N. Bowman, Impact of oxygen on photopolymerization kinetics and polymer structure, Macromolecules 39 (2006) 2501-2506.

[40] B. Husár, S.C. Ligon, H. Wutzel, H. Hoffmann, R. Liska, The formulator's guide to anti-oxygen inhibition additives, Prog Org Coat 77 (2014) $1789-1798$.

[41] Q. Yang, J. Lalevée, J. Poly, Development of a robust photocatalyzed ATRP mechanism exhibiting good tolerance to oxygen and inhibitors, Macromolecules 49 (2016) 7653-7666.

[42] M. Bouzrati-Zerelli, M. Maier, C.P. Fik, C. Dietlin, F. Morlet-Savary, J.P. Fouassier, et al., A low migration phosphine to overcome the oxygen inhibition in new high performance photoinitiating systems for photocurable dental type resins, Polym Int 66 (2017) 504-511.

[43] M. Schmitt, Method to analyse energy and intensity dependent photo-curing of acrylic esters in bulk, RSC Adv 5 (2015) 67284-67298.

[44] W.A. Braunecker, K. Matyjaszewski, Controlled/living radical polymerization: features, developments, and perspectives, Prog Polym Sci 32 (2007) 93-146.

[45] S. Shanmugam, J. Xu, C. Boyer, Light-regulated polymerization under Near-Infrared/Far-Red irradiation catalyzed by bacteriochlorophyll a, Angew Chem Int Ed 55 (2016) 1036-1040.

[46] D.M. Haddleton, C.B. Jasieczek, M.J. Hannon, A.J. Shooter, Atom Transfer Radical Polymerization of Methyl Methacrylate Initiated by Alkyl Bromide and 2-Pyridinecarbaldehyde Imine Copper(I) Complexes, Macromolecules 30 (1997) 2190-2193.

[47] M.A. Tasdelen, M. Çiftci, M. Uygun, Y. Yagci, Possibilities for photoinduced controlled radical polymerizations, ACS Symp Ser 1100 (2012) 59-72.

48] J.M. Nölle, C. Jüngst, A. Zumbusch, D. Wöll, Monitoring of viscosity changes during free radical polymerization using fluorescence lifetime measurements, Polym Chem 5 (2014) 2700-2703.

[49] P. Garra, F. Dumur, F. Morlet-Savary, C. Dietlin, J.P. Fouassier, J. Lalevée, A new highly efficient amine-free and peroxide-free redox system for free radical polymerization under air with possible light activation, Macromolecules 49 (2016) 6296-6309.

[50] N.J. Everall, Confocal Raman microscopy: common errors and artefacts, Analyst 135 (2010) 2512-2522.

[51] F. Courtecuisse, C. Dietlin, C. Croutxé-Barghorn, L.G.J. Van Der Ven, Depth characterization of photopolymerized films by confocal raman microscopy using an immersion objective, Appl Spectrosc 65 (2011) 1126-1132.

[52] P. Garra, F. Morlet-Savary, C. Dietlin, J.P. Fouassier, J. Lalevée, On-demand visible light activated Amine/Benzoyl peroxide redox initiating systems: a unique tool to overcome the shadow areas in photopolymerization processes, Macromolecules 49 (2016) 9371-9381. 
[53] B. Falk, S.M. Vallinas, J.V. Crivello, Monitoring photopolymerization reactions with optical pyrometry, J Polym Sci Part A: Polym Chem 41 (2003) 579-596.

[54] P. Garra, A.-H. Bonardi, A. Baralle, A. Al Mousawi, F. Bonardi, C. Dietlin, et al., Monitoring photopolymerization reactions through thermal imaging: A unique tool for the real-time follow- up of thick samples, 3D printing, and composites, J Polym Sci Part A: Polym Chem 56 (2018) 889-899.

[55] P. Garra, F. Dumur, A.A. Mousawi, B. Graff, D. Gigmes, F. Morlet-Savary, et al., Mechanosynthesized copper(I) complex based initiating systems for redox polymerization: towards upgraded oxidizing and reducing agents, Polym Chem 8 (2017) 5884-5896.

[56] P. Garra, F. Morlet-Savary, C. Dietlin, J.P. Fouassier, J. Lalevée, Charge-trans fer complexes as NewInhibitors/Photoinitiators for on-demand Amine/Peroxide redox polymerization, ACS Omega 3 (2018) 6827-6832.

[57] F. Lartigue-Peyrou, The use of phenolic compounds as free-radical polymerization inhibitors, Industrial Chemistry Library, Vol 8In: J.R. Desmurs, S. Ratton (Eds.), The roots of organic development, vol. 8, Elsevier Ltd, Amsterdam, 1996, pp. 489-505.

[58] J. Nicolas, Y. Guillaneuf, C. Lefay, D. Bertin, D. Gigmes, B. Charleux, Nitroxide-mediated polymerization, Prog Polym Sci 38 (2013) 63-235.

[59] D. Gigmes (Ed.), Nitroxide mediated polymerization: from fundamentals to applications in MaterialsScience, RSC Publishing, Cambridge, 2015, 512 pp.

[60] J. Szafko, K. Mańczyk, 4-Hydroxy-2,2,6,6-Tetramethylpiperidin-1-Oxyl as an inhibitor in the copolymerization of acrylonitrile with methyl methacrylate carried out in N,N- dimethylformamide, Makromol Chem Rapid Commun 1 (1980) 449-456.

[61] Liska R, Gorsche C, Seidler K, Moszner N. Sulfonic acid esters as regulators in radical polymerization reactions. US 20180142082A1, 2018

[62] H.J.H. Fenton, LXXIII.-Oxidation of tartaric acid in presence of iron, J Chem Soc Perkin Trans I 65 (1894) 899-910.

[63] P.L. Nayak, S. Lenka, Redox polymerization initiated by metal ions, J Macromol Sci Part C- Polym Rev 19 (1980) 83-134.

[64] P. Kalenda, J. Jarusek, Accelerating effects of ferrocene in polymerization reactions, Chem Pap 45 (1991) 119-125.

[65] I.I. Nasibullin, R.L. Safiullin, N.N. Sigaeva, L.R. Yakupova, S.V. Kolesov, Effect of metallocenes on benzoyl peroxide decomposition, React Kinet Catal Lett 56 (2015) 71-75.

[66] Y.B. Monakov, R.M. Islamova, A.K. Frizen, O.I. Golovochesova, S.V. Nazarova, Radical polymerization of methyl methacrylate in the presence of benzoyl peroxide, ferrocene and zirconocene dichloride, Mendeleev Commun 21 (2011) 206-208, Erratum 2012;22:55-55

[67] Y.I. Murinov, S.A. Grabovskiy, A.R. Kuramshina, A.V. Antipin, N.N. Kabal'nova,. The role of oxygen in the reaction of ferrocene with benzoyl peroxide, Russ J Gen Chem 85 (2015) 123-125.

[68] Y.I. Murinov, S.A. Grabovskiy, R.M. Islamova, A.R. Kuramshina, N.N. Kabal'nova, Mechanism of methyl methacrylate polymerization initiated by benzoyl peroxide and Ferrocene in the presence of oxygen, Mendeleev Commun 23 (2013) 53-55.

[69] P. Kalenda, Ferrocene and some of its derivatives used as accelerators of curing reactions in unsaturated polyester resins, Eur Polym J 31 (1995) 1099-1102.

[70] I.S. Voloshanovskii, O.V. Shevehenko, E.V. Burenkova, Benzoyl peroxide-cobalt(II) vinyl-beta- diketonate systems as initiators of styrene and methyl methacrylate polymerization., Russ J Appl Chem 81 (2008) 1033-1036.

[71] N. Kameda, E. Ishii, Polymerization of methyl methacrylate initiated by a Rh(I) complex and benzoyl peroxide, Makromol Chem 184 (1983) 1901-1906.

[72] J.K. Kochi, The decomposition of peroxides catalyzed by copper compounds and the oxidation of alkyl radicals by cupric salts, J Am Chem Soc 85 (1963) 1958-1968.

[73] K.L. Berry, J.H. Peterson, Tracer studies of oxidation—reduction polymerization and molecular weight of "Teflon" tetrafluoroethylene resin, J Am Chem Soc 73 (1951) 5195-5197.

[74] S.P. Manickam, U. Chandra Singh, K. Venkatarao, N.R. Subbaratnam, Peroxo salts as initiators of vinyl polymerization: 3 . Polymerization of methacrylic in acid initiated by perodisulphate ion- $\mathrm{pH}$ effect and metal ion catalysis, Polymer 20 (1979) 917-919.

[75] M. Worzakowska, Study of polymerization kinetics of the unsaturated polyester resin usingacetyl acetone peroxide and Co (II) octoate, J Therm Anal Calorim 88 (2007) 441-448.

[76] P. Garra, A. Kermagoret, A. Mousawi, F. Dumur, D. Gigmes, F. Morlet-Savary, et al., New copper (I) complex based initiating systems in redox polymerization and comparison with amine/benzoyl peroxide reference, Polym Chem 8 (2017) 4088-4097.

[77] N. Zivic, M. Bouzrati-Zerelli, A. Kermagoret, F. Dumur, J.P. Fouassier, D. Gigmes, et al., Photocatalysts in polymerization reactions, ChemCatChem 8 (2016) 1617-1631.
[78] P. Xiao, F. Dumur, J. Zhang, J.P. Fouassier, D. Gigmes, J. Lalevée, Copper complexes in radical photoinitiating systems: applications to free radical and cationic polymerization upon visible LEDs, Macromolecules 47 (2014) 3837-3844.

[79] P. Garra, F. Dumur, D. Gigmes, A. Al Mousawi, F. Morlet-Savary, C. Dietlin, et al., Copper (Photo)redox catalyst for radical photopolymerization in shadowed areas and access to thick and filled samples, Macromolecules 50 (2017) 3761-3771.

[80] L. Horner, K. Scherf, Über den einfluß der substituenten auf die chemischeReaktivität I. DerZerfall Von Dibenzoylperoxyddurchsubstituiertetertiäre Amine, Justus Liebigs Ann Chem 573 (1951) 35-55.

[81] J. Lal, R. Green, Effect of amine accelerators on the polymerization of methyl methacrylate with benzoyl peroxide, J Polym Sci 17 (1955) 403-409.

[82] H. Yang, L. Zhang, W. Ma, H. Pu, F. Gong, Polystyrene/Attapulgite nanocomposites prepared via in situ suspension polymerization with redox initiation system, J Appl Polym Sci 132 (2015), 41567/1-9.

[83] A. Zoller, D. Gigmes, Y. Guillaneuf, Simulation of radical polymerization of methyl methacrylate at room temperature using a tertiary amine/BPO initiating system, Polym Chem 6 (2015) 5719-5727.

[84] M. Worzakowska, The influence of tertiary aromatic amines on the BPO initiated cure of unsaturated epoxy polyesters with styrene studied by non-isothermal DSC, J Therm Anal Calorim 105 (2011) 987-994.

[85] T.-Y. Kwon, R. Bagheri, Y.K. Kim, K.-H. Kim, M.F. Burrow, Cure mechanisms in materials for use in esthetic dentistry, J Investig Clin Dent 3 (2012) $3-16$.

[86] I.D. Sideridou, D.S. Achilias, N.C. Kostidou, Copolymerization kinetics of dental dimethacrylate resins initiated by a benzoyl peroxide/amine redox system, J Appl Polym Sci 109 (2008) 515-524.

[87] I.D. Sideridou, D.S. Achilias, O. Karava, Reactivity of benzoyl peroxide/amine system as an initiator for the free radical polymerization of dental and orthopedic dimethacrylate monomers: effect of the amine and monomer chemical structure, Macromolecules 39 (2) (2006) 072-080.

[88] D.S. Achilias, I. Sideridou, Study of the effect of two BPO/amine initiation systems on the free- radical polymerization of MMA used in dental resins and bone cements, J Macromol Part A 39 (2002) 1435-1450.

[89] G.O. Wilson, J.W. Henderson, M.M. Caruso, B.J. Blaiszik, P.J. McIntire, N.R. Sottos, et al., Evaluation of peroxide initiators for radical polymerization-based self-healing applications, J Polym Sci Part A: Polym Chem 48 (2010) 2698-2708.

[90] M. Buback, H. Frauendorf, F. Günzler, P. Vana, Electrospray ionization mass spectrometric end- group analysis of PMMA produced by radical polymerization using diacyl peroxide initiators, Polymer 48 (2007) 5590-5598.

[91] K. Kim, N.R. Singstock, K. Childress, J. Sinha, A.M. Salazar, S.N. Whitfield, et al., Rational design of efficient amine reductant initiators for amine-peroxide redox polymerization, J Am Chem Soc 141 (2019) 6279-6291.

[92] A.A. Ermoshkin, E.S. Nikolaeva, D.C. Neckers, A.V. Fedorov, New tetraalkylborate initiators for remote polymerization, Macromolecules 41 (2008) 9063-9066.

[93] P.A. Giguère, D. Lamontagne, Polarographic determination of benzoyl peroxide and cumene hydroperoxide, Can J Chem 29 (1951) 54-59.

[94] L. Grossi, J. Lusztyk, K.U. Ingold, The benzoyloxy radical: attempted photochemical generation for kinetic studies and some relative rate constants, J Org Chem 50 (1985) 5882-5885.

[95] R. Baron, A. Darchen, D. Hauchard, Electrocatalytic reduction of tert-butyl hydroperoxide at iron electrodes, Electrochim Acta 49 (2004) 4841-4847.

[96] E. Rizzardo, D.H. Solomon, Acid-induced free-radical decomposition of hydroperoxides: polymerization of methyl methacrylate with tert-butyl hydroperoxide and sulfuric acid, J Macromol Part A 14 (1980) 33-50.

[97] G. Quint, E. Rizzardo, D.H. Solomon, T.H. Spurling, Acid-induced free-radical decomposition of hydroperoxides: quantal calculations, J Macromol Part A 15 (1981) 527-532.

[98] P. Beaunez, G. Helary, G. Sauvet, Role of N,N-dimethyl-para-toluidine and saccharin in the radical polymerization of methyl methacrylate initiated by a redox system. I. Cumene hydroperoxide/copper saccharinate, J Polym Sci Part A: Polym Chem 32 (1994) 1459-1469.

[99] J. Sun, Y. Li, K. Hasegawa, A study of self-accelerating decomposition temperature (SADT) using reaction calorimetry, J Loss Prev Process Ind 14 (2001) 331-336

[100] J.E. Guillet, R.G.W. Norrish, The photolysis of polymethylvinylketone - II. The preparation of graft polymers, Proc R Soc Lond A 233 (1955) 172-183.

[101] J.H. Merz, W.A. Waters, A.-electron-transfer reactions. The mechanism of oxidation of alcohols with Fenton's reagent, Discuss Faraday Soc 2 (1947) $179-188$.

[102] K.F. Wissbrun, The photolysis of polymethylvinyl ketone and polymethyl isopropenyl Ketone1, J Am Chem Soc 81 (1959) 58-62. 
[103] I.M. Kolthoff, A.I. Medalia, H.P. Raaen, The reaction between ferrous Iron and peroxides. IV. Reaction with potassium persulfate, J Am Chem Soc 73 (1951) 1733-1739.

[104] R.J. Orr, H.L. Williams, The efficiency of initiation by Cumyloxy and sulfoxy radicals in free radical polymerization, J Am Chem Soc 77 (1955) 3715-3720.

[105] N. Lavrov, I. Moskaleva, T. Lavrova, Polymerization of 2-Hydroxyethyl methacrylate, initiated ammonium persulfate ascorbic-acid in aqueous-organic media, Russ J Appl Chem 66 (1993) 2098-2101.

[106] C. Larpent, E. Bernard, J. Richard, S. Vaslin, Polymerization in Microemulsions with polymerizable cosurfactants: a route to highly functionalized nanoparticles, Macromolecules 30 (1997) 354-362.

[107] N. Lavrov, Molecular-weight of Poly-N-Vinylsuccinimide prepared from aqueous-solutions in the presence of ammonium persulfate ascorbic-acid initiating system, Russ J Appl Chem 67 (1994) 305-307.

[108] P. Wiecinska, T. Graule, M. Szafran, 1-Ascorbic acid as a new activator in fabrication of ceramics by techniques using in situ polymerization, J Eur Ceram Soc 34 (2014) 1581-1589.

[109] R. Bera, A. Dey, D. Chakrabarty, Synthesis, Characterization, and drug release study of acrylamide-co-itaconic acid based smart hydrogel, Polym Eng Sci 55 (2015) 113-122.

[110] H. Huang, J.Q. Xu, Z.C. Guo, Synthesis of conducting polyaniline using compound oxidant, Adv Mater Res 87-88 (2009) 300-305.

[111] M. Sivakumar, K.P. Rao, Synthesis, characterization, and in vitro release of ibuprofen from poly(MMA-HEMA) copolymeric core-shell hydrogel microspheres for biomedical applications, J Appl Polym Sci 83 (2002) 3045-3054.

[112] S. Pang, M.Z. Fiume, Final report on the safety assessment of Ammonium, Potassium, and Sodium Persulfate, Int J Toxicol 20 (Suppl 3) (2001) 7-21.

[113] Z. Liu, Y. Han, C. Zhou, M. Zhang, W. Li, H. Zhang, et al., Seeded emulsion polymerization of butyl acrylate using a redox initiator system: kinetics and mechanism, Ind Eng Chem Res 49 (2010) 7152-7158.

[114] M.L. Leza, I. Casinos, G.M. Guzman, Graft Copolymerization of 4-Vinylpyridine Onto Modified Cellulosic Fibers. the Ceric Ion Concentration Effect, J Macromol Part A 27 (1990) 413-421.

[115] G. Renders, G. Broze, R. Jérôme, P. Teyssié, Acrylonitrile polymerization by Ceric lon-primary alcohol redox systems in aqueous nitric acid, J Macromol Part A 16 (1981) 1399-1412.

[116] S.K. Saha, A.K. Chaudhuri, Effect of amines on the ceric ion-initiated polymerization of vinyl monomers. II. Polymerization of acrylonitrile by ceric ion in presence of various substituted amines, J Polym Sci A1 10 (1972) 797-808.

[117] W.C. Hsu, J.F. Kuo, C.Y. Chen, Aqueous polymerization of acrylamide initiated by cerium (IV)- ethylenediamine tetraacetic acid redox system, J Polym Sci Part A: Polym Chem 30 (1992) 2459-2466.

[118] W.C. Hsu, J.F. Kuo, C.Y. Chen, Aqueous polymerization of acrylamide initiated by cerium(IV)- amino acid chelating agent redox initiators, J Polym Sci Part A: Polym Chem 31 (2) (1993) 67-74.

[119] C. Özeroğlu, N. İpek, Penicillamine-cerium (IV) initiator system for synthesis of hydrogel containing lithium methacrylate ionic groups, Adv Polym Techno 37 (2018) 3305-3314.

[120] K.C. Gupta, K. Behari, Cerium (IV)-2-chloroethanol redox-pair initiated polymerization of acrylamide in aqueous medium, J Polym Sci Part A: Polym Chem 24 (1986) 767-775.

[121] A.S. Saraç, H. Basak, A.B. Soydan, A. Akar, Polymerization of acrylamide by electrolytically generated Ce(IV)-organic acid redox systems, Angew Makromol Chem 198 (1992) 191-198.

[122] S. Paulrajan, A. Gopalan, N.R. Subbaratnam, K. Venkatarao, Polymerization of $\mathrm{N}, \mathrm{N}^{\prime}$ - methylenebisacrylamide initiated by CEIV-thiourea redox system, Polymer 24 (1983) 906-908.

[123] C. Yagci, U. Yildiz, Redox polymerization of methyl methacrylate with allyl alcohol 1, 2- butoxylate-block-ethoxylate initiated by Ce (IV)/HNO3 redox system, Eur Polym J 41 (2005) 177-184.

[124] T. Demappa, Polymerization of acrylonitrile initiated by cerium (IV)-oxalic acid redox system: A kinetic study, J Appl Polym Sci 108 (2008) 1667-1674.

[125] H. Xu, H. Yang, S. Xue, J. Pan, Q. Ni, F. Gong, Preparation of polydimethylaminoethyl methacrylate grafted attapulgite via ceric ion-induced redox polymerization, J Appl Polym Sci 132 (2015), 42762/1-7.

[126] L. Ma, X. Yang, L. Gao, M. Lu, C. Guo, Y. Li, et al., Synthesis and characterization of polymer grafted graphene oxide sheets using a Ce(IV)/HNO3 redox system in an aqueous solution, Carbon 53 (2013) 269-276.

[127] A. Shaygan Nia, W.H. Binder, Graphene as initiator/catalyst in polymerization chemistry., Prog Polym Sci 67 (2017) 48-76.

[128] Z. Chen, R. Cao, S. Ye, Y. Ge, Y. Tu, X. Yang, Graphene oxide/poly (N-isopropylacrylamide) hybrid film-based near-infrared light-driven bilayer actuators with shape memory effect, Sens Actuators B Chem 255 (2018) 2971-2978.

[129] C. Li, H. Zhu, T. Hou, J. Vongsvivut, J.X. Dai, F. She, et al., Simultaneous polymerization and crosslinking for the synthesis of molecular-level graphene oxide-polyacryl amide-CeOx composites, Chem Eng J 263 (2015) 27-37.
[130] Q. Wan, D. Xu, L. Mao, Z. He, G. Zeng, Y. Shi, et al., Facile fabrication of AIE-Active fluorescent polymeric nanoparticles with ultra-low critical micelle concentration based on Ce(IV) redox polymerization for biological imaging applications, Macromol Rapid Commun 38 (2017), 1600752/1-7.

[131] B.B. Snider, Manganese(III)-Based Oxidative Free-Radical Cyclizations, Chem Rev 96 (1996) 339-364.

[132] Z. Zheng, W. Wang, Y. Zhou, Z. Zhang, X. Zhu, Manganese(III) acetylacetonate initiated RAFT polymerizations: an alternative and versatile RAFT initiator, Polym Chem 5 (2014) 37-42.

[133] E.G. Kastning, H. Naarmann, H. Reis, C. Berding, Metal chelates as polymerization initiators, Angew Chem Int Ed 4 (1965) 322-327.

[134] K. Endo, A. Yachi, Polymerization of Methyl Methacrylate with Mn(acac)3 in the Presence of Organic Halides. Possibility of Molecular Weight Control of Polymer, Polym J 34 (2002) 320-324.

[135] J.-R. Caille, A. Debuigne, R. Jérôme, Quinone transfer radical polymerization (QTRP) of styrene: catalysis by different metal complexes, J Polym Sci Part A: Polym Chem 43 (2005) 2723-2733.

[136] K. Endo, A. Yachi, Molecular-weight-controlled polymerization of styrene with $\mathrm{Mn}(\mathrm{acac})(3)$ in combination with organic halides, Polym Bull (Berl) 46 (2001) 363-369.

[137] E. Bouwman, R. Van Gorkum, A study of new manganese complexes as potential driers for alkyd paints, J Coat Technol Res 4 (2007) 491-503.

[138] N.A. Lavrov, Kinetic features of polymerization of 2-hydroxyethyl methacrylate, initiated by the system manganese tris(acetylacetonate)-acetic acid, Russ J Appl Chem 68 (1995) 922-924.

[139] P.L. Nayak, R.K. Samal, M.C. Nayak, Aqueous polymerization of acrylonitrile initiated by the Mn3+/citric acid redox system, Eur Polym J 14 (1978) 287-290.

[140] J. Lalevée, X. Allonas, J.P. Fouassier, New access to the peroxyl radicals +02 , Chem Phys Lett 445 (2007) 62-67.

[141] P. Garra, F. Morlet-Savary, B. Graff, F. Dumur, V. Monnier, C. Dietlin, et al., Metal Acetylacetonate-Bidentate Ligand Interaction (MABLI) as highly efficient free radical generating systems for polymer synthesis, Polym Chem 9 (2018) 1371-1378.

[142] P. Garra, F. Dumur, D. Gigmes, M. Nechab, F. Morlet-Savary, C. Dietlin, et al., Metal acetylacetonate-bidentate ligand interaction (MABLI) (Photo)activated polymerization: toward high performance amine-free, peroxide-free redox radical (Photo)initiating systems, Macromolecules 51 (2018) 2706-2715.

[143] A.M. Parambil, Y.M. Puttaiahgowda, P. Shankarappa, Copolymerization of $\mathrm{N}$-Vinyl pyrrolidone with methyl methacrylate by Ti (III)-DMG redox initiator, Turk J Chem 36 (2012) 397-409.

[144] Y. Sun, G. Zhai, CuSO 4-catalyzed self-initiated radical polymerization of 2-(N, N- dimethylamino) ethyl methacrylate as an intrinsically reducing inimer, Chin J Polym Sci 31 (2013) 1161-1172.

[145] C. Bamford, G. Eastmond, D. Whittle, Network formation III-influence of organometallic initiator on network structure, Polymer 10 (1969) 771-783.

[146] J.C. Lapairy, G. Process, Procede de polymerisation de monomeresvinyliques et/ou d'oligomerescomportant au moins un radical vinylique, EP 1409558 B1 2002.

[147] J.E. Klee, M. Maier, C.P. Fik, J. Lalevée, J.P. Fouassier, F. Morlet-Savary, et al., Dental composition, EP 3075372 A1 2016.

[148] E.F. Soares, A.R. Costa, A.B. Correr, S.A. Vedovello, M. Vedovello Filho, F.A. Ogliari, et al., Effect of composite containing an iodonium salt on the bond strength of brackets to bovine enamel, Braz Dent J 25 (2014) 237-240.

[149] R.J. DeVoe, M.R.V. Sahyun, E. Schmidt, N. Serpone, D.K. Sharma, Electron transfer sensitized photolysis of' onium salts, Can J Chem 66 (1988) 319-324.

[150] P. Garra, A. Baralle, B. Graff, G. Schrodj, C. Dietlin, J.P. Fouassier, et al., Radical cations in versatile high performance initiating systems for thermal, redox and photopolymerizations, Macromolecules 51 (2018) 8899-8911.

[151] M.A. Tehfe, S. Schweizer, A.C. Chany, C. Ysacco, J.L. Clément, D. Gigmes, et al., On the synthesis, characterization and reactivity of N-Heteroaryl-Boryl radicals, a new radical class based on five-membered ring ligands, Chem Eur J 20 (2014) 5054-5063.

[152] J. Broggi, M. Rollet, J.L. Clément, G. Canard, T. Terme, D. Gigmes, et al., Polymerization initiated by organic Electron donors, Angew Chem Int Ed 55 (2016) 5994-5999.

[153] A. Mortamet, R.A. Pethrick, Redox initiated free radical polymerization of 4-methylstyrene, J Appl Polym Sci 123 (2012) 1539-1547.

[154] N. Ballard, J.M. Asua, Radical polymerization of acrylic monomers: an overview, Prog Polym Sci 79 (2018) 40-60.

[155] R.K. Sadhir, M.R.M. Luck, Expanding monomers: Synthesis, characterization, and applications, $1^{\text {st }}$ ed, CRC Press, Boca Raton, 1992, 416 pp.

[156] D.U. Shah, P.J. Schubel, Evaluation of cure shrinkage measurement techniques for thermosetting resins, Polym Test 29 (2010) 629-639.

[157] R. Hirata, E. Clozza, M. Giannini, E. Farrokhmanesh, M. Janal, N. Tovar, et al., Shrinkage assessment of low shrinkage composites using micro-computed tomography, J Biomed Mater Res Part B Appl Biomater 103 (2015) 798-806. 
[158] A. Moeck, B. Roberto, V. Petry, R. Weder, D. Helsby, Shrinkage of UV oligomers and monomers, Rad Tech Proc (2014), 5 pp.

[159] J.P. Pascault, R.J. Williams, Epoxy polymers: new materials and innovations, John Wiley \& Sons Inc, New York, 2009, 384 pp.

[160] M. Lecompère, X. Allonas, D. Maréchal, A. Criqui, Versatility of pyrylium Salt/Vinyl ether initiating system for epoxide dual-cure polymerization: kick-starting effect of the coinitiator, Macromol Rapid Commun 38 (2017), 1600660/1-6.

[161] J.V. Crivello, The discovery and development of onium salt cationic photoinitiators, J Polym Sci Part A: Polym Chem 37 (2000) 4241-4254.

[162] Sangermano Marco, Razza Nicolò, J.V. Crivello, Cationic UV-Curing: Technology and Applications, Macromol Mater Eng 299 (2014) 775-793.

[163] J. Dektar, N. Hacker, Photochemistry of diaryliodonium salts, J Org Chem 55 (6) (1990) 39-47.

[164] N.P. Hacker, D.V. Leff, J.L. Dektar, The photochemistry of diphenyliodonium halides: evidence for reactions from solvent-separated and tight ion pairs, $\mathrm{J}$ Org Chem 56 (2) (1991) 280-282.

[165] S.P. Pappas (Ed.), Radiation curing: Science and technology, Springer Science \& Business Media, Berlin, 2013, 440 pp.

[166] R. Bongiovanni, M. Sangermano, Encyclopedia of polymer science and technology. UV-Curing science and technology, John Wiley \& Sons Inc, New York, 20141-20.

[167] Y. Mizuta, Y. Ito, The Pursuit of Rapid Curing in UV Cationic Polymerization, Rad Tech Proc (2006) 8.

[168] J.V. Crivello, K. Dietliker, Photoinitiators for free radical cationic \& anionic photopolymerisation, John Wiley \& Sons Inc, New York, 1999, 586 pp.

[169] U. Bulut, J.V. Crivello, Investigation of the reactivity of epoxide monomers in photoinitiated cationic polymerization, Macromolecules 38 (2005) 3584-3595.

[170] J.V. Crivello, J.H.W. Lam, Redox cationic polymerization: the diaryliodonium salt/ascorbate redox couple, J Polym Sci Part A: Polym Chem 19 (1981) $539-548$.

[171] J.V. Crivello, J.L. Lee, Redox initiators for cationic polymerization: The diaryliodonium Salt/Sn(II) redox couple, Makromol Chem 184 (1983) 463-473.

[172] P. Garra, M. Carré, F. Dumur, F. Morlet-Savary, C. Dietlin, D. Gigmes, et al. Copper-based (Photo)redox initiating systems as highly efficient systems for interpenetrating polymer network preparation, Macromolecules 51 (2018) 679-688.

[173] B.G. Gu, Synthesis, Crystal Structure, and Charcterization of Copper(II) Acetate Complex, Bull Korean Chem Soc 22 (2001) 113-116.

[174] P. Garra, F. Dumur, H. Mokbel, V. Monnier, F. Morlet-Savary, C. Dietlin, et al., New synthetic route to a highly efficient photoredox catalyst by mechanosynthesis, ACS Omega 3 (2018) 10938-10944.

[175] A.A. Mousawi, A. Kermagoret, D.-L. Versace, J. Toufaily, T. Hamieh, B. Graff, et al., Copper photoredox catalysts for polymerization upon near UV or visible light: structure/reactivity/efficiency relationships and use in LED projector 3D printing resins, Polym Chem 8 (2016) 568-580.

[176] A. Önen, Y. Yagci, Redox-initiated cationic polymerization: the pyridinium salt/ascorbate redox couple, Polymer 38 (1997) 1423-1425.

[177] A. Ledwith, Possibilities for promoting cationic polymerization by common sources of free radicals, Polymer 19 (1978) 1217-1219.

[178] I. Reetz, V. Bacak, Y. Yagci, Thermally induced radical promoted cationic polymerization using a novel $\mathrm{N}$-allyloxypyridinium salt, Macromol Chem Phys 198 (2003) 19-28.

[179] H. Baumann, H.-J. Timpe, PhotoinduzierteZersetzung von diaryliodoniumund TriarylsulfoniumsalzendurchBenzoinderivate und Benzilketale, Z Für Chem 24 (2010) 18-19.

[180] Y.Y. Durmaz, N. Moszner, Y. Yagci, Visible light initiated free radical promoted cationic polymerization using acylgermane based photoinitiator in the presence of onium salts, Macromolecules 41 (2008) 6714-6718.

[181] J. Lalevée, H. Mokbel, J.-P. Fouassier, Recent developments of versatile photoinitiating systems for cationic ring opening polymerization operating at any wavelengths and under low light intensity sources, Molecules 20 (2015) 7201-7221.

[182] J. Lalevée, J.P. Fouassier, Recent advances in sunlight induced polymerization : role of new photoinitiating systems based on the silyl radical chemistry, Polym Chem 2 (2011) 1107-1113.

[183] J. Lalevée, J.P. Fouassier, Photopolymerisation initiating systems, RSC Publishing, Cambridge, 2018, 606 pp

[184] C. Dursun, M. Degirmenci, Y. Yagci, S. Jockusch, N.J. Turro, Free radical promoted cationic polymerization by using bisacylphosphine oxide photoinitiators: substituent effect on the reactivity of phosphinoyl radicals, Polymer 44 (2003) 7389-7396.

[185] M. Degirmenci, Y. Hepuzer, Y. Yagci, One-step, one-pot photoinitiation of free radical and free radical promoted cationic polymerizations, J Appl Polym Sci 85 (2002) 2389-2395.
[186] F. Yilmaz, A. Sudo, T. Endo, Allyl sulfonium salt as a novel initiator for active cationic polymerization of epoxide by shooting with radicals species, J Polym Sci Part A: Polym Chem 48 (2010) 4178-4183.

[187] J.V. Crivello, J.L. Lee, Redox-initiated cationic polymerization: the diaryliodonium salt/benzoin redox couple, J Polym Sci Part A: Polym Chem 21 (2003) 1097-1110.

[188] J.V. Crivello, J.L. Lee, Recent advances in thermally and photochemically initiated cationic polymerization, Polym J 17 (1985) 73-83.

[189] J.V. Crivello, Redox initiated cationic polymerization: reduction of triarylsulfonium salts by silanes, Silicon 1 (2009) 111-124.

[190] M. Molleo, J.V. Crivello, Redox-initiated cationic polymerization: reduction of dialkylphenacylsulfonium salts by silanes, Macromolecules 42 (2009) 3982-3991.

[191] J.V. Crivello, J.L. Lee, Redox initiated cationic polymerization: Silane-N-aryl heteroaromatic onium salt redox couples, J Polym Sci Part A: Polym Chem 48 (2010) 4484-4495.

[192] D.J. Farrell, C. McArdle, M. Doherty, J.M. Kelly, Surface promoted redox cationic polymerization of epoxy monomers catalyzed by silver salts, J Polym Sci Part A: Polym Chem 50 (2012) 2957-2966.

[193] M.-A. Tehfe, R. Jamois, P. Cousin, S. Elkoun, M. Robert, In situ synthesis and characterization of Silver/Polymer nanocomposites by thermal cationic polymerization processes at room temperature: initiating systems based on organosilanes and starch nanocrystals, Langmuir 31 (2015) 4305-4313.

[194] J.V. Crivello, Redox initiated cationic polymerization: reduction of diaryliodonium salts by 9- BBN, J Polym Sci Part A: Polym Chem 47 (2009) 5639-5651.

[195] R. Souane, M.A. Tehfe, J. Lalevée, D. Gigmes, J.P. Fouassier, New Initiating Systems for Thermal Cationic Polymerization at Ambient Temperature with in situ Formation of $\mathrm{Ag}(0)$ Nanoparticles: A Silane/Silver Salt Combination, Macromol Chem Phys 211 (2010) 1441-1445.

[196] L.M. Broomfield, R.M. Sebastián, J. Marquet, R. Schönfeld, Ambient temperature polymerisation of oxiranes initiated by the novel $\mathrm{MSbF} 6 / \mathrm{H} 2 \mathrm{O}$ co-initiator system, Polymer 53 (5) (2012) 632-640.

[197] M.M. Green, H.A. Wittcoff, Organic chemistry principles and industrial practice, Angew Chem Int Ed 43 (2004) 6407-6408.

[198] J. Lyu, Y. Gao, Z. Zhang, U. Greiser, P. Polanowski, J.K. Jeszka, et al., Monte carlo simulations of atom transfer radical (Homo)polymerization of divinyl monomers: applicability of Flory- stockmayer theory, Macromolecules 51 (2018) 6673-6681

[199] T. Engels, Thermoset adhesives: epoxy resins, acrylates and polyurethanes, in: Q. Guo (Ed.), Thermosets, Woodhead Publishing, Cambridge, 2012, pp. 228-253.

[200] S. Shi, C. Croutxé-Barghorn, X. Allonas, Photoinitiating systems for cationic photopolymerization: ongoing push toward long wavelengths and low light intensities, Prog Polym Sci 65 (2017) 1-41.

[201] J.V. Crivello, R. Acosta Ortiz, Benzyl alcohols as accelerators in the photoinitiated cationic polymerization of epoxide monomers, J Polym Sci Part A: Polym Chem 40 (2002) 2298-2309.

[202] S. Penczek, P. Kubisa, Matyjaszewski K. Cationic Ring-Opening Polymerization: 2. Synthetic Applications, Adv Polym Sci 28-29 (1985) 1-317.

[203] K. Matyjaszewski, Advanced materials by atom transfer radical polymerization, Adv Mater 30 (2018), 1706441/1-22.

[204] J. Demarteau, A. Kermagoret, I. German, D. Cordella, K. Robeyns, J. De Winter, et al., Halomethyl- cobalt(bis-acetylacetonate) for the controlled synthesis of functional polymers, Chem Commun (Camb) 51 (2015) 14334-14337.

[205] Vatankhah, M. Varnosfaderani, A.N. Keith, Y. Cong, H. Liang, M. Rosenthal, et al., Chameleon-like elastomers with molecularly encoded strain-adaptive stiffening and coloration, Science 359 (2018) 1509-1513.

[206] E. Mehravar, A. Iturrospe, A. Arbe, J.R. Leiza, J.M. Asua, Acrylic-based composite latexes containing nano-sized liquid crystalline domains, Polymer 108 (2017) 288-300.

[207] B. Wenn, M. Conradi, A.D. Carreiras, D.M. Haddleton, T. Junkers, Photo-induced copper- mediated polymerization of methyl acrylate in continuous flow reactors, Polym Chem 5 (2014) 3053-3060.

[208] X. Pan, M.A. Tasdelen, J. Laun, T. Junkers, Y. Yagci, K. Matyjaszewski, Photomediated controlled radical polymerization, Prog Polym Sci 62 (2016) $73-125$.

[209] N. Corrigan, S. Shanmugam, J. Xu, C. Boyer, Photocatalysis in organic and polymer synthesis, Chem Soc Rev 45 (2016) 6165-6212.

[210] C.H. Lim, M.D. Ryan, B.G. McCarthy, J.C. Theriot, S.M. Sartor, N.H. Damrauer, et al., Intramolecular charge transfer and ion pairing in N,N-DiarylDihydrophenazinePhotoredox catalysts for efficient organocatalyzed atom transfer radical polymerization, J Am Chem Soc 139 (2017) 348-355.

[211] S. Dadashi-Silab, S. Doran, Y. Yagci, Photoinduced Electron transfer reactions for macromolecular syntheses, Chem Rev 116 (2016) 10212-10275. 
[212] P. Chmielarz, M. Fantin, S. Park, A.A. Isse, A. Gennaro, A.J.D. Magenau, et al., Electrochemically mediated atom transfer radical polymerization (eATRP), Top Vol Polym Chem 69 (2017) 47-78.

[213] M. Qi, Q. Dong, D. Wang, J.A. Byers, Electrochemically switchable ring-opening polymerization of lactide and cyclohexene oxide, J Am Chem Soc 140 (2018) 5686-5690.

[214] M.A. Tasdelen, B. Kiskan, Y. Yagci, Externally stimulated click reactions for macromolecular syntheses, Prog Polym Sci 52 (2016) 19-78.

[215] F. De Bon, M. Fantin, A.A. Isse, A. Gennaro, Electrochemically mediated ATRP in ionic liquids: controlled polymerization of methyl acrylate in [BMIm] [OTf], Polym Chem 9 (2018) 646-655.

[216] Y. Wang, M. Fantin, S. Park, E. Gottlieb, L. Fu, K. Matyjaszewski, Electrochemically mediated reversible addition-Fragmentation chain-transfer polymerization, Macromolecules 50 (2017) 7872-7879.

[217] A.J.D. Magenau, N.C. Strandwitz, A. Gennaro, K. Matyjaszewski, Electrochemically mediated atom transfer radical polymerization, Science 332 (2011) 81-84.

[218] H. Mohapatra, M. Kleiman, A.P. Esser-Kahn, Mechanically controlled radical polymerization initiated by ultrasound, Nat Chem 9 (2016) 135-139.

[219] Z. Wang, Z. Wang, X. Pan, L. Fu, S. Lathwal, M. Olszewski, et al., Ultrasonication-induced aqueous atom transfer radical polymerization, ACS Macro Let 7 (2018) 275-280

[220] C. Barner-Kowollik, T.P. Davis, J. Heuts, M.H. Stenzel, P. Vana, M. Whittaker, RAFTing down under: tales of missing radicals, fancy architectures, and mysterious holes, J Polym Sci Part A: Polym Chem 41 (2003) 365-375.

[221] M. Fantin, F. Lorandi, A. Gennaro, A. Isse, K. Matyjaszewski, Electron transfer reactions in atom transfer radical polymerization, Synthesis 49 (2017) 3311-3322.

[222] N.V. Tsarevsky, K. Matyjaszewski, "Green" atom transfer radical polymerization: from process design to preparation of well-defined environmentally friendly polymeric materials, Chem Rev 107 (2007) 2270-2299.

[223] Y. Kwak, A.J.D. Magenau, K. Matyjaszewski, ARGET ATRP of methyl acrylate with inexpensive ligands and ppm concentrations of catalyst, Macromolecules 44 (2011) 811-819.

[224] A. Simakova, S.E. Averick, D. Konkolewicz, K. Matyjaszewski, Aqueous ARGET ATRP, Macromolecules 45 (2012) 6371-6379.

[225] J. Yeow, R. Chapman, J. Xu, C. Boyer, Oxygen tolerant photopolymerization for ultralow volumes, Polym Chem 8 (2017) 5012-5022.

[226] S. Shanmugam, J. Xu, C. Boyer, Photoinduced oxygen reduction for dark polymerization, Macromolecules 50 (2017) 1832-1846.

[227] N. Corrigan, J. Xu, C. Boyer, A photoinitiation system for conventional and controlled radical polymerization at visible and NIR wavelengths, Macromolecules 49 (2016) 3274-3285.

[228] G. Ng, J. Yeow, J. Xu, C. Boyer, Application of oxygen tolerant PET-RAFT to polymerization- induced self-assembly, Polym Chem 8 (2017) 2841-2851.

[229] S. Dadashi-Silab, K. Matyjaszewski, Temporal control in atom transfer radical polymerization using zerovalent metals, Macromolecules 51 (2018) 4250-4258.

[230] D. Konkolewicz, Y. Wang, M. Zhong, P. Krys, A.A. Isse, A. Gennaro, et al., Reversible-Deactivation Radical Polymerization in the Presence of Metallic Copper. A Critical Assessment of the SARA ATRP and SET-LRP Mechanisms, Macromolecules 46 (2013) 8749-8772

[231] M.E. Levere, N.H. Nguyen, H.-J. Sun, V. Percec, Interrupted SET-LRP of methyl acrylate demonstrates $\mathrm{Cu}(0)$ colloidal particles as activating species, Polym Chem 4 (2013) 686-694.

[232] D. Konkolewicz, Y. Wang, P. Krys, M. Zhong, A.A. Isse, A. Gennaro, et al., SARA ATRP or SET-LRP. End of controversy?, Polym Chem 5 (2014) 4396-4417.

[233] S.R. Samanta, A. Anastasaki, C. Waldron, D.M. Haddleton, V. Percec, SET-LRP of methacrylates in fluorinated alcohols, Polym Chem 4 (2013) 5563-5569.

[234] R. Whitfield, A. Anastasaki, G.R. Jones, D.M. Haddleton, Cu(0)-RDRP of styrene: balancing initiator efficiency and dispersity, Polym Chem 9 (2018) 4395-4403.

[235] D.M. Haddleton, A.J. Clark, M.C. Crossman, D.J. Duncalf, A.M. Heming, S.R. Morsley, et al., Atom transfer radical polymerisation (ATRP) of methy methacrylate in the presence of radical inhibitors, ChemCommun Chem Commun (1997) 1173-1174.

[236] H. Dong, K. Matyjaszewski, ARGET ATRP of 2-(Dimethylamino)ethyl Methacrylate as an Intrinsic Reducing Agent, Macromolecules 41 (2008) 6868-6870

[237] W. Jakubowski, K. Min, K. Matyjaszewski, Activators regenerated by Electron transfer for atom transfer radical polymerization of styrene, Macromolecules 39 (2006) 39-45.

[238] K. Matyjaszewski, W. Jakubowski, K. Min, W. Tang, J. Huang, W.A. Braunecker, et al., Diminishing catalyst concentration in atom transfer radical polymerization with reducing agents, Proc Natl Acad Sci 103 (2006) 15309-15314.
[239] B.M. Peterson, V. Kottisch, M.J. Supej, B.P. Fors, On demand switching of polymerization mechanism and monomer selectivity with orthogonal stimuli, ACS Cent Sci 4 (2018) 1228-1234.

[240] N.A. Agareva, V.F. Ivanov, A.P. Aleksandrov, N.M. Bityurin, Smirnova LA Free-radical polymerization of methyl methacrylate in the presence of high ferrocene concentrations (English), Polym Sci Ser A Chem Phys 46 (2004) $105-113$.

[241] M.M.P. Grutters, C. Müller, D. Vogt, Highly selective cobalt-catalyzed hydrovinylation of styrene, J Am Chem Soc 128 (2006) 7414-7415.

[242] O. Konuray, X. Fernández-Francos, X. Ramis, À. Serra, State of the art in dual-curing acrylate systems, Polymers 10 (2018), 178/1-24

[243] M. Bazile, H.A. Nichols, J.A. Pojman, V. Volpert, Effect of orientation on thermoset frontal polymerization, J Polym Sci Part A: Polym Chem 40 (2002) 3504-3508.

[244] H. Yu, Y. Fang, L. Chen, S. Chen, Investigation of redox initiators for free radical frontal polymerization, Polym Int 58 (2009) 851-857.

[245] M. Retailleau, A. Ibrahim, X. Allonas, Dual-cure photochemical/thermal polymerization of acrylates: a photoassisted process at low light intensity, Polym Chem 5 (2014) 6503-6509.

[246] M. He, X. Huang, Y. Huang, Z. Zeng, J. Yang, Photoinduced redox initiation for fast polymerization of acrylaytes based on latent superbase and peroxides, Polymer 53 (2012) 3172-3177.

[247] M. Lecompère, X. Allonas, D. Maréchal, A. Criqui, Mechanistic approach to a photochemical/thermal dual-cure initiating system based on pyrylium salt-hydroperoxide for epoxide cationic polymerization, Polym Chem 8 (2017) 388-395.

[248] J.A. Pojman, G. Curtis, V.M. Ilyashenko, Frontal polymerization in solution, J Am Chem Soc 118 (1996) 3783-3784.

[249] M.F. Perry, V.A. Volpert, L.L. Lewis, H.A. Nichols, J.A. Pojman, Free-radical frontal copolymerization: the dependence of the front velocity on the monomer feed composition and reactivity ratios, Macromol Theory Simul 12 (2003) 276-286.

[250] K. Studer, C. Decker, E. Beek, R. Schwalm, N. Gruber, Redox and photoinitiated crosslinking polymerization I. Dual-cure isocyanate-acrylate system, Prog Org Coat 53 (2005) 126-133.

[251] M.A. Cole, K.C. Jankousky, C.N. Bowman, Redox initiation of bulk thiol-ene polymerizations, Polym Chem 4 (2013) 1167-1175.

[252] A.B. Lowe, Thiol-ene "click" reactions and recent applications in polymer and materials synthesis: a first update, Polym Chem 5 (2014) 4820-4870.

[253] P. Garra, F. Dumur, F. Morlet-Savary, C. Dietlin, D. Gigmes, J.P. Fouassier, et al., Mechanosynthesis of a Copper complex for redox initiating systems with a unique near infrared light activation, J Polym Sci Part A: Polym Chem 55 (2017) 3646-3655.

[254] H. Lu, A. Mehmood, A. Chow, J.M. Powers, Influence of polymerization mode on flexural properties of esthetic resin luting agents, J Prosthet Dent 94 (2005) 549-554

[255] M. Okuma, M. Nakajima, K. Hosaka, S. Itoh, M. Ikeda, R.M. Foxton, et al., Effect of composite post placement on bonding to root canal dentin using 1 -step self-etch dual-cure adhesive with chemical activation mode, Dent Mater J 29 (2010) 642-648.

[256] L.A. Jongsma, N. de Jager, C.J. Kleverlaan, P. Pallav, A.J. Feilzer, Shear bond strength of three dual- cured resin cements to dentin analyzed by finite element analysis, Dent Mater 28 (2012) 1080-1088.

[257] L. Feng, B.I. Suh, The effect of curing modes on polymerization contraction stress of a dual cured composite, J Biomed Mater Res Part B Appl Biomater 76 (2006) 196-202.

[258] X. Allonas, A. Ibrahim, V. Charlot, M. Retailleau, F. Karasu, C. Croutxé-Barghorn, Development of new photoinitiating systems for depth curing of thick materials, J Photopolym Sci Technol 28 (2015) 25-29.

[259] V. Kottisch, Q. Michaudel, B.P. Fors, Photocontrolled interconversion of cationic and radical polymerizations, J Am Chem Soc 139 (2017) 10665-10668.

[260] J. Demarteau, B. Améduri, V. Ladmiral, M.A. Mees, R. Hoogenboom, A. Debuigne, et al., Controlled synthesis of fluorinated copolymers via cobalt-mediated radical copolymerization of Perfluorohexylethylene and vinyl acetate, Macromolecules 50 (2017) 3750-3760.

[261] P.B.V. Scholten, J. Demarteau, S. Gennen, J. De Winter, B. Grignard, A. Debuigne, et al., Merging CO2-Based building blocks with cobalt-mediated radical polymerization for the synthesis of functional poly(vinyl alcohol)s, Macromolecules 51 (2018) 3379-3393.

[262] M.A. Tehfe, S. Elkoun, M. Robert, N-Vinylcarbazole: As an Additive for Thermal Polymerization at Room Temperature with in situ Formation of $\mathrm{Ag}(0)$ Nanoparticules, Appl Sci (Basel) 5 (2015) 241-258.

[263] K. Studer, C. Decker, C. Babe, E. Beck, C. Schwalm, N. Gruber, Redox and photoinitiated crosslinking polymerization II. Neat acrylate resin, Prog Org Coat 53 (2005) 134-146. 
[264] K. Studer, P.T. Nguyen, C. Decker, E. Beck, R. Schwalm, Redox and photoinitiated crosslinking polymerization - III. Clear and pigmented acrylic coatings, Prog Org Coat 54 (2005) 230-239.

[265] X.-L. Sun, H.-H. Sun, Y.-A. Wang, Polyacrylamide gel polymerization: ascorbic acid-ferrous sulfate-ammonium persulfate initiator for acid system, Acta BiochimBiophys Sin 30 (1998) 407-410.

[266] T. Billiet, M. Vandenhaute, J. Schelfhout, S. Van Vlierberghe, P. Dubruel, A review of trends and limitations in hydrogel-rapid prototyping for tissue engineering, Biomaterials 33 (2012) 6020-6041.

[267] E. Behravesh, S. Jo, K. Zygourakis, A.G. Mikos, Synthesis of in situ cross-linkable macroporous biodegradable poly (propylene fumarate-co-ethylene glycol) hydrogels, Biomacromolecules 3 (2002) 374-381.

[268] C. Özeroglu, A. Birdal, Swelling properties of acrylamide-N, N'-methylene bis (acrylamide) hydrogels synthesized by using meso-2, 3-dimercaptosuccinic acid-cerium (IV) redox couple, Express Polym Lett 3 (2009) 168-176.

[269] L. Sun, S. Zhang, J. Zhang, N. Wang, W. Liu, W. Wang, Fenton reaction-initiated formation of biocompatible injectable hydrogels for cell encapsulation, $\mathrm{J}$ Macromol Sci Part B- Phys 1 (2013) 3932-3939.

[270] J.L. Morán-Quiroz, E. Orozco-Guareño, et al., Polymeric hydrogels obtained using a redox initiator: application in $\mathrm{Cu}$ (II) ions removal from aqueous solutions, J Appl Polym Sci 131 (2014), 39933/1-11.

[271] K.H. Malinowska, T. Verdorfer, A. Meinhold, L.F. Milles, V. Funk, H.E. Gaub, et al., Redox-Initiated Hydrogel System for Detection and Real-Time Imaging of Cellulolytic Enzyme Activity, ChemSusChem 7 (2014) 2825-2831

[272] S. Shanmugam, J. Xu, C. Boyer, Aqueous RAFT photopolymerization with oxygen tolerance, Macromolecules 49 (2016) 9345-9357.

[273] D. Urban, K. Takamura (Eds.), Polymer dispersions and their industrial applications, Wiley-VCH Verlag $\mathrm{GmbH}$, Weinheim, 2002, 420 pp..

[274] J.M. Asua, Challenges and opportunities in continuous production of emulsion polymers: a review, Macromol React Eng 10 (2016) 311-323.

[275] J.M. Asua, Emulsion polymerization: from fundamental mechanisms to process developments, J Polym Sci Part A: Polym Chem 42 (2004) 1025-1041.

[276] S.H. Cho, J.H. Ryu, J.G. Park, K.D. Suh, Surface modification of monodisperse hydroxyl functionalized polymeric microspheres using ceric ammonium nitrate, Eur Polym J 41 (2005) 2209-2215.

[277] H. Warson, Redox polymerization in emulsion, ACS Symp Ser 24 (1976) 228-235.

[278] M. Errezma, A.B. Mabrouk, A. Magnin, A. Dufresne, S. Boufi, Surfactant-free emulsion Pickering polymerization stabilized by aldehyde-functionalized cellulose nanocrystals, Carbohydr Polym 202 (2018) 621-630.

[279] C.S. Chern, Emulsion polymerization mechanisms and kinetics, Prog Polym Sci 31 (443) (2006) 86.

[280] N. Kohut-Svelko, R. Pirri, J.M. Asua, J.R. Leiza, Redox initiator systems for emulsion polymerization of acrylates, J Polym Sci Part A: Polym Chem 47 (2009) 2917-2927.

[281] R. Wang, J. Ma, X. Zhou, Z. Wang, H. Kang, L. Zhang, et al., Design and preparation of a novel cross-linkable, high molecular weight, and bio-based elastomer by emulsion polymerization, Macromolecules 45 (2012) 6830-6839.

[282] S. Hernández, J.K. Papp, D. Bhattacharyya, Iron-based redox polymerization of acrylic acid for direct synthesis of Hydrogel/Membranes and metal nanopar ticles for water treatment, Ind Eng Chem Res 53 (2014) 1130-1142.

[283] S. Boutti, R.D. Zafra, C. Graillat, T.F. McKenna, Interaction of surfactant and initiator types in emulsion polymerisations: a comparison of ammonium persulfate and hydrogen peroxide, Macromol Chem Phys 206 (2005) 1355-1372.

[284] S. Wang, E.S. Daniels, E.D. Sudol, A. Klein, et al., Isothermal emulsion polymerization of n-butyl methacrylate with KPS and redox initiators: Kinetic study at different surfactant/initiator concentrations and reaction temperature, J Appl Polym Sci 133 (2016), 43037/1-8.

[285] B. Van der Bruggen, Chemical modification of polyethersulfone nanofiltration membranes: a review, J Appl Polym Sci 114 (2009) 630-642.

[286] E. Jin, N. Reddy, Z. Zhu, Y. Yang, Graft polymerization of native chicken feathers for thermoplastic applications, J Agric Food Chem 59 (2011) 1729-1738

[287] J.-H. Kim, P.-K. Park, C.-H. Lee, H.-H. Kwon, Surface modification of nanofiltration membranes to improve the removal of organic micro-pollutants (EDCs and PhACs) in drinking water treatment: graft polymerization and cross-linking followed by functional group substitution, J Membr Sci 321 (2008) 190-198.

[288] P. Suegama, H. De Melo, A.V. Benedetti, I. Aoki, Influence of cerium (IV) ions on the mechanism of organosilane polymerization and on the improvement of its barrier properties, Electrochim Acta 54 (2009) 2655-2662.

[289] A. Martínez-Hernández, A. Santiago-Valtierra, M. Alvarez-Ponce, Chemica modification of keratin biofibres by graft polymerisation of methyl methacrylate using redox initiation, Mater Res Innov 12 (2008) 184-191.
[290] A.L. Martinez-Hernandez, C. Velasco-Santos, M. de Icaza, V.M. Castaño, Grafting of methyl methacrylate onto natural keratin, E-Polym 3 (2003), 016/ $1-11$.

[291] L. Zhang, H. Yang, H. Liu, Q. Ni, F. Gong, Preparation and characterization of polystyrene-grafted attapulgite via surface-initiated redox polymerization, Polym Eng Sci 55 (2015) 889-895.

[292] T. Ozturk, I. Cakmak, Synthesis of block copolymers via redox polymerization process: a critical review, Iran Polym J 16 (2007) 561-581.

[293] F. Liu, Y. Wu, L. Bai, X. Peng, H. Zhang, Y. Zhang, et al., Facile preparation of hyperbranched glycopolymers via an AB3* inimer promoted by a hydroxy/ cerium(iv) redox process, Polym Chem 9 (2018) 5024-5031.

[294] E. Kot, A. Bismarck, Polyacrylamide containing weak temperature labile azo links in the polymer backbone, Macromolecules 43 (2010) 6469-6475.

[295] P. Lv, Y. Bin, Y. Li, R. Chen, X. Wang, B. Zhao, Studies on graft copolymerization of chitosan with acrylonitrile by the redox system, Polymer 50 (2009) 5675-5680.

[296] C.R. Haramagatti, S. Sikdar, S. Bhattacharya, Influence of concentration of redox couple on freeze-thaw stability of styrene-acrylic copolymer latex, J Coat Technol Res 13 (2016) 81-88.

[297] D. Gençoğlu, E.A. Güvel, G. Ülkü, N. Kızılcan, N. Köken, Copolymerization of Pyrrole and thienyl end capped poly(dimethylsiloxane) by Iron (III) chloride, World Conf Technol Innov Entrep 195 (2015) 2109-2116.

[298] M. Ashaduzzaman, M. Kunitake, Poly(methylmethacrylate)-block-poly(N- hydroxyethylacrylamide) diblock copolymers: direct ATRP synthesis and characterization, Iran Polym J 22 (2013) 493-499.

[299] C. Dol, F. Vibert, M.P. Bertrand, J. Lalevée, S. Gastaldi, E. Besson, Diazene-functionalized lamellar materials as nanobuilding blocks: application as light-sensitive fillers to initiate radical photopolymerizations, ACS Macro Lett 6 (2017) 117-120

[300] B. Wang, D. Yang, J.Z. Zhang, C. Xi, J. Hu, Stimuli-responsive polymer covalent functionalization of graphene oxide by $\mathrm{Ce}$ (IV)-induced redox polymerization, J Phys Chem C 115 (2011) 24636-24641.

[301] D. Yang, X. Zhang, C. Wang, Y. Tang, J. Li, J. Hu, Preparation of water-soluble multi-walled carbon nanotubes by $\mathrm{Ce}$ (IV)-induced redox radical polymerization, Prog Nat Sci 19 (2009) 991-996.

[302] Z. Huang, Y. Yang, Q. Ran, J. Liu, Preparing hyperbranched polycarboxylate superplasticizers possessing excellent viscosity-reducing performance through in situ redox initialized polymerization method, Cem Concr Compos 93 (2018) $323-330$.

[303] A.N. Blokhin, M.P. Kurlykin, A.B. Razina, M.M. Dudkina, A.V. Ten'kovtsev, Alkylsulfonyl Halides as initiators of cationic polymerization of Oxazolines, Polym Sci Ser B 60 (2018) 421-426.

[304] S. Kobayashi, Ethylenimine polymers, Prog Polym Sci 15 (1990) 751-823.

[305] M.C. Woodle, C.M. Engbers, S. Zalipsky, New amphipatic polymer-lipid conjugates forming long-circulating reticuloendothelial system-evading liposomes, [306] Bioconjug Chem 5 (1994) 493-496.

06] N. Moszner, U. Salz, Recent developments of new components for dental adhesives and composites, Macromol Mater Eng 292 (2007) 245-271.

[307] R.G. Fonseca, T.P. Artusi, J.G. dos Santos, G.L. Adabo, Diametral tensile strength of dual-curing resin cements submitted exclusively to autopolymerization, Quintessence Int 38 (797) (2007) e527-31.

[308] F. Reza, S.P. Lim, Effects of curing mode of resin cements on the bond strength of a titanium post: an intraradicular study, J Conserv Dent JCD 15 (2012) 123-126.

[309] J.E. McKenna, N.J. Ray, G. McKenna, F.M. Burke, The effect of variability in the Powder/Liquid ratio on the strength of zinc phosphate cement, Int J Dent 2011 (2011), 679315/1-4.

[310] I.A. Orsi, F.K. Varoli, C.H. Pieroni, M.C. Ferreira, E. Borie, In vitro tensile strength of luting cements on metallic substrate, Braz Dent J 25 (2014) $136-140$

[311] J.A. Soares, M. Brito-Júnior, D.R. Fonseca, A.F. Melo, S.M.C. Santos, Sotomayor NDCS, et al., Influence of luting agents on time required for cast post removal by ultrasound: an in vitro study, J Appl Oral Sci 17 (2009) 145-149.

[312] P. Garra, F. Dumur, M. Nechab, F. Morlet-Savary, C. Dietlin, B. Graff, et al., Peroxide-free and amine-free redox free radical polymerization: metal Acetylacetonates/Stable carbonyl compounds for highly efficient synthesis of composites, Macromolecules 51 (2018) 6395-6404

[313] K. Koschek, Design of natural fiber composites utilizing interfacial crystallinity and affinity, Compos Part Appl Sci Manuf 69 (2015) 21-29.

[314] A. Formia, J.M. Tulliani, P. Antonaci, M. Sangermano, Epoxy monomers consolidant for lime plaster cured via a redox activated cationic polymerization, J Cult Herit 15 (2014) 595-601 